\title{
Role of Neuroligins at the Inhibitory Postsynaptic Compartment of the Retina
}

\section{PhD Thesis}

\author{
in partial fulfillment of the requirements \\ for the degree "Doctor of Philosophy (PhD)" \\ in the Neuroscience Program \\ at the Georg August University Göttingen, \\ Faculty of Biology
}

Submitted by

Mrinalini Hoon

Born in

Kolkata, India

March 2010 


\section{Declaration}

I hereby declare that this thesis 'Role of Neuroligins at the Inhibitory Postsynaptic Compartment of the Retina' has been written independently, with no other aids than those cited.

Mrinalini Hoon 
"In the study of this membrane [the retina] I for the first time felt my faith in Darwinism (hypothesis of natural selection) weakened, being amazed and confounded by the supreme constructive ingenuity revealed not only in the retina and in the dioptric apparatus of the vertebrates but even in the meanest insect eye. ... I felt more profoundly than in any other subject of study the shuddering sensation of the unfathomable mystery of life."

— Santiago Ramón y Cajal 


\section{Table of Contents}

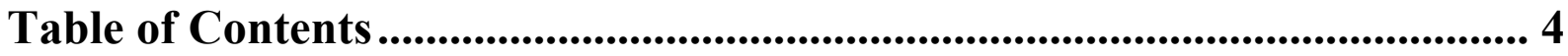

Table of Figures .......................................................................................................................... 7

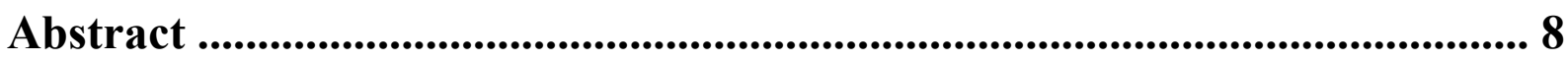

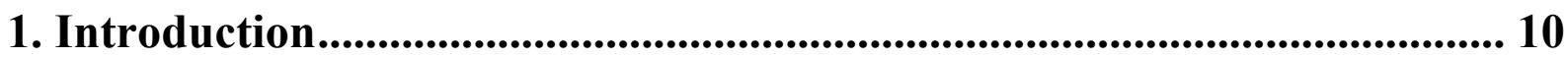

1.1 Neuroligins and synapse function ................................................................................. 10

1.1.1 Excitatory and inhibitory synaptic transmission ............................................... 10

1.1.2 Synaptogenesis and cell adhesion molecules ......................................................... 14

1.1.3 Characteristics and expression profile of Neuroligins............................................ 16

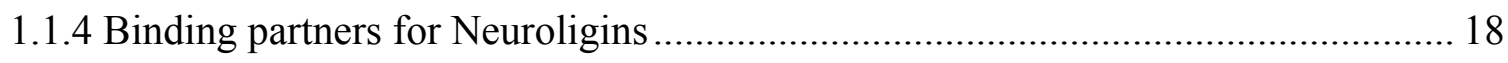

1.1.5 Neuroligins in synapse formation and function.................................................. 21

1.1.6 Neuroligins and cognitive function ................................................................. 22

1.2 The retina as a model system to explore synaptic function ......................................... 25

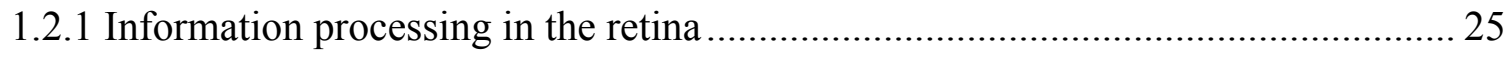

1.2.2 Synaptic architecture of the retina ..................................................................... 29

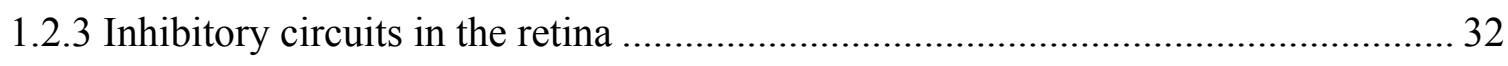

1.3 Aim of the study ...................................................................................................................... 35

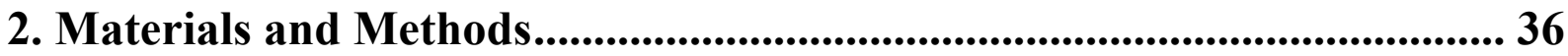

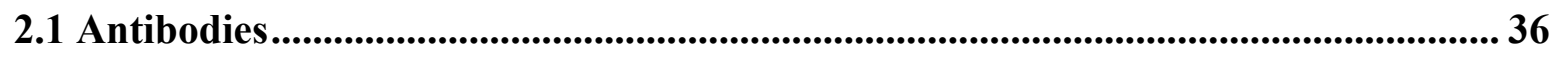

2.2 Immunofluorescence labeling in the retina .................................................................. 37

2.3 Electron Microscopy........................................................................................... 37

2.4 Imaging and Quantification.......................................................................................... 38

2.5 Immunohistochemistry and analysis of brain sections ................................................. 39

2.6 Multi-electrode array (MEA) recordings ...................................................................... 40

2.7 Patch clamp recordings from ganglion cells .................................................................. 40

2.8 Statistics............................................................................................................. 42

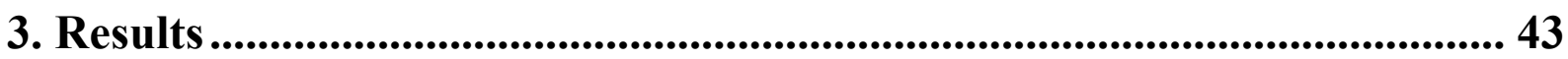

3.1 Distribution of Neuroligin isoforms in the mouse retina ............................................. 43

3.2 Functional characterization of Neuroligin 2 ........................................................... 45

3.2.1 Neuroligin 2 is preferentially localized to $\mathrm{GABA}_{\mathrm{A}}$ receptor containing synapses.. 45

3.2.2 Neuroligin 2 deficiency does not affect the core architecture of the retina.............. 49 
3.2.3 Lack of Neuroligin 2 results in a specific reduction of $\mathrm{GABA}_{\mathrm{A}}$ receptor clustering

3.2.4 Ganglion cells in the Neuroligin 2-deficient retina show increased baseline firing rate and impaired responses to light onset.

3.2.5 GABA receptor clustering is disrupted at the CA1 region of the hippocampus, subsequent to Neuroligin 2 deletion 56

3.3 Functional characterization of Neuroligin 4 ................................................................. 58

3.3.1 Neuroligin 4 is preferentially localized at glycinergic postsynapses. 58

3.3.2 Loss of Neuroligin 4 does not disrupt the synaptic network of the retina, but for a slight reduction in a GlyR subset.

3.3.3 Glycinergic mIPSCs from Neuroligin 4-deficient retinal ganglion cells exhibit a slower decay time constant. 63

3.3.4 Information processing in the Neuroligin 4-deficient retina 65

3.4 Morphological investigation of Neuroligin 3 ............................................................... 66

3.4.1 Neuroligin 3 is localized at GABAergic postsynapses of the retina ...................... 66

3.4.2 Retinal architecture is preserved in the absence of Neuroligin 3 68

3.4.3 Loss of Neuroligin 3 leads to a dramatic impairment in a $\mathrm{GABA}_{\mathrm{A}}$ receptor subset

3.5 Modulation of Neuroligin 4 expression in the retinae from Neuroligin 2-deficient mice 71

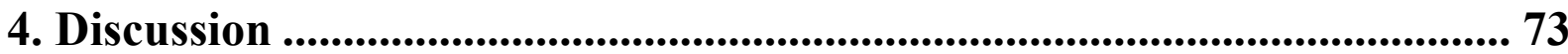

4.1 Distribution of Neuroligin isoforms in the retina ......................................................... 75

4.2 Postsynaptic architecture in the Neuroligin-deficient retina.................................... 78

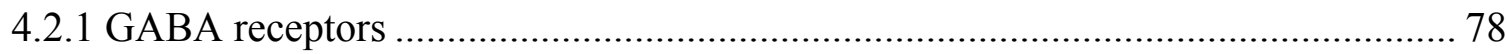

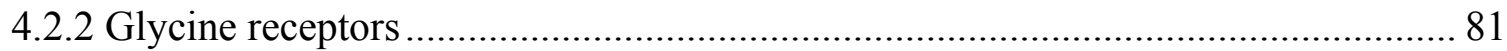

4.3 Retinal architecture in the absence of Neuroligin ............................................... 82

4.4 Information processing in the Neuroligin-deficient retina ..................................... 84

4.4.1 Information processing in the Neuroligin 2-deficient retina ................................ 84

4.4.2 Information processing in the Neuroligin 4-deficient retina ................................ 88

4.4.3 Functional consequences of Neuroligin 3 deletion............................................... 93

4.5 Neuroligins and sensory processing ….................................................................................... 96 
Summary ......................................................................................................................... 97

Acknowledgments ................................................................................................................. 98

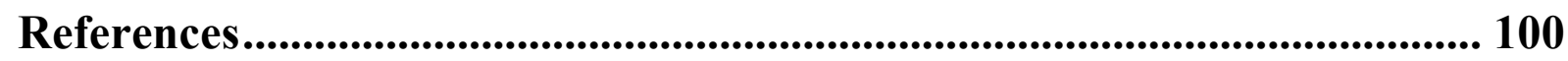

Curriculum Vitae ............................................................................... 112

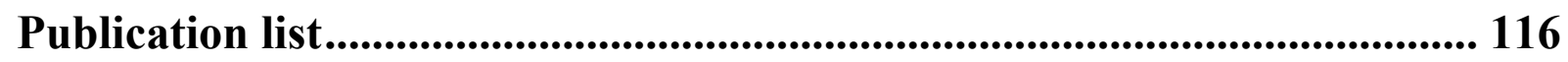




\section{Table of Figures}

Figure 1.1: Interactions of the Neuroligin-Neurexin link at the synapse.................................. 20

Figure 1.2: Information processing in the retina........................................................................ 28

Figure 1.3: Synaptic architecture of the retina ............................................................................ 31

Figure 3.1: Expression of Neuroligin isoforms in the mouse retina ................................................. 44

Figure 3.2: Neuroligin 2 is localized preferentially at GABAergic postsynapses of the retina $\ldots . . .47$

Figure 3.3: Association of Neuroligin 2 with different $\mathrm{GABA}_{\mathrm{A}}$ receptor subsets ........................ 48

Figure 3.4: Ultrastructural localization of Neuroligin 2 at symmetric synapses of the IPL........... 49

Figure 3.5: Organization of the Neuroligin 2-deficient retina....................................................51

Figure 3.6: Specific impairment of $\mathrm{GABA}_{\mathrm{A}}$ receptor clustering subsequent to Neuroligin 2 deletion

53

Figure 3.7: Light responses of Neuroligin 2-deficient ganglion cells ......................................55

Figure 3.8: Lamina specific loss of $\mathrm{GABA}_{\mathrm{A}}$ receptor clustering in the CA1 region of the Neuroligin

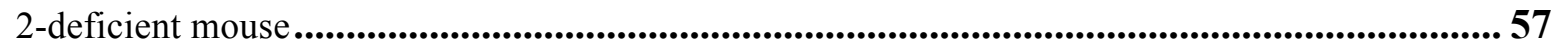

Figure 3.9: Neuroligin 4 is localized specifically at glycinergic postsynapses of the retina............ 60

Figure 3.10: Structural organization of the Neuroligin 4-deficient retina .................................. 61

Figure 3.11: Specific loss of $\alpha 1$ containing GlyR clusters in the Neuroligin 4-deficient retina $\ldots 62$

Figure 3.12: Single cell recordings from Neuroligin 4-deficient ganglion cells ............................ 64

Figure 3.13: Coding of light in the Neuroligin 4-deficient ganglion cells......................................65

Figure 3.14: Localization of Neuroligin 3 in the mouse retina....................................................6 67

Figure 3.15: Architecture of the Neuroligin 3-deficient retina.......................................................... 68

Figure 3.16: $\mathrm{GABA}_{\mathrm{A}} \alpha 2$ receptor clusters are specifically reduced in the absence of Neuroligin 3

Figure 3.17: GlyR subsets in the Neuroligin 3-deficient retina.................................................. 70

Figure 3.18: Upregulation of Neuroligin 4 in the Neuroligin 2-deficient retina ............................ 72 


\section{Abstract}

Neuroligins are postsynaptic cell adhesion proteins central to synapse maturation and function. There are four isoforms of Neuroligins (1-4) expressed in the rodent brain. Neuroligins 1 and 2 are localized at excitatory and inhibitory postsynapses, respectively. In contrast, the distribution and underlying function of the other two isoforms (Neuroligins 3 and 4) at central synapses have remained largely unresolved. Neuroligins are also expressed in humans, and interestingly, mutations in Neuroligins 3 and 4 have been found in a subset of autistic patients. The current study aims at characterizing the distribution and function of Neuroligins in the well-structured network of the mouse retina by using isoform-specific antibodies and single deletion mutant mice. The retina is well suited for both detailed anatomical analyses and functional assays, making it a robust system to study the role of Neuroligins in the formation and maintenance of a functional neural circuit and hence information processing.

The role of Neuroligin 2 in synaptic transmission and network function was first investigated. Throughout the retina, Neuroligin 2 is localized preferentially to inhibitory GABAergic postsynapses and largely precluded from glycinergic or glutamatergic postsynapses. Accordingly, the lack of Neuroligin 2 results in a severe reduction of $\mathrm{GABA}_{\mathrm{A}}$ receptor clustering at the inner synaptic layer, and in further subtle alterations of the retinal circuitry. Altogether, these impairments are detrimental to the coding of visual information by retinal ganglion cells, the output neurons of the retina. Taken together, these results indicate that Neuroligin 2 is essential for the functional integrity of GABAergic signaling and as a consequence, for information processing.

The next part of the study focused on the characterization of Neuroligin 4. Immunolabeling studies revealed the presence of Neuroligin 4 specifically at a subset of glycinergic postsynapses. The lack of Neuroligin 4 does not alter the retinal circuitry, yet causes a small reduction in the number of glycine receptor clusters containing the $\alpha 1$-subunit, known to confer fast kinetics to the receptors. As a result, glycinergic mIPSCs recorded from Neuroligin 4-deficient retinal ganglion cells exhibit slower decay kinetics. In summary, the absence of Neuroligin 4 leads to subtle deficits in the glycinergic inhibitory circuit at the inner synaptic layer of the retina.

The final phase of the study was devoted to Neuroligin 3. Neuroligin 3 also localizes selectively at inhibitory postsynapses, preferentially GABAergic, of the inner plexiform layer. Consequently, a loss of Neuroligin 3 leads to a dramatic reduction of a small subpopulation of 
$\mathrm{GABA}_{\mathrm{A}}$ receptor clusters, which may have important consequences for the efficacy of information processing by retinal ganglion cells.

In conclusion, this study yields the first characterization of three Neuroligin isoforms in the mouse retina and provides insights into their roles at the synapse and in visual information processing. Altogether, it demonstrates the importance of Neuroligins not only for the structural and functional integrity of large subsets of inhibitory synapses, but also for the maintenance of a network. 


\section{Introduction}

\subsection{Neuroligins and synapse function}

Normal brain function relies on the accurate formation, maturation and maintenance of connections between nerve cells. These connections form at specific sites of contact called synapses, which link roughly 10 billion neurons into functional networks capable of information processing and plasticity. Chemical synapses are asymmetric junctions where a presynaptic compartment containing neurotransmitter-filled vesicles is precisely aligned to a postsynaptic compartment bearing accumulations of neurotransmitter-specific receptors. There, an electrical signal is converted to a chemical message by the release of neurotransmitters, which upon binding to specific postsynaptic receptors, either facilitate or inhibit the activity of the postsynaptic neuron.

Functioning of synapses relies on their accurate formation and differentiation. This formation of synaptic contacts, called synaptogenesis, is a precisely regulated process in nervous system development, and primarily involves the interplay of cell adhesion molecules, scaffolding proteins, presynaptic vesicle machinery and postsynaptic neurotransmitter receptors.

Cell adhesion molecules ensure synapse assembly, maintenance and specificity by keeping pre- and postsynaptic compartments in register (Dityatev and El-Husseini, 2006). One such trans-synaptic heterophilic adhesion system is formed by the proteins Neurexins on the presynaptic membrane together with the Neuroligin proteins inserted on the postsynaptic side. The two proteins bind each other via their extracellular domains, and can also recruit additional (scaffolding) proteins via their intracellular domains, thus contributing to synapse organization and function (reviewed in Dean and Dresbach, 2006; Craig and Kang, 2007; Sudhof, 2008). Recent work indicates a crucial role of Neuroligins for the maturation and activity of synaptic contacts and also for higher cognitive function, as specific mutations in some human Neuroligin variants have been identified in a subset of autistic patients (reviewed in Sudhof, 2008; Bourgeron, 2009).

\subsubsection{Excitatory and inhibitory synaptic transmission}

A synapse is the junction where two neurons connect and communicate. Typically, the axon terminal of one neuron (called the presynaptic neuron) connects to the dendrite or soma (or even the axon) of its postsynaptic partner. At the site of contact, both the pre- and postsynaptic 
regions are characterized by specific structural and functional features, all of which aim to promote efficient synaptic transmission.

Synaptic transmission is initiated by an electrical signal called the action potential, which invades the presynaptic compartment. This leads to the opening of voltage-gated calcium channels enriched on the presynaptic membrane. The consequent influx of calcium ions initiates neurotransmitter release by a process called exocytosis. The latter occurs at a specialized membrane domain called the active zone of the presynaptic terminal (Couteaux and Pecot-Dechavassine, 1970; Landis, 1988). The dense cytomatrix of the presynaptic active zone is composed of several multidomain proteins, which interact with other synaptic proteins to organize the machinery of synaptic vesicle fusion and facilitate regulated and focal exocytosis (reviewed in Sudhof, 2004; Schoch and Gundelfinger, 2006). The neurotransmitter released from synaptic vesicles binds and activates clustered postsynaptic receptors, to mediate changes in membrane potential. These receptors are either ligand-gated ion channels (ionotropic), or G-protein coupled receptors (metabotropic) that activate intracellular signaling cascades. These receptors are mostly enriched at a specialized, electron-dense compartment called the postsynaptic density (PSD), which is composed of several scaffold proteins, cytoplasmic proteins, membrane-associated receptor clusters, and cell adhesion proteins such as Neuroligins (reviewed in Sheng and Hoogenraad, 2007).

In the brain, two antagonist neurotransmitter systems exist, that together account for the vast majority of synapses: an excitatory system that mainly employs the neurotransmitter glutamate, and an inhibitory system, which employs GABA and/or glycine as neurotransmitters. In the cortex, most neurons (about 80\%) are excitatory pyramidal cells, thus excitatory transmission mediates information transfer across the brain. The remaining neurons are inhibitory interneurons, which are organized into local circuits with their axons making short-range connections with target cells (McBain and Fisahn, 2001; Markram et al., 2004). Inhibitory transmission, thus, shapes the pattern of neuronal activity in networks and prevents them from hypersynchronous discharges (Lardi-Studler and Fritschy, 2007). Moreover, excitatory-inhibitory feedback loops between pyramidal cells and interneurons (Freund, 2003; Mann and Paulsen, 2007) facilitate the emergence of synchronous activity from populations of pyramidal neurons (Di Garbo, 2008). As an example, synchronous activity plays a crucial role in visual processing, for which several cortical areas work in parallel to code different features of an object (shape, orientation, colour, etc). The features associated to a particular object are then recombined, as assemblies of neurons coding for different features of sensory 
information fire in synchrony within a time scale of few milliseconds (Singer and Gray, 1995; Singer, 1999).

Excitatory and inhibitory synapses are segregated at different subcellular regions of the postsynaptic neuron. In cortical pyramidal cells or cerebellar Purkinje cells for example, excitatory synapses are formed at small dendritic protrusions called spines, whereas inhibitory synapses are located on the dendritic shaft, soma, or axon initial segment. Functionally, dendritic inhibition is suited to control the efficacy and plasticity of excitatory synapses onto pyramidal cells, while perisomatic inhibition controls the output of these cells (Freund, 2003; Mann and Paulsen, 2007).

Excitatory and inhibitory synapses also display distinct pre- and postsynaptic characteristics. On the presynaptic side, specific transporters mediate neurotransmitter uptake into synaptic vesicles at excitatory/glutamatergic (VGluT or vesicular glutamate transporters) (reviewed in Takamori, 2006) and inhibitory (a single identified transporter for GABA and glycine: VIAAT or vesicular inhibitory amino acid transporter) (Sagne et al., 1997; Wojcik et al., 2006) presynapses. Correspondingly, glutamate and GABA mediate their effects via a complement of distinct ionotropic (for fast responses) and metabotropic (for slower more sustained effects) receptors. Glycine, however, operates solely with an assembly of ionotropic receptors. The ultrastructural characteristics of the postsynaptic compartments further distinguish excitatory from inhibitory synapses, as excitatory postsynapses possess a broad and prominent PSD, and are termed asymmetric or type 1 synapses, while inhibitory postsynapses lack a prominent PSD, hence termed symmetric or type 2 synapses (Gray, 1959; Peters et al., 1991).

PSDs of excitatory synapses are enriched with two types of ionotropic glutamate receptors, namely NMDA (N-methyl-D-aspartic acid) and AMPA ( $\alpha$-amino-3-hydroxy-5-methyl-4isoxazolepropionic acid) receptors (reviewed in Okabe, 2007). These open upon glutamate binding to allow influx of cations, leading to postsynaptic depolarization (excitatory postsynaptic potential: EPSP) and hence signal propagation. Other essential constituents of the excitatory PSD, such as transmembrane AMPA regulatory proteins (TARPs) like Stargazin, have been shown to interact with AMPA receptors to regulate fast excitatory synaptic transmission (reviewed in Okabe, 2007). In addition, the excitatory PSD contains several scaffold proteins, among which PSD-95 is the best characterized. PSD-95 is the prototypical member of the MAGUK (membrane-associated guanylate kinase) family and is composed of three PDZ (PSD-95, Dlg and ZO-1 homology) domains followed by a src homology (SH3) and a guanylate kinase domain (reviewed in Kim and Sheng, 2004). The third PDZ domain of 
PSD-95 can interact with Neuroligins (Irie et al., 1997), while its first two PDZ domains facilitate interactions with NMDA receptors and voltage-gated potassium channels (Kim et al., 1995; Kornau et al., 1995). In addition, PSD-95 can regulate synaptic AMPA receptor concentration and activity by associating with the Stargazin-AMPA receptor complex (Chen et al., 2000). Thus, MAGUK proteins are key components of the excitatory PSD and play multiple roles in the organization and function of excitatory postsynapses.

At inhibitory postsynapses, fast effects are mediated by ionotropic GABA and glycine receptors, which are ligand-gated heteropentamers that form a chloride-permeable channel. In mature neurons, activation of these receptors results in the hyperpolarization of the postsynaptic membrane (inhibitory postsynaptic potential: IPSP). Ionotropic GABA receptors can be further differentiated into $\mathrm{GABA}_{\mathrm{A}}$ and $\mathrm{GABA}_{\mathrm{C}}$ receptors on the basis of their pharmacology (reviewed in Moss and Smart, 2001). GABA $A_{A}$ receptors are the prominent ionotropic GABA receptor population in the brain, composed of varied subunits ( $\operatorname{six} \alpha$, four $\beta$, three $\gamma$ and one $\delta$ ) (reviewed in Seeburg et al., 1990; Macdonald and Olsen, 1994; Sieghart, 1995) that confer to them immense structural and functional diversity. For example heterogeneity in the $\alpha$-subunit of $\mathrm{GABA}_{\mathrm{A}}$ receptors affects subcellular targeting, with the $\alpha 1$ subunit containing receptors being distributed equally at all inhibitory synapses on the soma, dendrites (proximal and distal) and the axon initial segment, while the $\alpha 2$-subunit containing $\mathrm{GABA}_{\mathrm{A}}$ receptors being localized preferentially at axo-axonic synapses on the axon initial

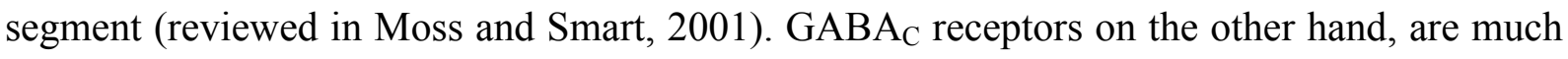
simpler in composition. They are homo/heteropentameric chloride channels composed of $\rho$ subunits $(\rho 1 / 2 / 3)$ (reviewed in Lukasiewicz, 1996) and their expression is restricted to the retinal synaptic layers (discussed in 1.2.3).

Inhibitory synapses lack a pronounced PSD, yet possess a core postsynaptic scaffold (Allison et al., 2000). In contrast to excitatory synapses however, PDZ domain-containing proteins are largely absent from inhibitory postsynapses demonstrating a principal difference in the molecular organization of excitatory and inhibitory PSDs (reviewed in Lardi-Studler and Fritschy, 2007). Gephyrin, a 93-kDa peripheral membrane protein, forms a scaffold and facilitates the postsynaptic localization and clustering of glycine receptors via a direct interaction (Meyer et al., 1995). In addition, Gephyrin can also bind signaling proteins like the GDP-GTP exchange factor (GEF) Collybistin, which is expressed only in the nervous system and exists as two splice variants (Reid et al., 1999; Kins et al., 2000). Importantly, coexpression of Collybistin II, Gephyrin and glycine receptor ( $\beta$-subunit) results in the 
translocation of Gephyrin and glycine receptors to the plasma membrane (reviewed in Moss and Smart, 2001). The heterogeneity, complexity and low-abundance of $\mathrm{GABA}_{\mathrm{A}}$ receptors, however, has made it difficult to identify proteins that govern their transport and postsynaptic clustering. However, one candidate protein suggested is Gephyrin, which is widely expressed in the central nervous system (CNS) including regions devoid of glycine receptors (Prior et al., 1992). Additionally, Gephyrin immunoreactivity colocalizes with $\mathrm{GABA}_{\mathrm{A}}$ subunits in different brain regions and deletion of Gephyrin leads to the reduction of $\mathrm{GABA}_{\mathrm{A}}$ receptor clusters in cultured neurons and brain sections (Kneussel et al., 1999). Yet, a direct interaction between Gephyrin and $\mathrm{GABA}_{\mathrm{A}}$ receptor subunits has not yet been shown. Further, apart from the gephyrin-dependent mechanism, gephyrin-independent mechanisms of postsynaptic $\mathrm{GABA}_{\mathrm{A}}$ receptor clustering have also been suggested, with Dystrophin being identified as a possible candidate for this process (reviewed in Moss and Smart, 2001; Fritschy et al., 2003). In summary, excitatory and inhibitory synapses bear distinct pre- and postsynaptic specializations that orchestrate excitatory and inhibitory transmission and hence network activity. The coordinated development of the specified pre- and postsynaptic compartments is thus crucial for nervous system development and occurs during the phase of "synaptogenesis".

\subsubsection{Synaptogenesis and cell adhesion molecules}

Synaptogenesis is the formation of synaptic contacts between neuronal processes, and involves the interplay of several secreted factors, receptors, and signaling molecules that enable neurons to form synapses. It requires interactions between specific adhesion molecules that mediate cell-cell recognition in the initial phase of synapse formation, as well as trigger the assembly, mechanical stabilization and differentiation of pre- and postsynaptic compartments. By homo- or heterophilic interactions, cell adhesion molecules hold the preand postsynaptic membranes in register after the initial contact between an axon terminal and a postsynaptic cell (reviewed in Waites et al., 2005). The intracellular domains of cell adhesion molecules are further coupled to signaling cascades allowing for the recruitment of scaffolding proteins and synaptic vesicles or neurotransmitter receptors, respectively, to the developing synapse. Cell adhesion molecules involved in synaptogenesis belong to diverse families. They include members of the Cadherin or immunoglobulin (Ig) superfamilies (for review, see Washbourne et al., 2004), as well as Neuroligins, Neurexins, SynCAMs and the LRRTM family of proteins (Scheiffele et al., 2000; Biederer et al., 2002; Graf et al., 2004; Linhoff et al., 2009; for review, see Yamagata et al., 2003; Washbourne et al., 2004; Craig et al., 2006; Dean and Dresbach, 2006). In particular, recent findings have indicated an 
important role of Neuroligins in the formation of synapses and their maturation (Scheiffele et al., 2000; Dean et al., 2003; Fu et al., 2003; Graf et al., 2004; Prange et al., 2004; Chih et al., 2005; Chubykin et al., 2005; Levinson et al., 2005; Varoqueaux et al., 2006; Dong et al., 2007).

Synaptogenesis is preceded by axonal path-finding whereby a developing growth cone (axon) is guided by a cohort of cell-surface and secreted cues (proteins) towards its presumptive target cell (reviewed in Goodman, 1996; Shen, 2004). This is followed by the initial contact formation between axons and dendrites during which cell adhesion molecules (CAMs) like members of the Cadherin superfamily are thought to position axons correctly in their target fields (reviewed in Waites et al., 2005). In the next phase, the newly formed contacts are stabilized by the accumulation of essential pre- and postsynaptic components. Preassembled transport packets deliver active zone components to presynaptic sites (Ahmari et al., 2000; Friedman et al., 2000). By electron microscopy, two large multi-domain active zone proteins, Bassoon and Piccolo, have been found to be associated with dense core vesicles (also called Piccolo/Bassoon transport vesicles) that ferry numerous components of the presynaptic active zone (Zhai et al., 2001). Presynaptic differentiation is then followed by differentiation of the postsynaptic compartment (Friedman et al., 2000; Okabe et al., 2001). Real-time imaging experiments have shown that postsynaptic components are recruited in a gradual manner, with no obvious involvement of transport vesicles (Bresler et al., 2004). The coordinated differentiation of the pre- and postsynaptic compartment is believed to involve trans-synaptic adhesion systems, which couple the recruitment of specific neurotransmitter release systems at the presynapse to distinct receptors at the postsynapse. Prospective candidates for this process include the Neurexin-Neuroligin heterophilic adhesion complex, and also the SynCAM mediated homophilic interactions (reviewed in Waites et al., 2005; Yamagata et al., 2003). Neuroligins can directly induce presynapse formation (Scheiffele et al., 2000). Furthermore, Neuroligins interact presynaptically with Neurexins, which have been shown to induce postsynaptic differentiation in dendrites (Graf et al., 2004). Thus, in vitro studies have shown that the Neurexin-Neuroligin link works as a bidirectional regulator of synapse initiation. However, recent in vivo analyses of the Neurexin ( $\alpha$-Neurexin knockout: Missler et al., 2003) and Neuroligin (Varoqueaux et al., 2006) knockout mice has shown that synapse structure and formation are not dramatically affected in these mutants, although synapse maturation and function is perturbed. Synapse maturation occurs once a nascent synapse has formed, and is a prolonged phase during which the pre- and postsynaptic compartments develop in a coordinated fashion, with the total number of synaptic vesicles and active zone area remaining 
proportional to the PSD area (Pierce and Mendell, 1993; Schikorski and Stevens, 1997). Finally, synaptic activity sculpts neuronal arbor growth and synapse formation (Wong and Wong, 2001; Knott et al., 2002; Hua and Smith, 2004), and activity-dependent pruning of synapses is critical for the ordered development of nervous system, for example in the formation of the ocular dominance columns in the visual cortex (Shatz and Stryker, 1978). Interestingly, deletion mutants lacking neurotransmitter release continue to exhibit normal synapse density (Verhage et al., 2000; Varoqueaux et al., 2002), indicating that activity may regulate synapse stability rather than synapse formation per se.

Interestingly, overexpression of Neuroligin 1 in hippocampal cultures was recently found to enhance the number of "functional" glutamatergic synapses, as blocking NMDA activity (required for the validation of excitatory synapses) impaired the ability of Neuroligin 1 to increase synapse number. Similarly, overexpression of Neuroligin 2 was found to act specifically on increasing inhibitory transmission, and blocking synaptic activity blocked the increase of functional inhibitory synapses mediated by Neuroligin 2 (Chubykin et al., 2007). These results provide in vitro evidence that overexpression of Neuroligins specifies/validates synaptic contacts in an activity dependent manner and is hence not responsible for the "initiation" of new synaptic contacts. Taking this in vitro data together with in vivo analysis of Neuroligin knockout mice, it seems likely that members of other adhesion protein families such as SynCAMs and Cadherins might mediate the initial contact between appropriate axons and dendrites, after which Neuroligins could act to mediate activity-dependent synapse maturation and recruitment of essential synaptic proteins (Chubykin et al., 2007; Craig and Kang, 2007).

In summary, the proper formation and function of synapses relies on the coordinated differentiation of the pre- and postsynaptic compartments. Diverse families of CAMs have been implicated in this process, among which are the Neuroligins that are ubiquitously present at developing and mature synapses of the CNS (Chih et al., 2005; Varoqueaux et al., 2006; for review, see Lise and El-Husseini, 2006; Craig and Kang, 2007).

\subsubsection{Characteristics and expression profile of Neuroligins}

Members of the Neuroligin family are type I transmembrane proteins, containing an extracellular N-terminal acetylcholinesterase homology domain, a glycosylated linker region that connects to the transmembrane domain, and a short C-terminal PDZ-binding domain (Figure 1.1). Neuroligins belong to the cholinesterase-like adhesion molecules (CLAMs), which include among others Neurotactin and Gliotactin (Gilbert and Auld, 2005). However, 
Neuroligins lack the complete catalytic triad in the active site of acetylcholinesterase, rendering the domain enzymatically inactive (Comoletti et al., 2003; Dean et al., 2003). Similar to acetylcholinesterase though, Neuroligins constitutively dimerize via their extracellular domain (Comoletti et al., 2003) and this dimerization of Neuroligins has been shown to be essential for a stable association (and/or signaling) with Neurexins (Dean et al., 2003).

There are five Neuroligin genes in humans (Jamain et al., 2003). In the rodent CNS, however, four isoforms (1-4) are expressed (Ichtchenko et al., 1995; Ichtchenko et al., 1996; Jamain et al., 2008), of which Neuroligin 1 and 2 have been studied most extensively. In humans, Neuroligins 3 and 4 are located at the X-chromosome, and Neuroligin 4 is complemented on the Y-chromosome as Neuroligin 4Y or Neuroligin 5 (reviewed in Sudhof, 2008). The members of the Neuroligin family show extensive homology with 52\% sequence identity, with their intracellular domains being less conserved than their extracellular regions (Ichtchenko et al., 1996). Further, all Neuroligins are alternatively spliced at a single conserved position (referred to as splice site A) besides Neuroligin 1, which is additionally spliced at a second (splice site B) position (Ichtchenko et al., 1996; Boucard et al., 2005).

The in situ hybridization analysis of Neuroligins 1-3 mRNA levels shows their ubiquitous expression throughout the newborn and adult rat brain (Varoqueaux et al., 2006). During the synaptogenic phase of brain development (postnatal day 0-24), all three Neuroligin mRNAs are concomitantly expressed and upregulated (Varoqueaux et al., 2006). Neuroligin 4 is also specifically expressed in the brain and upregulated in early postnatal stages until synapse formation is complete (Jamain et al., 2008).

Interestingly, specific Neuroligin isoforms have been shown to occupy specific synapse subtypes. Neuroligin 1 is found exclusively at excitatory (glutamatergic) postsynapses (Song et al., 1999), whereas Neuroligin 2 localizes selectively to inhibitory (GABA/glycinergic) postsynapses in the brain (Varoqueaux et al., 2004). Neuroligin 3, on the other hand, has been shown to be present at both GABA and glutamatergic synapses in hippocampal cultures (Budreck and Scheiffele, 2007); yet its distribution in vivo has not been addressed. Similarly, no study thus far has addressed the distribution and function of Neuroligin 4 at CNS synapses. In summary, Neuroligins are key adhesion proteins, which are upregulated during the development of the nervous system, and distinct Neuroligin isoforms have been characterized at specified synaptic subtypes. These findings served as a prime motivation for biochemical studies, which have started investigating the molecular interactions of the different Neuroligin isoforms at the postsynapse. 


\subsubsection{Binding partners for Neuroligins}

Neuroligins were initially discovered as endogenous ligands for presynaptically located Neurexins, and this interaction has been shown to be calcium-dependent (Ichtchenko et al., 1995). The mammalian genome contains three Neurexins genes, with two distinct promoters that drive the synthesis of a long $(\alpha)$-Neurexin and a shorter $(\beta)$-Neurexin variant (Tabuchi and Sudhof, 2002). Neurexins are also type I membrane proteins, with both Neurexin variants having identical transmembrane and carboxy terminal cytoplasmic domains but differing at their amino terminal extracellular regions. Extracellularly, $\alpha$-Neurexins have six LNS (Laminin, Neurexin, Sex-hormone-binding) domains (Rudenko et al., 1999), interspersed with three epidermal growth factor (EGF)-like domains; while $\beta$-Neurexins contain only the sixth LNS domain and lack the EGF-like sequences (Missler and Sudhof, 1998). In addition, Neurexins are subjected to extensive alternative splicing leading to a large repertoire of Neurexin isoforms (Ullrich et al., 1995).

The extracellular acetylcholinesterase domain of Neuroligins binds to the sixth LNS domain of Neurexins (both $\alpha$ - and $\beta$-). However, the binding affinities depend on alternative splicing of both Neurexins and Neuroligins (Chih et al., 2006; Comoletti et al., 2006). The splice site B of Neuroligin 1 regulates its binding to Neurexins. Neuroligins containing an insert (8 amino acid) at this position bind preferentially to $\beta$-Neurexins that lack an insert at their Splice site 4, whereas Neuroligins lacking an insert at splice site B can bind to both $\alpha$ - and $\beta$-Neurexins (regardless of splicing at position 4) (Boucard et al., 2005).

The cytoplasmic domains of both Neurexins and Neuroligins contain C-terminal PDZ-binding motifs, which enable multiple interactions with scaffolding proteins present at both pre- and postsynaptic compartments (Figure 1.1). At type 1 synapses, Neuroligins bind postsynaptic scaffold proteins like PSD-95 and S-SCAM, which in turn bind NMDA receptors and $\mathrm{K}^{+}$ channels to form extensive submembranous scaffolds containing several transmembrane and regulatory proteins (Cantallops and Cline, 2000). However, yeast two-hybrid experiments have shown that besides interacting with PSD-95 and S-SCAM, Neuroligin 1 can also associate with several other PDZ domain-containing proteins like SAP102, Chapsyn110, Magil and 3, Shank1 and 3, Pick1, GOPC, SPAR, Semcap3 and PDZ-RGS3 (Meyer et al., 2004). These results indicate that complex molecular interactions of Neuroligin 1 occur at the excitatory postsynapse, but a systematic analysis of Neuroligin 1 interactions with the different PDZ domain proteins has not yet been performed. 
As mentioned in 1.1.3, in vivo, Neuroligin 2 is localized at a majority of inhibitory postsynapses in the brain (Varoqueaux et al., 2004). However, the molecular associations of Neuroligin 2 at the inhibitory postsynapse (with GABA receptors or scaffolds such as Gephyrin) are only beginning to get unraveled. When this study began, the interaction partners of Neuroligin 2 were only speculated upon, and both gephyrin-dependent and gephyrinindependent mechanisms of Neuroligin 2 function at the inhibitory postsynapse were possible. The retina was therefore chosen as a model system, as in addition to glycinergic synapses that depend on Gephyrin for the postsynaptic localization and clustering of glycine receptors, the retina has distinct gephyrin-associated and gephyrin-independent $\mathrm{GABA}_{\mathrm{A}}$ receptor subsets (discussed in 1.2.3). Thus, it was the central aim of this project to study the distribution and function of Neuroligin 2 at both these $\mathrm{GABA}_{\mathrm{A}}$ receptor subsets in the retina. Additionally, Neuroligin 2 may interact directly with GABA and/or glycine receptors at the inhibitory postsynapse, but more work needs to be done to investigate these possible interactions.

As illustrated in Figure 1.1, the cytoplasmic tail of Neurexins also contains a PDZ-binding domain, through which Neurexins can interact with presynaptic scaffolding proteins to trigger the assembly of active zones (reviewed in Dean and Dresbach, 2006; Craig and Kang, 2007; Sudhof, 2008). For example, $\beta$-neurexins bind to $\mathrm{Ca}^{2+}$ Calmodulin-dependent serine protein kinase (CASK), which in turn forms a macromolecular protein complex that includes (N-type) voltage-gated $\mathrm{Ca}^{2+}$ channels (Butz et al., 1998; Maximov et al., 1999). Such interactions could lead to the stabilization of developing presynaptic terminals (Sankaranarayanan et al., 2003). Taken together, these in vitro-defined interactions have led to the hypothesis that the Neurexin-Neuroligin complex plays an essential role in maintaining the presynaptic release machinery and postsynaptic reception apparatus in register (Ichtchenko et al., 1996; Irie et al., 1997; Garner et al., 2002). 
A

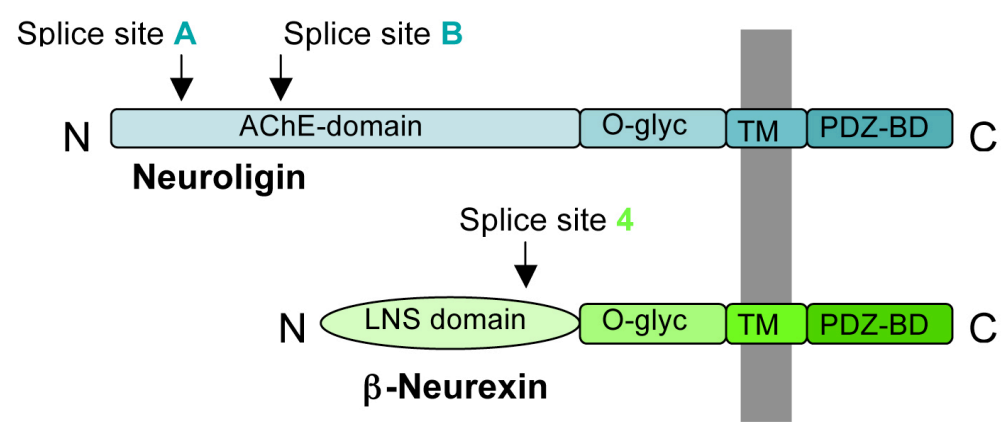

B

C
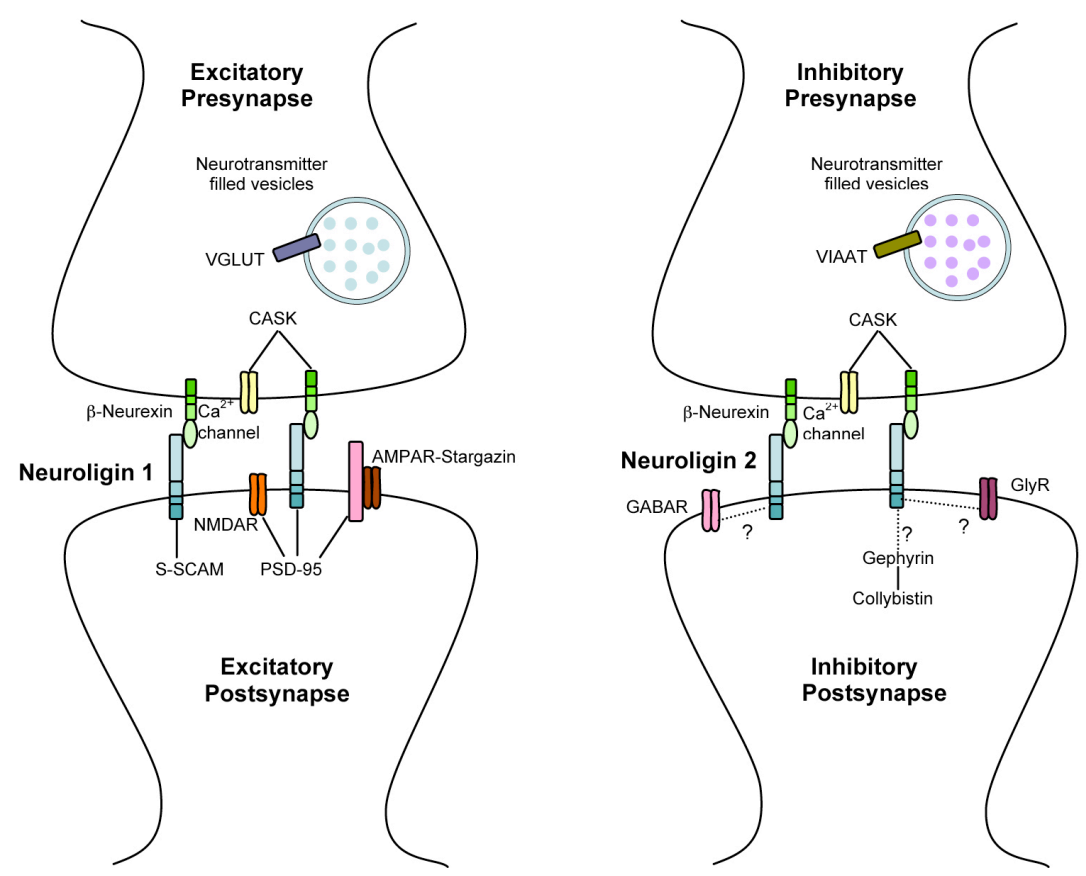

Figure 1.1: Interactions of the Neuroligin-Neurexin link at the synapse

Neuroligins interact with Neurexins via their acetylcholinesterase (AChE) homology domain, which binds to the LNS domain of Neurexins. Both proteins are connected via an O-glycosylation (O-glyc) domain to the transmembrane (TM) region, which is followed by a PDZ binding domain (PDZ-BD) (A). Neuroligin 1 is localized at excitatory postsynapses and interacts with PSD-95 to modulate glutamatergic transmission (B). Neuroligin 2, on the other hand, is localized at inhibitory postsynapses, and may interact directly with $\mathrm{GABA} / \mathrm{glycine}$ receptors in addition to postsynaptic scaffolding molecules $(\mathbf{C})$. Shown here are the interactions only with $\beta$-Neurexins, although Neuroligin variants can also bind $\alpha$-Neurexins to mediate effects at the synapse (see 1.1.4). 


\subsubsection{Neuroligins in synapse formation and function}

In vitro, the Neurexin-Neuroligin link can function as a bidirectional trigger to promote synapse initiation. Neuroligins present on the surface of fibroblasts trigger the formation of functional vesicle release sites in contacting axons (Scheiffele et al., 2000), and the synaptogenic activity of Neuroligins seems to rely on their association with Neurexins, as Neuroligin mutants that are deficient in $\beta$-Neurexin binding also fail to induce the formation of presynaptic terminals (Dean et al., 2003; Chubykin et al., 2005). Furthermore, Neurexins presented alone to dendrites can induce postsynaptic differentiation (Graf et al., 2004). Thus, in culture both proteins seem adequate for triggering hemisynapse formation. In accordance with a "synaptogenic" role for Neuroligins, overexpression of Neuroligins in neurons has been found in several studies to enhance the number of synapses (reviewed in Dean and Dresbach, 2006). Conversely, knockdown of individual Neuroligins by RNA interference in neuronal cultures leads to equivalent reductions in synapse number (Chih et al., 2005).

The in vivo study of Neuroligin function at the synapse by analysis of mutant mice deficient in one or more of the Neuroligin isoforms has only recently been addressed. The first such study analyzed mice lacking Neuroligins 1-3 (Varoqueaux et al., 2006). These mice die 24 hrs after birth due to respiratory failure. Upon functional analysis of the brainstem respiratory network (the pre-Botzinger complex and hypoglossal nucleus), a dramatic impairment of GABA/glycinergic transmission together with a moderate reduction of glutamatergic activity was observed. Interestingly, no alterations in synapse density or ultrastructure were observed at either excitatory or inhibitory synapses of the brain. There was, however, an impairment in the clustering of postsynaptic $\mathrm{GABA}_{\mathrm{A}}$ receptors $\left(\mathrm{GABA}_{\mathrm{A}} \alpha 1\right.$ subtype), with only little changes in the clustering of the inhibitory scaffold protein Gephyrin. These findings thus provided the first evidence that, in vivo, Neuroligins are crucial for synapse maturation (by recruitment of key synaptic components) and function, but not for the initial formation of synaptic contacts. Further, in keeping with the postsynaptic distribution of Neuroligin 1 (Song et al., 1999) and 2 (Varoqueaux et al., 2004), recordings from hippocampal slices of Neuroligin 1-deficient mice displayed a selective reduction in the NMDAR/AMPA ratio (Chubykin et al., 2007). Instead, Neuroligin 2 deletion specifically impaired evoked inhibitory responses in the barrel cortex (Chubykin et al., 2007). Developmentally, Neuroligin 2 clusters can be detected at very early stages of inhibitory postsynapse differentiation (Varoqueaux et al., 2004; Patrizi et al., 2008), indicating a central role of Neuroligin 2 in organizing inhibitory synapse assembly and function. 
Recent studies have focused on the role of the other two Neuroligin isoforms: Neuroligin 3 and 4. The synaptic morphology and function of the somatosensory cortex in Neuroligin 3deficient mice has been recently investigated (Tabuchi et al., 2007). The strength of evoked responses (both excitatory and inhibitory) analyzed for the Neuroligin 3-deficient mice revealed no alterations in inhibitory responses, but for a subtle (not significant) reduction in excitatory responses (Tabuchi et al., 2007). Further, morphological analysis revealed an intact synapse density (both excitatory and inhibitory) for the Neuroligin 3-deficient animals, as analyzed in brain sections from hippocampus and barrel cortex. These results warrant a thorough investigation into Neuroligin 3 distribution in the CNS, together with a systematic characterization of the Neuroligin 3 null mutant mice, to ascertain the synaptic specificities of this isoform.

New in vitro studies have focused on the effects of Neuroligin 4 overexpression in hippocampal cultures (Zhang et al., 2009). Although overexpression of Neuroligin 4, led to an increase in synapse number, it selectively suppressed the frequency of spontaneous (miniature) excitatory postsynaptic currents (mEPSCs), without having any effect on the amplitude of these currents or on miniature inhibitory postsynaptic currents (mIPSCs) (Zhang et al., 2009). Moreover, upon analysis of evoked EPSCs and IPSCs, it was observed that Neuroligin 4 overexpression selectively reduced the amplitude of the EPSCs. These results indicate a complex mechanism of action for Neuroligin 4 and it is hence, vital that the synaptic localization of Neuroligin 4 (both in vivo and in vitro) is carefully examined at cellular and subcellular levels simultaneously. In addition, it is important that the Neuroligin 4-deficient animals are thoroughly characterized, in order to dissect the precise role of Neuroligin 4 at CNS synapses. Furthermore, a detailed investigation into the exact role of Neuroligin 3 and 4 in the maintenance of synaptic organization and function is crucial, as these isoforms have been implicated in cognitive function.

\subsubsection{Neuroligins and cognitive function}

Mutations in the coding sequences of X-linked Neuroligin 3 and 4 have been identified in individuals with autistic disorder, Asperger syndrome and mental retardation (Jamain et al., 2003; Laumonnier et al., 2004; Yan et al., 2005). Autistic disorders are heritable neurodevelopmental syndromes characterized by impaired social interaction and communication, and restricted, stereotyped behavior (Association, 1994). Asperger syndrome is one of the autism spectrum disorders (ASD), characterized by higher cognitive abilities and more normal language function (Gillberg, 1998). Family and twin studies have conclusively 
described autism as the 'most genetic' neuropsychiatric disorder (reviewed in Persico and Bourgeron, 2006; Bourgeron, 2009). Interestingly, all the proteins implicated in ASDs so far, are involved in neurodevelopment and many have roles in synaptic function (reviewed in Persico and Bourgeron, 2006; Bourgeron, 2009), such as Neuroligins (Jamain et al., 2003; Laumonnier et al., 2004; Yan et al., 2005), Neurexins (Kim et al., 2008) and the scaffold protein SHANK3 (Durand et al., 2007). Moreover, some forms of autism are caused by an increased ratio of excitation/inhibition in sensory, mnemonic, social and emotional systems (reviewed in Rubenstein and Merzenich, 2003). Additionally, onset of ASDs occurs during the postnatal period of activity-dependent plasticity and remodeling of neuronal connections (Zoghbi, 2003), underscoring the crucial role of unbalanced excitatory-inhibitory networks in the pathogenesis observed in Autism (Levitt et al., 2004; Iacoboni et al., 2005).

The first mutations linking Neuroligins to autism were loss of function mutations in Neuroligin 3 and 4, found in siblings affected with ASDs (Jamain et al., 2003). A single mutation for Neuroligin 3 has been found in patients affected with ASDs (Jamain et al., 2003). This mutation converts a highly conserved arginine residue within the extracellular domain of the protein to cysteine (R451C), and the corresponding mutation in the mouse homologue of Neuroligin 3 (R471C), has been shown to cause misfolding and retention of the protein at the endoplasmic reticulum (Chih et al., 2004; Comoletti et al., 2004; Chubykin et al., 2005).

In contrast, several mutations in Neuroligin 4 have been identified in patients with ASDs (Jamain et al., 2003; Laumonnier et al., 2004; Yan et al., 2005; Zhang et al., 2009). The first mutation discovered was a frameshift mutation (1186insT), which resulted in premature termination of the protein (Jamain et al., 2003). Additional studies have discovered missense mutations in the acetylcholinesterase and cytoplasmic domain of Neuroligin 4 (Yan et al., 2005). Further, a two base pair deletion of the Neuroligin 4 gene has been found in individuals affected with autism, which caused the synthesis of a protein that lacked its C-terminal transmembrane domain (Laumonnier et al., 2004). Interestingly, this deletion was also present in nonautistic mentally retarded males indicating that mutations in Neuroligins could also be implicated in other cognitive impairments.

Even though many mutations have linked Neuroligin 4 to ASDs, mutated Neuroligin genes occur in $<1 \%$ of ASD diagnoses (Jamain et al., 2003; Laumonnier et al., 2004; Vincent et al., 2004; Gauthier et al., 2005; Yan et al., 2005). Yet they have provided crucial information on the synaptic abnormalities possibly present in ASD and it is thus vital to understand the roles of Neuroligin 3 and 4 in relation to synapse function and network activity. In this direction, a Neuroligin 3 Arg451Cys (R451C) knock-in mouse has recently been characterized (Tabuchi 
et al., 2007). This mutation depressed Neuroligin 3 abundance by $\sim 90 \%$, and led to major alterations in inhibitory synaptic transmission at the somatosensory cortex (Tabuchi et al., 2007). However, this mutation was considered to be a gain-of-function mutation as the Neuroligin 3-deficient mice were found not to display any of the features of the R451C mutation (discussed above in 1.1.5). Recent studies though, have also introduced the Neuroligin 3-deficient mouse as a model for the study of autism, as these mice were found to display reduced ultrasound vocalization and a lack of social novelty preference, reminiscent of the key symptoms of ASD (Radyushkin et al., 2009). Additionally, the mutant mice lacking Neuroligin 4 have similarly been shown to exhibit deficits in social interaction and communication characteristic of the symptoms observed for ASDs in humans (Jamain et al., 2008).

All these studies have brought to the field new transgenic tools, which can be used to understand the molecular mechanisms underlying cognitive disorders like autism. More importantly, the association of Neuroligins with cognitive disabilities underscores the necessity to evaluate the role of Neuroligins not only for the activity of a single synapse but also for the development and maintenance of neuronal networks. To address these questions, which were the primary focus of this study, the retina was chosen as the ideal model system. 


\subsection{The retina as a model system to explore synaptic function}

Visual processing begins at the retina, a multi-layered tissue that lies in front of the pigment epithelium lining the back of the eye. Devoted to the transduction, encoding, and transfer of visual information to higher brain areas, the retina is an extremely structured circuit (Wassle and Boycott, 1991; Boycott and Wassle, 1999; Masland, 2001b, a; Wassle, 2004), well suited for a detailed anatomical and electrophysiological investigation. It is therefore an optimal system to detect subtle impairments in synapse composition and function, network wiring and performance, and thus provides a suitable framework to explore the role of Neuroligins.

The retinal network is arranged such that a main excitatory glutamatergic pathway, sequentially involving photoreceptors, bipolars and ganglion cells, is modulated by distinct GABA and glycinergic inputs at two synaptic layers, the outer and inner plexiform layers. At the outer plexiform layer (OPL), horizontal cells modulate the activity of photoreceptor and bipolar dendrites, while diverse subclasses of amacrine cells modulate information processing at the inner plexiform layer (IPL). The myelinated axons of the ganglion cells form the optic nerve and carry the information to higher areas of visual processing (Figure 1.2).

At the inner synaptic layer of the retina, in contrast to other areas of the CNS, GABA and glycine receptors segregate into distinct synapse populations on amacrine, ganglion, and bipolar cell processes (Wassle et al., 1998; Haverkamp and Wassle, 2000). Moreover, the inhibitory scaffold protein Gephyrin is preferentially localized at glycinergic postsynapses in the retina and associates with only a subset of $\mathrm{GABA}_{\mathrm{A}}$ receptor clusters (Sassoe-Pognetto et al., 1995; Sassoe-Pognetto and Wassle, 1997). This unique compartmentalization of Gephyrin, GABA and glycine receptors in the retina hence provides an interesting framework for the characterization of Neuroligin isoforms.

\subsubsection{Information processing in the retina}

\subsubsection{Signal transmission}

Visual stimuli are converted to electrical signals by two types of photoreceptors: Rods, which mediate dim-light (or scotopic) vision and cones, which mediate color and bright light (or photopic) vision. In the mouse retina cones comprise only $\sim 3 \%$ of the total number of photoreceptors (Carter-Dawson and LaVail, 1979; Jeon et al., 1998), and are distributed in two populations of middle-wave (M) and short-wave (S) sensitive cones concentrated on the upper dorsal vs. lower ventral half of the retina, respectively (Szel et al., 1992). 
In the dark, the photoreceptors are in a depolarized state and constantly release glutamate. Upon light activation, visual pigments (Opsins) expressed at the photoreceptor outer segments induce a cascade of events ultimately leading to photoreceptor hyperpolarization and to the arrest of neurotransmitter release (for review see Stryer, 1991; Hargrave and McDowell, 1992).

The photoreceptor terminals are contacted by bipolar cell dendrites at the OPL. Bipolar cells have been categorized in several morphological subclasses; yet they are functionally classified as depolarizing "ON" rod bipolar cell (Berntson and Taylor, 2000; Euler and Masland, 2000), depolarizing "ON"- cone or hyperpolarizing "OFF"- cone bipolar cells. The differential response of ON vs. OFF bipolar cells lies in the distinct cohort of glutamate receptors that are accumulated at their dendritic tips, with the OFF-bipolar cells expressing ionotropic (iGLUR) glutamate receptors, while the ON-bipolar cells express the metabotropic mGLUR6 receptor (Nomura et al., 1994; Vardi et al., 2000). Interestingly, the axons of the two classes of bipolar cells also terminate at two distinct sublamina of the IPL, consequently termed ON and OFF sublamina, stratifying in the inner vs. outer part of the IPL respectively (Euler et al., 1996).

Bipolar cell axon terminals connect to ganglion cell dendrites at the IPL. About 10 different morphological types of ganglion cells have been shown to exist in the mammalian retina (Masland, 2001b), adding further diversity to the retinal architecture. Retinal ganglion cells can be classified functionally according to their light responses as ON-ganglion cells (those that spike at the onset of light), OFF-ganglion cells (which spike at the offset of light) and ON-OFF ganglion cells (which respond both to light onset and offset). In addition, these subsets of ganglion cells connect to bipolar cell terminals at the specified stratification (ON/OFF sublamina) of the IPL.

It is important to note that the only retinal neurons capable of generating action potentials are ganglion cells and some amacrine cells, the rest of the retinal neurons respond with graded changes in their membrane potential (non-spiking).

\subsubsection{Signal modulation}

At the OPL, the processes of horizontal cells form invaginating contacts onto photoreceptor terminals to modulate their activity. The rodent retina contains one type of horizontal cell (Peichl and Gonzalez-Soriano, 1994), an axon-bearing (B-type morphology) horizontal cell (Suzuki and Pinto, 1986) that connects to both rods and cones.

At the IPL, modulation of information is carried out by amacrine cells, of which as many as 50 different morphological subtypes have been reported (MacNeil and Masland, 1998). They 
can be broadly divided into two equally represented populations of wide-field GABAergic cells, and small field glycinergic cells (Pourcho and Goebel, 1985; Menger et al., 1998; reviewed in Wassle and Boycott, 1991).

A characteristic of GABAergic amacrine cells is the presence of other modulatory substances together with GABA at their processes (Vaney, 1990). Among them, cholinergic amacrine cells release acetylcholine in addition to GABA and are important for the direction selectivity circuit of the retina (reviewed in Wassle, 2004); dopaminergic amacrine cells, which release GABA and dopamine and have been implicated in the light adaptation process (reviewed in Witkovsky, 2004).

Glycinergic amacrine cells include at least ten different morphologic subtypes, of which the AII amacrine cells are the best studied. They are crucial intermediates in the rod pathway (Figure 1.2). They receive input from rod bipolar cells, and provide output onto ON-cone bipolar cells through gap junctions (at the ON sublamina) and onto OFF-cone bipolar cells through chemical synapses (at the OFF sublamina). The cone bipolar cells, in turn, transmit this information to the "output" stations (ON and OFF- ganglion cells respectively). In addition, a small proportion of AII cells (about 4\% in rabbit retina) make conventional synapses directly onto the dendrites of OFF-retinal ganglion cells (Strettoi et al., 1992; Murphy and Rieke, 2008). Thus, the AII amacrine cells couple the rod pathway to that of the cone system, as rod bipolar cells cannot directly transmit to ganglion cells (Famiglietti and Kolb, 1975; Raviola and Dacheux, 1987; Strettoi et al., 1990; reviewed in Kolb, 2006). This allows the rod pathway to take advantage of the complex operations of the cone bipolar circuitry (reviewed in Wassle, 2004). Yet, recent studies have provided evidence for alternative pathways for rod signal transmission. One such route is a direct gap-junction mediated contact between photoreceptors (DeVries and Baylor, 1995) and another pathway involves direct contact between the OFF-cone bipolar cell dendrites and the rod terminals at the OPL (Soucy et al., 1998; Hack et al., 1999; Tsukamoto et al., 2001). 
Pigment epithelium

Outer and Inner segments of photoreceptors

Outer Nuclear Layer

Outer Plexiform Layer

Inner Nuclear Layer

Inner Plexiform Layer

Ganglion cell Layer

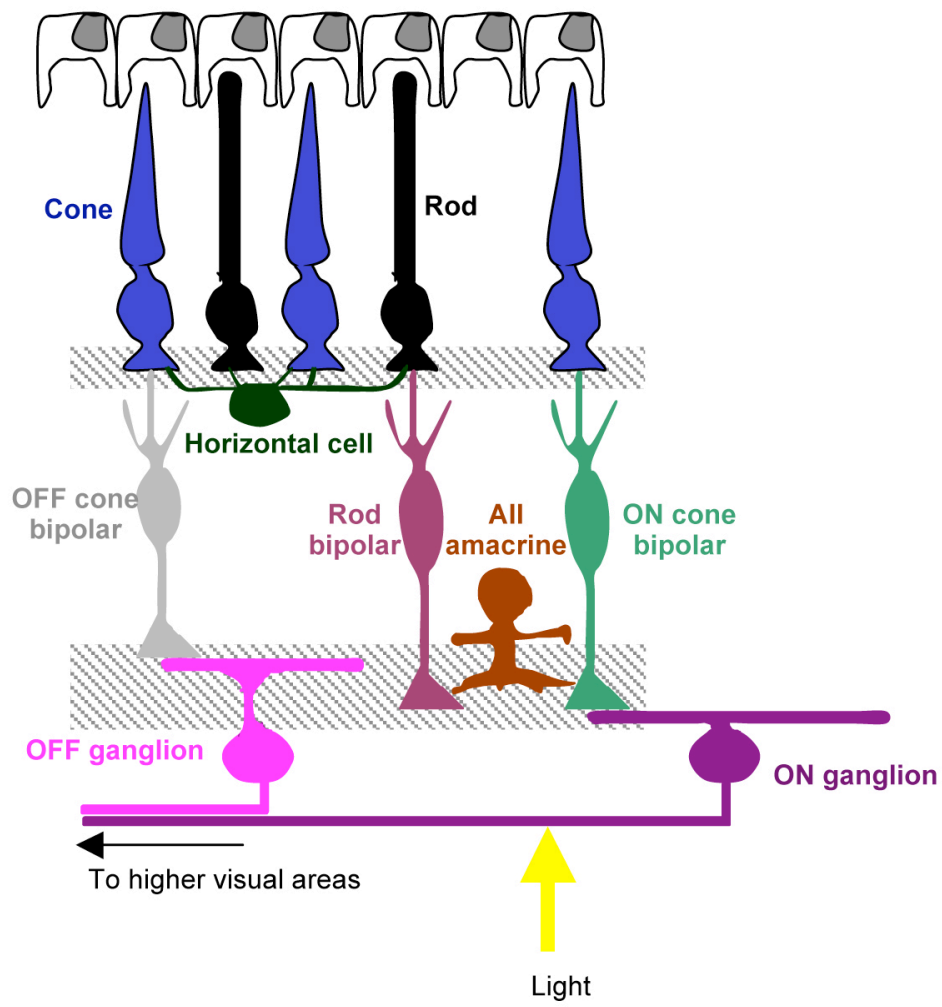

Figure 1.2: Information processing in the retina

Signal transduction occurs at the photoreceptors (rods and cones). Photoreceptor terminals contact bipolar cell dendrites and lateral horizontal cell processes at the OPL. The axon terminals of the bipolar cells then transmit the information at the IPL to the dendrites of the ganglion cells, which carry the message to higher visual centers. Rod bipolar cells, however, do not connect directly to the ganglion cells, but instead connect to glycinergic (AII) amacrine cells, which in turn, connect to (ON and OFF) cone bipolar cells. The bipolar cells thereafter transmit the information to ganglion cell dendrites. 


\subsubsection{Synaptic architecture of the retina}

Retinal neurons need to sustain high rates of transmission over long periods of time, for efficient sensory processing. This is enabled by the presence of specialized "ribbon" synapses in the retinal circuit, which have been shown to exocytose several hundreds of vesicles per second (far greater than the release at conventional synapses) (Heidelberger et al., 1994; von Gersdorff et al., 1996; Heidelberger et al., 2005). Ribbon synapses occur along the main transmission pathway, at the synapse between the photoreceptor and bipolar cell dendrites, and at contacts between bipolar cell terminals and ganglion cell dendrites. Ribbon synapses are also present in other sensory systems like the auditory and vestibular system, in fact, ribbons are employed wherever exocytosis is generated by graded depolarization and where signaling requires a high rate of sustained release (reviewed in Sterling and Matthews, 2005). Ultrastructual analyses have revealed that the ribbon is an electron-dense structure which holds synaptic vesicles close to the presynaptic membrane (reviewed in tom Dieck and Brandstatter, 2006). The molecular assembly of the presynaptic ribbon is largely unresolved and RIBEYE is the only known structural component of the ribbon (Schmitz et al., 2000). The cytomatrix protein Bassoon has been shown to link the ribbon to the active zone complex (Dick et al., 2003). In addition, ribbon synapses contain a different complement of presynaptic proteins (reviewed in Sterling and Matthews, 2005; tom Dieck and Brandstatter, 2006) and a specific non-inactivating calcium channel (L-type), which could promote tonic release of glutamate at the much lower calcium concentrations observed at the ribbon presynapse (reviewed in Morgans, 2000).

At the OPL of the mouse retina, several rod terminals (called rod spherules) are present together with a few large cone terminals (cone pedicle) (Figure 1.3A). The rod terminal consists of a single presynaptic ribbon which is apposed to invaginating contacts from horizontal cells and contact from a single invaginating (ON, mGLUR6 expressing) rod bipolar cell dendrite (Dowling and Boycott, 1966). This arrangement comprises the synaptic "triad" of the OPL. (Figure 1.3B-C). A cone pedicle on the other hand can contain several ribbons, and is contacted by two kinds of bipolar cell dendrites. Apart from the "triad", OFF cone bipolar cell dendrites (expressing iGLURs) form contacts at the base of each cone pedicle (reviewed in Wassle, 2004).

While it is known that horizontal cells receive information from photoreceptors and respond to light by hyperpolarization (by expression of iGLURs on their invaginating processes) (Brandstatter et al., 1997; Hack et al., 2001), how they mediate feedback onto photoreceptors is still debated. It has been shown that the horizontal cell processes are enriched with GABA 
containing vesicles (Vardi et al., 1994; Haverkamp et al., 2000; Cueva et al., 2002), which can provide feed-forward inhibition to GABA-receptor enriched bipolar cell dendritic processes (Haverkamp et al., 2000), but the direct feedback of horizontal cells onto photoreceptors has still not been confirmed in the mammalian retina. While, $\mathrm{GABA}_{\mathrm{A}}$ and $\mathrm{GABA}_{\mathrm{C}}$ mediated currents have been recorded from rat cone photoreceptors (Pattnaik et al., 2000), there is no direct ultrastructural evidence of GABA receptors being localized at the mammalian photoreceptor terminals, and no "classical" synapses have been found between horizontal cells and photoreceptor terminals in electron micrographs. Thus, alternative non-synaptic mechanisms of horizontal cell mediated feedback have been proposed (Kamermans et al., 2001; reviewed in Hirasawa and Kaneko, 2003; Wassle, 2004).

At the IPL of the mouse retina, the bipolar cell terminals provide excitatory input to amacrine cell processes and ganglion cell dendrites. In turn, amacrine cells modulate this information by providing feedback to the bipolar cell terminals and also by contacting ganglion cell dendrites and other amacrine cell processes (Figure 1.3D). There are two kind of synapses found in this retinal layer: the "ribbon" synapses present at the bipolar cell axon terminals (Figure 1.3E-F) and the "conventional" (inhibitory) synapses formed by the amacrine (GABA- and glycinergic) cells (Figure 1.3E,G).

Thus, the retina with its differential synaptic composition provides an interesting framework to investigate the distribution of Neuroligin isoforms. In this regard, the retina provides an opportunity to study if Neuroligins are needed for the formation/function of specialized ribbon synapses. Furthermore, in contrast to other regions of the CNS, distinct subsets of inhibitory GABA- and glycinergic synapses are localized at the IPL, which allows the characterization of Neuroligin isoforms at specified inhibitory synapses. 
A

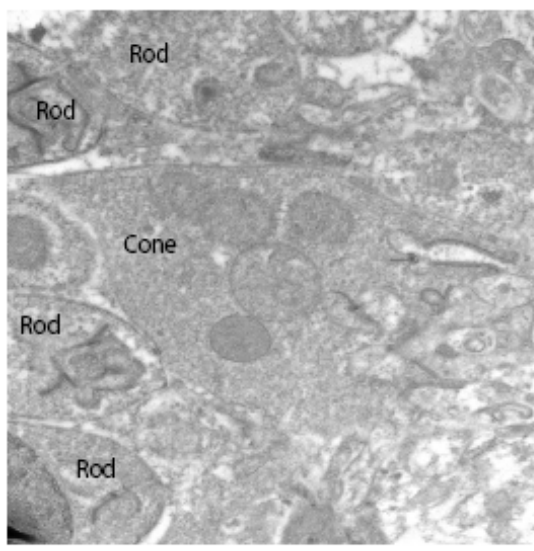

D

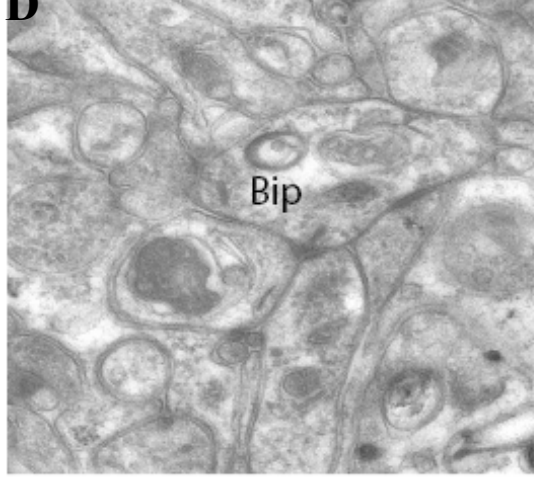

B
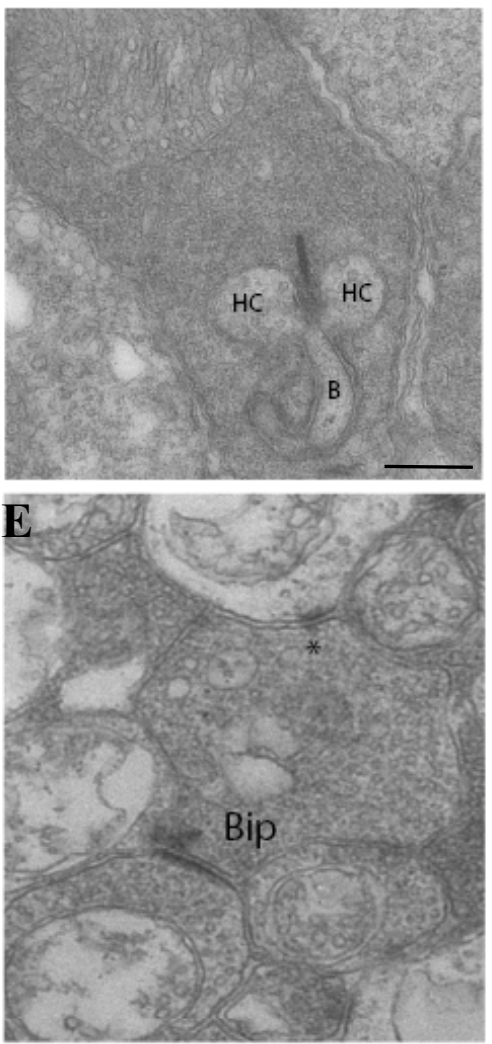

C

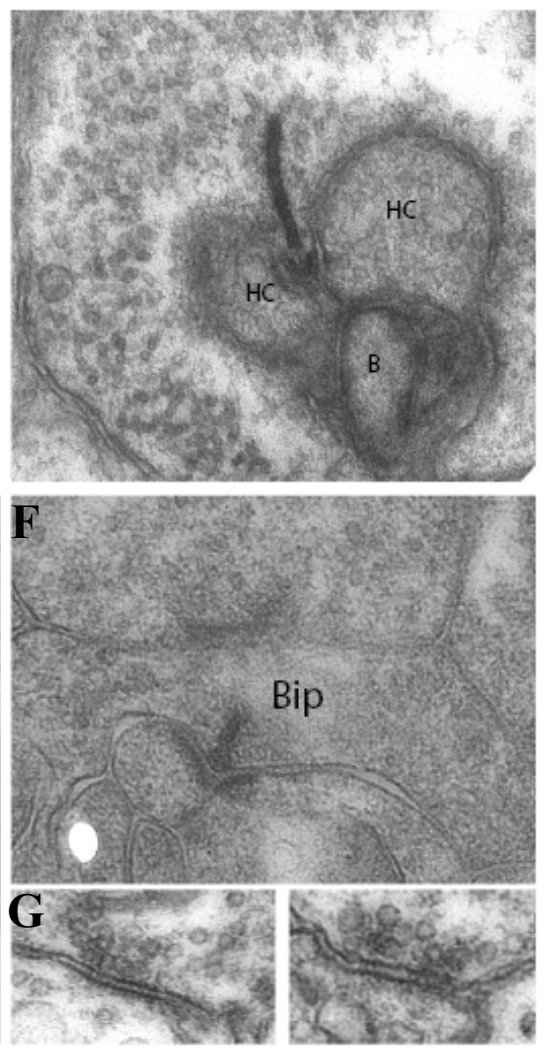

Figure 1.3: Synaptic architecture of the retina

At the OPL of the mouse retina, few cone pedicles (cone) are interspersed between several rod terminals (rod) (A). The ribbon synapse at the rod spherule connects with two invaginating processes from horizontal cells $(\mathrm{HC})$, together with a single invaginating process from a "depolarizing" bipolar (B) cell dendrite (B-C). At the IPL, the axon terminals of bipolar cells (Bip) display characteristic "ribbon" synapse (D-F) connections to amacrine cell processes or ganglion cell dendrites. Additionally, the bipolar cell terminals (Bip) also receive "conventional" inhibitory connections from amacrine cells (marked with *) (E, G). The inhibitory or symmetric synapses formed by the amacrine cells lack a prominent postsynaptic specialization $(\mathbf{G})$ and can mediate their effects by using glycine or GABA as neurotransmitter.

Scale bar $=450 \mathrm{~nm}$ 


\subsubsection{Inhibitory circuits in the retina}

GABA and glycine are the main inhibitory modulators at the IPL of the mouse retina (reviewed in Wassle and Boycott, 1991). Accordingly, distinct populations of GABA and glycine receptors are localized at the IPL. Glycine receptors (GlyR) are composed of ligandbinding $\alpha$ and $\beta$ subunits (Langosch et al., 1990; Betz, 1991). In adults, $\alpha$ - and $\beta$-subunits combine in a 2:3 ratio to form receptor pentamers (Grudzinska et al., 2005). At the postsynapse, GlyRs are believed to be clustered via their $\beta$-subunit by the 93-kDa scaffold protein Gephyrin (Kirsch et al., 1991; reviewed in Kneussel and Betz, 2000). Accordingly, there is a complete lack of GlyR clustering in the retinae from Gephyrin knock out mice (Fischer et al., 2000).

There are four genes encoding for the $\alpha$-subunit of GlyR, while only one gene codes for the $\beta$ subunit (Harvey et al., 2000). In the retina, all the four different isoforms of the $\alpha$-subunit $(\alpha 1$, $\alpha 2, \alpha 3, \alpha 4)$ are expressed, each with a characteristic distribution at the IPL (reviewed in Wassle et al., 2009). Colocalization studies have indicated that GlyR clusters in the retina are composed of only one type of $\alpha$-subunit, representing distinct inhibitory circuits at the IPL (Wassle et al., 2009). The GlyR $\alpha 1$ immunoreactivity is enriched at the outer IPL and represents synapses between the AII amacrine and the OFF cone bipolar cells (SassoePognetto et al., 1994). In addition, GlyR $\alpha 1$ puncta are also dispersed through the remainder of the IPL depicting glycinergic synapses onto ganglion cell processes and rod bipolar cell terminals (Ivanova et al., 2006; Majumdar et al., 2007). The GlyR $\alpha 2$ subunits are the most robustly expressed GlyR subunits in the retina and are spread throughout all laminae of the IPL (Haverkamp et al., 2004). GlyR $\alpha 2$ is expressed by amacrine and ganglion cell dendrites, but not by bipolar cell terminals (Ivanova et al., 2006). GlyR $\alpha 3$ is expressed in four distinct bands at the IPL and localized on the dendrites of the AII amacrine cells (Haverkamp et al., 2003; Weiss et al., 2008). Glycinergic synapses containing the GlyR $\alpha 4$ subunit have only been described in the inner synaptic layer of the retina (Heinze et al., 2007), where they are distributed in a single strata on the processes of the ON- (cholinergic) starburst amacrine cells. GABA is the other important modulator of visual processing and acts via a cohort of GABA receptors. There are three distinct types of $\mathrm{GABA}$ receptors $\left(\mathrm{GABA}_{\mathrm{A}}, \mathrm{GABA}_{\mathrm{B}}\right.$ and $\left.\mathrm{GABA}_{\mathrm{C}}\right)$ described in the $\mathrm{CNS}$. $\mathrm{GABA}_{\mathrm{A}}$ receptors are heteropentameric and formed by structurally related subunits (six $\alpha$, four $\beta$, three $\gamma$ and one $\delta$ ) (reviewed in Seeburg et al., 1990; Macdonald and Olsen, 1994; Sieghart, 1995). Using subunit specific antibodies distinct $\mathrm{GABA}_{\mathrm{A}}$ receptor subunits $(\alpha 1, \alpha 2, \alpha 3, \beta 1, \beta 2 / 3, \gamma 2$ and $\delta$ subunits $)$ have been localized at 
different strata of the IPL (reviewed in Wassle et al., 1998). The $\alpha 1, \beta 2 / 3$ and $\gamma 2$ subunits are the most abundantly expressed subunits in the retina (Wassle et al., 1998), with this combination of $\mathrm{GABA}_{\mathrm{A}}$ receptors being expressed extensively at bipolar cell terminals, amacrine cell processes and ganglion cell dendrites (Greferath et al., 1995). Distinct GABAergic synapses containing the $\alpha 1, \alpha 2$ and $\alpha 3$ subunits have been found on the dendrites of ganglion cells (Wassle et al., 1998). Cone bipolar cells and glycinergic amacrine cell processes have been shown to express the basic $\mathrm{GABA}_{\mathrm{A}}$ receptor type $(\alpha 1 \beta 2 / 3 \gamma 2)$, the rod bipolar cells to express $\alpha 1 / 3 \beta 2 / 3 \gamma 2$ subunit containing $\mathrm{GABA}_{\mathrm{A}}$ receptors, the cholinergic amacrine cells expressing unusual $\mathrm{GABA}_{\mathrm{A}}$ complexes $(\alpha 2 \beta 1 / 2 / 3 \delta)$ and the dopaminergic amacrine cells displaying the $\alpha 2 / 3$ and $\gamma 2$ subunit specific immunoreactivity (Greferath et al., 1993a; Greferath et al., 1993b; Greferath et al., 1995).

At the IPL, OFF-cone bipolar cells are dominated by glycinergic input, while GABAergic input dominates ON-cone and rod bipolar cells (Grunert, 2000). In contrast, there is a uniform distribution of GABA- and glycinergic input onto the dendrites of both ON and OFFganglion cells (Grunert, 2000) with no colocalization between $\mathrm{GABA}_{\mathrm{A}}$ and glycine receptors (Sassoe-Pognetto et al., 1995; Frazao et al., 2007).

In contrast to $\mathrm{GABA}_{\mathrm{A}}$ receptors in the brain, which rely on Gephyrin for postsynaptic clustering, a third of the $\mathrm{GABA}_{\mathrm{A}}$ receptors in the retina exhibit a gephyrin-independent mechanism of clustering (Fischer et al., 2000). Accordingly, Gephyrin has been found to colocalize with a subset of $\mathrm{GABA}_{\mathrm{A}}$ synapses in the retina. The most abundant colocalization has been observed with the $\alpha 2$ subunit followed by the $\alpha 3$ and $\alpha 1 \mathrm{GABA}_{\mathrm{A}}$ clusters (SassoePognetto et al., 1995). Accordingly, ultrastructural studies have found Gephyrin to be localized at amacrine and ganglion cell processes that express the $\mathrm{GABA}_{\mathrm{A}} \alpha 2$ subunit containing receptors, and not to be enriched at bipolar cell axons which predominantly express the $\mathrm{GABA}_{\mathrm{A}} \alpha 1$ subunit (Pourcho and Owczarzak, 1991; Grunert and Wassle, 1993; SassoePognetto et al., 1994).

$\mathrm{GABA}_{\mathrm{C}}$ receptors constitute the second class of ionotropic GABA receptors. They are homo/heteropentameric chloride channels expressed primarily in the vertebrate retina and are pharmacologically, molecularly and functionally distinct from the ionotropic $\mathrm{GABA}_{\mathrm{A}}$ receptors (reviewed in Lukasiewicz, 1996). Analysis of the response kinetics of $\mathrm{GABA}_{\mathrm{A}}$ and $\mathrm{GABA}_{\mathrm{C}}$ receptors revealed that $\mathrm{GABA}_{\mathrm{A}}$ components are brief with fast rise and decay time constants, whereas the $\mathrm{GABA}_{\mathrm{C}}$ components are more prolonged with slower time constants (reviewed in Lukasiewicz et al., 2004). Furthermore the two receptor classes have different 
affinities for GABA, with $\mathrm{GABA}_{\mathrm{C}}$ receptors being more sensitive than $\mathrm{GABA}_{\mathrm{A}}$ receptors in the retina (Feigenspan and Bormann, 1994). Interestingly, the protein MAP1B (microtubuleassociated protein $1 \mathrm{~B}$ ) has been shown to link $\mathrm{GABA}_{\mathrm{C}}$ receptors to cytoskeletal components (Hanley et al., 1999; Pattnaik et al., 2000) and also to modulate the functional properties of this receptor (Billups et al., 2000). The $\mathrm{GABA}_{\mathrm{C}}$ receptors are composed of the $\rho$ subunits, which have $\sim 35 \%$ homology to the $\mathrm{GABA}_{\mathrm{A}}$ receptor subunits (Cutting et al., 1991). GABA receptors are prominently expressed by rod bipolar cells (Enz et al., 1996; Koulen et al., 1997), independently from $\mathrm{GABA}_{\mathrm{A}}$ receptors (Koulen et al., 1998a), and largely determine their response properties (Eggers and Lukasiewicz, 2006).

Finally, inhibition can also be conveyed by G-protein gated $\mathrm{GABA}_{\mathrm{B}}$ receptors. $\mathrm{GABA}_{\mathrm{B}}$ receptors have a more prolonged response than $\mathrm{GABA}_{\mathrm{A}}$ receptors, and even though they both have the same affinity for GABA, weaker stimulus intensities are needed for activation of $\mathrm{GABA}_{\mathrm{A}}$ receptors as compared to $\mathrm{GABA}_{B}$ receptors, implying that $\mathrm{GABA}_{B}$ receptors might mediate the extrasynaptic action of GABA (Mody et al., 1994; Misgeld et al., 1995). In the vertebrate retina, $\mathrm{GABA}_{\mathrm{B}}$ receptors have been found postsynaptically on amacrine and ganglion cell processes (Slaughter and Pan, 1992). In addition, in the rat retina, $\mathrm{GABA}_{\mathrm{B}}$ receptors have also been localized presynaptically on horizontal (at OPL) and amacrine (at IPL) cell processes (Koulen et al., 1998c). However, further experiments are required to assess the contribution of $\mathrm{GABA}_{\mathrm{B}}$ receptors in retinal information processing.

Taken together, diverse populations of inhibitory synapses are localized in the retina, the functioning of which has a direct impact on visual information processing. This structurefunction relationship provided the ideal platform to investigate the role of Neuroligins, primarily Neuroligin 2, in the development, maintenance and functioning of a circuit. 


\subsection{Aim of the study}

The primary aim of this study was to take advantage of the well-structured retinal circuitry to explore the distribution of Neuroligins 2, 3 and 4 at the synapse, and to study their role in the establishment, maintenance and function of a neural network.

As the retina contains very diverse yet topologically distinct synaptic subtypes, the first task was to study the localization of the different Neuroligin isoforms at both light and electron microscope levels. Particularly, in regard to the association of Neuroligins with inhibitory synapses, the retina is an ideal model circuit, as it is one of the few regions of the CNS where GABA- and glycinergic synapses are differentially distributed and organized (see 1.2.3).

Next, morphological and functional approaches were used to evaluate the consequences of Neuroligin loss at single synapses, using single-isoform deletion mutant mice. One important question was to evaluate whether the lack of Neuroligins may lead to alterations in the distribution and composition of identified synaptic subtypes, and in the architecture of the retinal network as a whole. Further, to investigate functional alterations subsequent to Neuroligin deletion, multi-electrode arrays and, whenever possible, single cell patch-clamp recordings were performed to study the activity of ganglion cells, which convey information to higher visual areas.

Taken together, this study is the first to investigate systematically the localization and role of three Neuroligin isoforms in a morphologically intact model-system. 


\section{Materials and Methods}

All experiments were performed on 8 to 12 week-old adult wild-type (WT) and single deletion-mutant (KO) mice for Neuroligin 2, 3 and 4 (Varoqueaux et al., 2006; Jamain et al., 2008), in compliance with the guidelines for the welfare of experimental animals issued by the Federal Government of Germany, the NIH, and the Max Planck Society. Functional analyses were carried out on male age-matched littermates.

\subsection{Antibodies}

For characterizing the distribution of Neuroligins in the retina, immunolabelings were carried out using specific polyclonal rabbit antibodies against Neuroligin 2 (1:8000; Varoqueaux et al., 2004), Neuroligin 3 (1:3000; Varoqueaux et al., 2006) and Neuroligin 4 (1:2000; Jamain et al., 2008).

To detect GABA and glycine receptors, polyclonal guinea-pig antibodies against $\mathrm{GABA}_{\mathrm{A}} \alpha 1$, $\alpha 2, \alpha 3, \mathrm{GABA}_{\mathrm{A}} \gamma 2$ subunits $(1: 10000 ; 1: 5000 ; 1: 4000$; and 1:2500, respectively; Fritschy and Mohler, 1995; kindly provided by J.M. Fritschy, Zürich, Switzerland), a polyclonal rabbit antibody against GABA $_{C}$ (1:200; Fletcher et al., 1998; kindly provided by H. Wässle, MaxPlanck-Institute for Brain Research, Frankfurt, Germany), monoclonal mouse antibodies against all GlyR subunits or specific for GlyR $\alpha 1$ (mAb4a, 1:200; mAb2b, 1:500; Synaptic Systems, respectively), polyclonal goat antibodies against GlyR $\alpha 2$ and GlyR $\alpha 3$ (1:400 for both, Santa Cruz) and a polyclonal rabbit antibody against GlyR $\alpha 4$ (1:500, Chemicon) were used.

Specific retinal cell populations, ribbon synapses, excitatory and inhibitory pre- or postsynapses were detected with the following antibodies: Monoclonal mouse anti-PKC $\alpha$ (1:1000, Biodesign), -God (1:1000, Chemicon), -Calbindin (1:2000, Swant), -GAD65 (1:1000, Chemicon), -GAD67 (1:1000, Chemicon), -tyrosine hydroxylase (TH) (1:2000, Chemicon), -Bassoon (1:1000, Stressgen), -PSD95 (1:1000, Abcam), and -Gephyrin (3B11, 1:1000, Synaptic Systems); polyclonal rabbit anti-glycine transporter1 (GlyT1) (1:1000, kindly provided by N.C. Danbolt, University of Oslo, Oslo, Norway), -Neurokinin 3 receptor (NK3r) (a gift from E. Grady) (Grady et al., 1996), -vesicular acetylcholine transporter (VAChT) (1:2000, Synaptic systems), -Piccolo (1:1000, Synaptic Systems), -Synapsin (1:2000, Synaptic Systems), guinea pig anti-VGLUT1 (1:2000, Chemicon) or VIAAT (1:1000, Synaptic Systems). 
In addition for labeling the glycinergic amacrine cell population in Neuroligin 4 samples, a polyclonal goat anti-glycine transporter1 (1:1000, Chemicon) was used.

\subsection{Immunofluorescence labeling in the retina}

The animals were deeply anesthetized with Isofluran (DeltaSelect) and decapitated. The eyes were quickly removed, the lens dissected out and the eyecups immersed in $2 \%$ paraformaldehyde in $0.1 \mathrm{M}$ phosphate buffer, $\mathrm{pH} 7.4$ (PB) for 20 mins. Alternatively, the fixation time was reduced to 5 mins for GABA and glycine receptor labeling, or increased to 3 hrs for GlyT1 labeling with the commercial goat antibody. After fixation, the eyecups were rinsed in $\mathrm{PB}$, the retinae isolated and cryoprotected overnight in $30 \%$ sucrose in $\mathrm{PB}$. Alternating pieces of WT and KO retinae were frozen on top of each other in tissue freezing medium (Leica) to make "retina sandwiches". Briefly, the stage of the cryostat (Leica) was precooled to $-20^{\circ} \mathrm{C}$, and a retina piece was flattened on a parafilm-covered slide along with a drop of tissue freezing medium. The flattened retina sample was then frozen on the precooled stage, which already contained a base of tissue freezing medium. These retina sandwiches were then sectioned vertically at $14 \mu \mathrm{m}$ at the cryostat (Greferath et al., 1995).

For immunohistochemistry, sections were preincubated (for $1 \mathrm{hr}$ at room temperature) in PB containing $0.2 \%$ gelatin and $0.1 \%$ Triton X-100 (PGT), incubated overnight in primary antibodies in PGT followed by a $1 \mathrm{hr}$ incubation in secondary antibodies conjugated to either Alexa-488 or Alexa-555 (1:2000, Molecular Probes) in PGT. Fluorescein-Peanut Agglutinin (PNA, Vector) was used at a concentration of $5 \mathrm{mg} / \mathrm{ml}$.

\subsection{Electron Microscopy}

For photoreceptor quantification, animals were deeply anesthetized by intramuscular injection of tribromoethanol (TBE; stock $=4 \mathrm{mg} / \mathrm{ml}$; Sigma) and were subsequently perfused transcardially with a brief saline $(0.9 \%)$ flush followed by $2 \%$ paraformaldehyde, $2.5 \%$ glutaraldehyde and $0.3 \%$ tannic acid in PB for 10 mins. The retinae were isolated, washed, osmicated for $1 \mathrm{hr}\left(1 \% \mathrm{O}_{\mathrm{s}} \mathrm{O}_{4}\right.$ in $\left.\mathrm{PB}\right)$, dehydrated through graded series of ethanol and propylene oxide, and embedded in durcupan (ACM, Fluka). Semithin (500 nm) sections were collected and stained with methylene blue.

Postembedding immunolabeling was carried out following the procedure described by Matsubara et al., (1996) with minor changes. Briefly, retinae were prepared by freeze substitution and low-temperature embedding in Lowicryl HM20. Ultrathin sections (70- 
$90 \mathrm{~nm}$ ) were incubated overnight with the polyclonal rabbit anti-Neuroligin 2 or antiNeuroligin 4 antibody (both purified antibodies at a concentration of 1:100) in Tris-buffered saline with Triton $\mathrm{X}-100$ (TBST; $0.3 \% \mathrm{NaCl}$ and $0.01 \%$ Triton $\mathrm{X}-100$ ) containing $0.1 \%$ acetylated bovine serum albumin BSAc (Aurion). Secondary goat anti-rabbit IgG coupled to $10 \mathrm{~nm}$ gold particles (Aurion; 1:20) was used at a dilution of 1:20 in TBST containing 1\% BSA and polyethylene glycol $(5 \mathrm{mg} / \mathrm{ml}, 2 \mathrm{hrs})$. The sections were then contrasted with uranyl acetate and lead citrate and observed in a LEO 912AB transmission electron microscope (Zeiss). Digital images were taken using a ProScan CCD camera and analyzed with the AnalySIS software (Olympus).

\subsection{Imaging and Quantification}

For characterization of Neuroligin 2, immunofluorescent sections were viewed with an inverted confocal laser scanning microscope (LSM 510, Zeiss) and all images were acquired as single optical slices with a $40 \mathrm{X}$ water-immersion objective (NA 1.2) for illustration purposes and a digital zoom of 4.5 for quantification studies. During the characterization of Neuroligin 3 and 4, immunofluorescence was assessed by acquiring confocal images on an inverted TCS-SP2 confocal laser-scanning microscope (Leica Microsystems), with an oilimmersion $63 \mathrm{X}$ (NA 1.4) objective and a digital zoom factor of 4 for quantifications.

During acquisition, gain and offset were kept constant for a given labeling to allow for intensity comparisons between images. Images were further processed with AnalySIS software (Olympus). They were smoothened with an open filter and objects (i.e., fluorescent clusters, corresponding to synaptic puncta) were isolated upon application of a separation filter. In a selected region of interest (ROI) covering the entire width of the IPL, the number, size, gray value (minimum, maximum, mean) and integral intensity of all objects above an intensity threshold designated as background (set to a gray value of 50), and maintained constant for all images, were measured.

For colocalization studies, ROI were drawn manually for each fluorescent puncta in a given channel, superimposed on the complementary channel, and the number of colocalized puncta was determined manually (Fletcher et al., 1998). To account for the number of "random" associations between two markers, the ROI were superimposed on the complementary channel, which was first flipped horizontally. The number of colocalized ROI (puncta) with the flipped channel, thus, related to the number of random colocalizations between the two markers and was subtracted to represent the "true" associations. 
For photoreceptor quantification, semithin sections were observed with an upright Olympus BX-61 light microscope equipped with a $20 \mathrm{X}$ objective and images acquired with a B/W digital camera (F-ViewII) and analyzed with AnalySIS software (Olympus). The number of photoreceptors was determined manually by touch-count of photoreceptor nuclei in retinae stretches of comparable widths. For immunogold quantification, the number of gold particles at the synapse and the distance between the center of the gold particles and the center of the synapse (defined as the middle point of the postsynaptic membrane facing a presynaptic specialization) was measured.

\subsection{Immunohistochemistry and analysis of brain sections}

3 pairs of Neuroligin $2 \mathrm{WT}$ and KO littermates were anesthetized with TBE and perfused transcardially with 4\% paraformaldehyde, $0.1 \%$ glutaraldehyde in $0.1 \mathrm{M}$ PB for 5 mins. Brains were post-fixed for 10 mins in 4\% PFA, rinsed twice in PB, and cryoprotected overnight in $30 \%$ sucrose in PB. $40 \mu \mathrm{m}$ thick frontal sections were collected and further processed for immunostaining for VIAAT (polyclonal rabbit antibody from Synaptic Systems, 1:500) and Gephyrin (clone mAb7a, Synaptic Systems, 1:400) over a $48 \mathrm{hr}$ incubation in 2\% goat serum and $0.1 \%$ Triton $\mathrm{X}-100$ in $\mathrm{PB}$.

Alternatively, to allow for combined detection of VIAAT and the $\mathrm{GABA}_{\mathrm{A}} \gamma 2$ receptor subunit, other animals were used (for each genotype, $n=3$ ) and processed as described in Papadopoulos et al., (2007). Briefly, mice were deeply anesthetized with Isofluran (DeltaSelect), decapitated, the brains frozen on dry ice, and $14 \mu \mathrm{m}$-thick sections were cut at the cryostat. Sections were fixed by immersion in $4 \%$ paraformaldehyde for 10 mins and pretreated in sodium citrate buffer (10 mM sodium citrate, $0.05 \%$ Tween $20, \mathrm{Ph} 8)$ before permeabilization in $4 \%$ goat serum and $0.5 \%$ Triton X-100 in PB. Thereafter, sections were incubated overnight with antibodies against VIAAT (Rabbit, Synaptic Systems, 1:1000) and GABA $\gamma 2$ (1:4000, kindly provided by J.M. Fritschy, Zürich, Switzerland; Fritschy and Mohler, 1995) in 10\% NGS in PB.

Single-plane confocal images of immunolabeled sections were taken on an inverted TCS-SP2 confocal laser-scanning microscope (Leica Microsystems) with constant settings with a $63 \mathrm{X}$ objective and a zoom factor of 2 (or 4 for Gephyrin and VIAAT quantification at the stratum pyramidale), in the CA1 region. Quantification of immunofluorescence was performed with the AnalySIS software (Olympus) to assess the total number of VIAAT, Gephyrin and $\mathrm{GABA}_{\mathrm{A}} \gamma 2$ clusters separately at the strata radiatum and pyramidale. To detect these clusters, 
a particle separator was applied on a binarized image (obtained upon standard thresholding) and the number of puncta ascertained.

\subsection{Multi-electrode array (MEA) recordings}

These recordings were carried out in collaboration with Dr. Bjoern Falkenburger, at the Dept of Neurodegeneration and Restorative Research, University of Göttingen.

Recordings were performed using 200/30 MEAs (60 electrodes, $30 \mu \mathrm{m}$ diameter, $200 \mu \mathrm{m}$ spacing, 8x8 grid) and the MEA60BC amplifier (Multi Channel Systems). The animals were dark adapted ( $>6 \mathrm{hr}$ ) before experiments. Retinae were quickly dissected under dim red light and kept in oxygenated artificial $\mathrm{CSF}(\mathrm{aCSF})$ at $37^{\circ} \mathrm{C}$, containing in $\mathrm{mM}$ : $\mathrm{NaCl} 125, \mathrm{KCl} 2.5$, $\mathrm{CaCl}_{2} 2, \mathrm{MgCl}_{2} 1, \mathrm{NaHCO}_{2} 26, \mathrm{Na}_{3} \mathrm{PO}_{4} 1.25$, glucose 25 , equilibrated with $95 \% \mathrm{O}_{2}$ and $5 \%$ $\mathrm{CO}_{2}$. For recordings, retinae were placed with the ganglion cell layer facing the MEA. Potentials were digitized at $10 \mathrm{kHz}$ and high-pass filtered at $100 \mathrm{~Hz}$. To limit file size, $3 \mathrm{msec}$ spike cutouts were saved every time a threshold of $-10 \mu \mathrm{V}$ was crossed. These spike cutouts were sorted offline into individual units using Offline Sorter 2 (Plexon Inc). Light stimuli were delivered by a green LED placed at the camera port of an inverted microscope (BX-51, Olympus) triggered by a STG1004 stimulus generator and MC-Stimulus 2.0 (Multi Channel Systems). An ON/OFF stimulus, consisting of a $1 \mathrm{sec}$ light pulse was delivered 200 times. Cells were classified manually as "ON", "OFF" or "ON-OFF" blinded for genotype. Perievent-histograms (10 msec bins) were calculated by Neuroexplorer 3 (NEX technologies) and expressed either in spikes per second $(\mathrm{Hz})$ or in percentage of all spikes. Subsequent analysis and illustrations were carried out using IGOR Pro 5.03 (Wavemetrics). Statistical analyses were carried out with JMP 5 (SAS Institute Inc.) and Graph Pad Prism 4. Graphs represent mean \pm SEM.

\subsection{Patch clamp recordings from ganglion cells}

Whole-cell patch-clamp recordings from 3 week old (P22-P28) WT and Neuroligin 4-deficient ganglion cells were obtained from retinal whole-mounts. All recordings were carried out at room temperature, under dim-light conditions; and the animals were not dark adapted before the experiments.

Mice were deeply anesthetized with Isofluran (DeltaSelect), decapitated and retinae were dissected free of the eyecup in a low $\mathrm{Ca}^{2+}$ artificial CSF (aCSF) containing the following (in $\mathrm{mM}): \mathrm{NaCl} 125, \mathrm{KCl} 2.5, \mathrm{MgCl}_{2} 1, \mathrm{CaCl}_{2}$ 0.5, glucose 25, $\mathrm{NaHCO}_{3} 25, \mathrm{NaH}_{2} \mathrm{PO}_{4} 1.25$, 
ascorbic acid 0.4, myo-inositol 3, Na-pyruvate 2, pH 7.3 when bubbled with carbogen $(95 \%$ $\mathrm{O}_{2}, 5 \% \mathrm{CO}_{2}$ ). Retinae were mounted onto the experiment chamber with the ganglion cell layer facing up and the cells were visualized by infrared-differential interference contrast microscopy through a $60 \mathrm{X}$ water-immersion objective (Olympus, NA 1.00) using an upright microscope (Axioskop FS, Zeiss) equipped with a CCD camera (Till photonics). Before recording from ganglion cells the inner limiting membrane was gently removed with a glass pipette (Majumdar et al., 2007).

The intracellular solution contained, in mM: Cs-Gluconate 55, $\mathrm{CsCl}_{2} 55, \mathrm{CaCl}_{2}$ 1, EGTA 10, Na-Hepes 10, Mg-ATP 4, Na-GTP 0.4, Alexa 488 (Molecular Probes) 0.1, pH = 7.3.

The recordings were carried out with an EPC-10 patch-clamp amplifier (HEKA Elektronik, Germany) and Pulse software. Patch pipettes were pulled on a PIP 5 vertical puller (HEKA, Elektronik, Germany) from borosilicate glass tubing (Hilgenberg, Germany). When filled with internal solution, they had a resistance of 4-6 M . Series resistance was $<20 \mathrm{M} \Omega$ and routinely compensated (42\%) during experiments. The signals were filtered using the eightpole Bessel filter, using a cut-off frequency $\mathrm{f}_{\mathrm{c}}=5 \mathrm{kHz}$. The sampling rate during recordings was $20 \mathrm{kHz}$.

In order to distinguish retinal ganglion cells from "displaced" amacrine cells also localized at the ganglion cell layer, only cells with a diameter larger than $15 \mu \mathrm{m}$ were recorded from (Rothe et al., 1999; Majumdar et al., 2007; Majumdar et al., 2009). Further only those cells generating voltage-activated sodium currents $\left(\mathrm{I}_{\mathrm{NA}}\right)>2 \mathrm{nA}$ were considered as ganglion cells (Rothe et al., 1999). Moreover, the recorded cells were visualized to ascertain whether they had an axon projecting away from the ganglion cell layer- another distinguishing feature of retinal ganglion cells.

While recording glycinergic mIPSCs, TTX $(1 \mu \mathrm{M})$ was applied to the bath to prevent the generation of action potentials (APs). Glycinergic mIPSCs were recorded at $\mathrm{V}_{\text {holding }}=-70 \mathrm{mV}$, in $\operatorname{aCSF}\left(0.5 \mathrm{mM} \mathrm{Ca}^{2+}, 1 \mathrm{mM} \mathrm{Mg}^{2+}\right)$ in the presence of NBQX $(1 \mu \mathrm{M})$ AP5 $(50 \mu \mathrm{M})$ and bicuculline $(50 \mu \mathrm{M})$. $\mathrm{I}_{\mathrm{NA}}$ was recorded from each cell before adding TTX to the external solution.

In the absence of TTX, ganglion cells displayed very robust GABAergic mIPSC activity, which frequently occurred in bursts. This activity typically continued for several minutes even after washing-in TTX. Because the high frequency of events hampered the analysis of individual mIPSCs, we generally bathed the retinae in TTX-supplemented external solution while recording GABAergic mIPSCs, as TTX has been shown to reduce significantly the 
frequency of GABAergic mIPSCs but not glycinergic mIPSCs (Protti et al., 1997) in ganglion cells recorded from rat retinal slices.

Nevertheless, to ensure that GABAergic mIPSCs were recorded exclusively from ganglion cells, once again only cells larger than $15 \mu \mathrm{m}$ in diameter were selected from the ganglion cell layer for recording and their identification as ganglion cells was confirmed by examining the cells for the presence of an axon projecting away from the ganglion cell layer. GABAergic mIPSCs were also recorded at a holding potential of $-70 \mathrm{mV}$ in aCSF containing strychnine (500 nM), NBQX and AP5.

Once in the whole-cell configuration, images of the cell were taken with a fluorescence microscope to determine the presence of an axon, which is characteristic of ganglion cells. This was further ratified after recording when the retina slice was fixed for 15 mins in $2 \%$ paraformaldehyde (PFA) in PB, rinsed, mounted on a slide and viewed on an inverted TCSSP2 confocal laser-scanning microscope (Leica Microsystems).

Data analysis was carried out in IGOR Pro 6.1 (Wavemetrics, Lake Oswego, OR). mIPSCs were detected using a sliding template algorithm (Clements and Bekkers, 1997). Peak amplitudes, decay time constants (a single exponential was used to fit the decay time constants for mIPSCs) (Majumdar et al., 2007) and (20-80\%) rise times were estimated from the average mIPSC trace estimated from individual cells. For comparison, the frequency distributions of decay time constants and their average values were also obtained from fitting exponentials to individual mIPSCs. Only events with peak amplitudes $\geq 15 \mathrm{pA}$ were included in this analysis, as smaller events did not allow reliable fits.

Data analysis of the mIPSCs was carried out in collaboration with Dr. Holger Taschenberger, at the Department of Membrane Biophysics, Max Planck Institute of Biophysical Chemistry.

\subsection{Statistics}

As all the data passed the normality test, statistical analyses between genotypes were carried out using the two-tailed paired t-test with Welch correction.

$*, \mathrm{p}<0.05, * *, \mathrm{p}<0.01, * * *, \mathrm{p}<0.001$ 


\section{Results}

\subsection{Distribution of Neuroligin isoforms in the mouse retina}

To characterize the distribution of Neuroligins 2-4 in the mouse retina, isoform-specific antibodies were used. The specificity of the antibodies was determined by labeling wild-type retina sections together with samples from the respective isoform-specific deletion mutants. As illustrated in Figure 3.1, Neuroligins 2, 3 and 4 are abundantly expressed in the mouse retina, with the labeling being concentrated at the IPL. The antibodies used are indeed specific as no signal can be detected from the respective knockout retina sections (Figure 3.1A-C). Interestingly, Neuroligin 2 positive puncta are also present at the OPL of the retina (Figure 3.1A), indicating that in addition to potentially participating in visual processing at the IPL, Neuroligin 2 might also affect processing between photoreceptors and bipolar cell dendrites. Further, Neuroligin 4 is rather sparsely distributed at the IPL, in comparison to Neuroligin 2 and 3, which are densely clustered throughout the IPL (Figure 3.1C). In addition, the labeling for Neuroligin 4 appears to be concentrated at the outer (OFF) sublamina of the IPL (Figure 3.1C) and is rather scarce through the rest of the IPL. 
A

B

NL2 WT

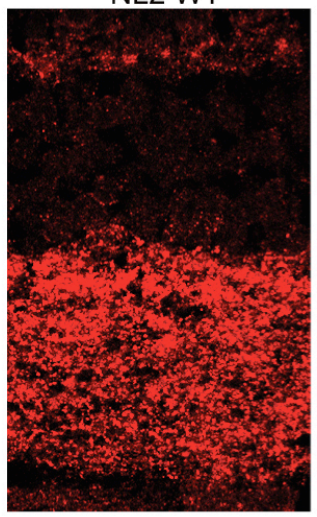

C

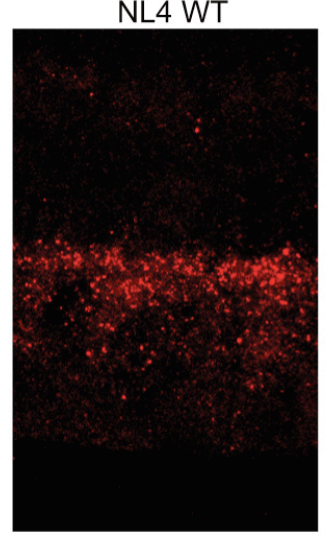

$\mathrm{NL} 2 \mathrm{KO}$

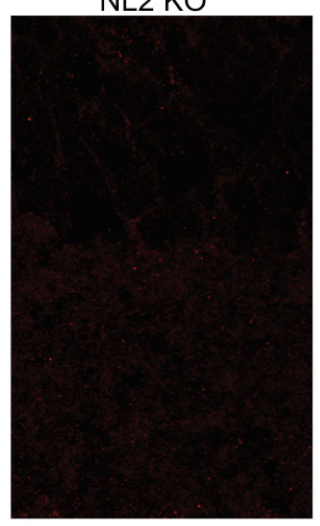

NL4 KO
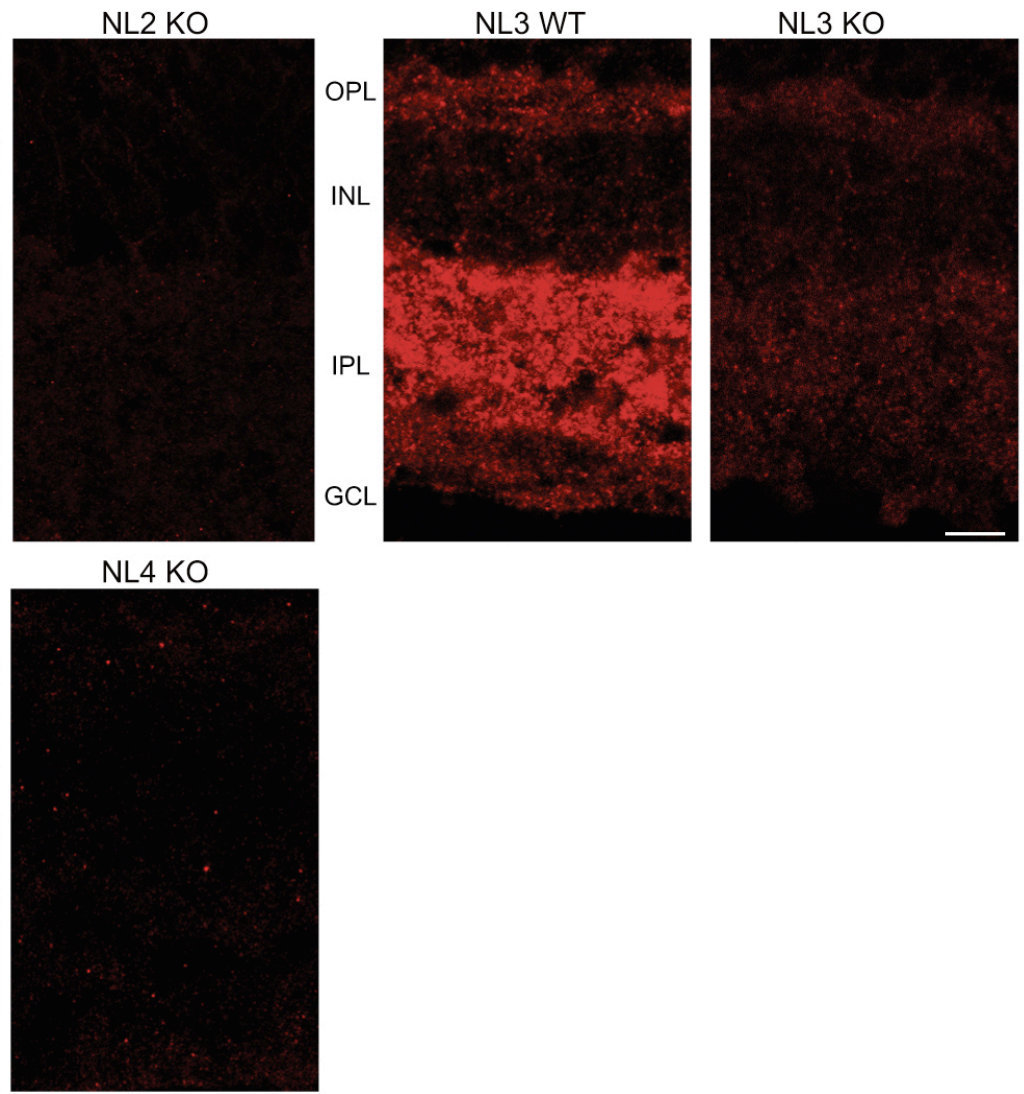

Figure 3.1: Expression of Neuroligin isoforms in the mouse retina

Isoform-specific antibodies were used to study the expression of Neuroligins in the retina. Neuroligin 2 (NL2) was localized at both the OPL and IPL (A). Neuroligin 3 (NL3) was also expressed robustly at the inner synaptic layer of the retina (B). However, Neuroligin 4 (NL4) was concentrated at the outer lamina of the IPL with a rather sparse distribution through the rest of the IPL (C). The specificity of the antibodies is illustrated by the absence of immunoreactivity in the corresponding isoform-specific deletion mutants (KO) as compared to wild-type (WT) retinae.

OPL, outer plexiform layer; INL, inner nuclear layer; IPL, inner plexiform layer; GCL, ganglion cell layer.

Scale bar $=5 \mu \mathrm{m}$. 


\subsection{Functional characterization of Neuroligin 2}

The first part of the study investigated the distribution and function of Neuroligin 2 in the mouse retina. Earlier studies have shown that Neuroligin 2 is localized at all inhibitory synapses in the brain (Varoqueaux et al., 2004).

For a detailed characterization of Neuroligin 2 distribution in the retina, the isoform-specific antibody (Figure 3.1) was used in combination with a wide array of different pre- and postsynaptic markers. Further, to study how retinal architecture and function is affected in the absence of Neuroligin 2, retinae from pairs of wild-type and Neuroligin 2-deficient animals were analyzed.

\subsubsection{Neuroligin 2 is preferentially localized to $\mathrm{GABA}_{\mathrm{A}}$ receptor containing synapses}

In wild-type retinae, Neuroligin 2 yielded an abundant punctate labeling throughout the OPL and IPL (Figure 3.1A). A series of colocalization experiments with landmark postsynaptic receptor and scaffold proteins was performed to assess whether Neuroligin 2 is specifically segregated to certain synaptic subtypes of the retina (Figure 3.2). Neuroligin 2 never colocalized with the ubiquitous excitatory postsynaptic scaffold protein PSD-95 (Figure 3.2B, E), demonstrating its general absence from glutamatergic postsynaptic sites. Thus, in the retina, as in the brain, Neuroligin 2 is devoid from excitatory postsynapses.

In the retina, inhibition is mediated by GABAergic horizontal cells at the OPL, and by GABAand glycinergic amacrine neurons at the IPL. Their processes contact postsynaptic specializations that bear segregated populations of $\mathrm{GABA}_{\mathrm{A}}$ and glycine receptors, respectively. $\mathrm{GABA}_{\mathrm{C}}$ receptors are also expressed, and cluster mainly at bipolar cell axon terminals (Wassle et al., 1998). Multiple $\mathrm{GABA}_{\mathrm{A}}$ receptor subtypes have been described; eight $\mathrm{GABA}_{\mathrm{A}}$ receptor subunits $(\alpha 1-3 ; \beta 1-3 ; \gamma 2$ and $\delta)$ are expressed in various combination and are localized at characteristic strata of the IPL (Greferath et al., 1995). The GABA $\gamma^{2} 2$ subunit is the core component of a majority of $\mathrm{GABA}_{\mathrm{A}}$ receptors in the retina as is the case in the brain (Fritschy and Mohler, 1995; Wassle et al., 1998). Virtually all Neuroligin 2 puncta were also positive for GABA $\mathrm{A}_{\mathrm{A}} 2$ at both the OPL and IPL (Figure 3.2A, E), indicating that, in the retina, Neuroligin 2 is associated extensively with GABAergic postsynaptic specializations.

Conversely, only half of Neuroligin 2-positive puncta at the IPL were also positive for the inhibitory scaffold protein Gephyrin (Figure 3.2C, E), indicating that Neuroligin 2 does not associate with all inhibitory postsynapses in the retina. In turn, 45\% of Gephyrin clusters colocalized with Neuroligin 2 (Figure 3.3D). In the retina, Gephyrin has been shown to 
mediate the clustering of all GlyRs (Fischer et al., 2000), and to associate with about twothirds of $\mathrm{GABA}_{\mathrm{A}}$ receptor clusters, depending on their composition (Sassoe-Pognetto et al., 1995). This observation prompted the examination of Neuroligin 2 colocalization with GlyRs. Indeed, Neuroligin 2 was present at only a minor fraction of glycinergic postsynapses that were detected with an antibody recognizing both $\alpha$ and $\beta$ GlyR subunits (mAb4a; Kirsch and Betz, 1993; only 19\% of Neuroligin 2-positive puncta were mAb4a-positive) (Figure 3.2D, E). In summary, the above results indicate that Neuroligin 2 is specifically localized at GABAergic postsynapses of the retina. However, not all GABAergic synapses were Neuroligin 2 positive, as only two-thirds of GABA $\gamma 2$ puncta associated with Neuroligin 2 (Figure 3.3D). This raised the possibility that Neuroligin 2 might associate preferentially with specific subsets of inhibitory GABAergic synapses, that can be identified by immunolabeling for $\mathrm{GABA}_{\mathrm{A}} \alpha 1, \alpha 2$ or $\alpha 3$ subunits (Koulen et al., 1996). Therefore, double labelings were carried out for Neuroligin 2 and each of these subunits (Figure 3.3A-C). Neuroligin 2 colocalized quite extensively with $\mathrm{GABA}_{\mathrm{A}} \alpha 1$ (58\% of Neuroligin 2 puncta, Figure 3.2E, Figure 3.3A), but also significantly with $\mathrm{GABA}_{\mathrm{A}} \alpha 2(43 \%$, Figure 3.2E, Figure 3.3B) and $\mathrm{GABA}_{\mathrm{A}} \alpha 3(45 \%$, Figure 3.2E, Figure 3.3C) receptor clusters. Overall, these findings show that Neuroligin 2 function is not limited to a specific subset of GABAergic synapses but instead distributed over the entire $\mathrm{GABA}_{\mathrm{A}}$ ergic circuit.

At the ultrastructural level, the presence of Neuroligin 2 at inhibitory, symmetric synapses of the IPL was confirmed by postembedding immunogold labeling (Figure 3.4A-C). The tangential distribution of Neuroligin 2-related gold particles within $100 \mathrm{~nm}$ from the center of the postsynaptic membrane (Figure 3.4A-C) resembles the distribution pattern of $\mathrm{GABA}_{\mathrm{A}}$ receptor subunits in other brain regions (Panzanelli et al., 2004). These results provided further evidence that Neuroligin 2 is localized specifically at the inhibitory postsynaptic compartment. Taken together, the characterization of Neuroligin 2 distribution in the retina revealed its specific association to $\mathrm{GABA}_{\mathrm{A}}$ receptor containing synapses, with only a minor fraction of Neuroligin 2 present at GlyR postsynapses. 


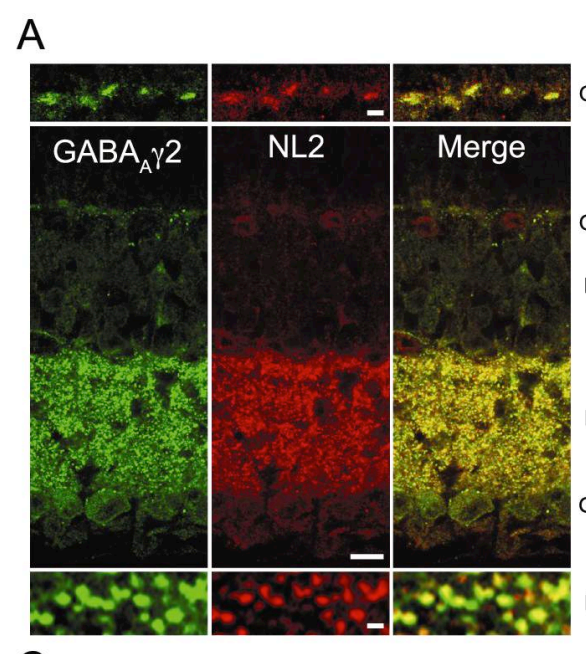

B

C
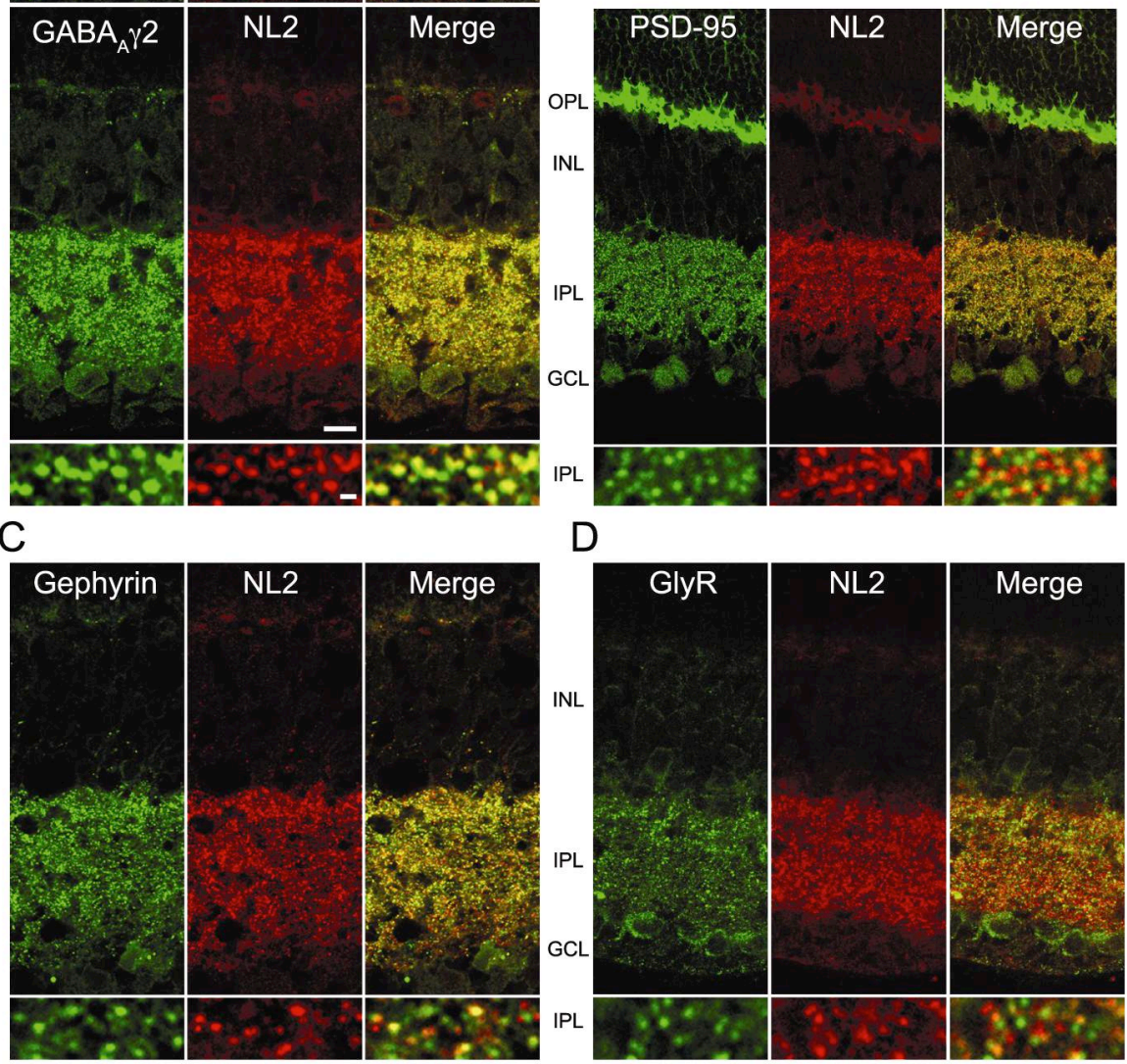

D

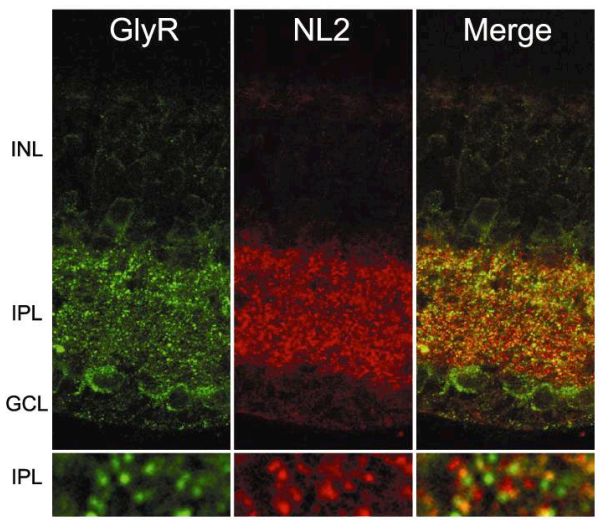

E

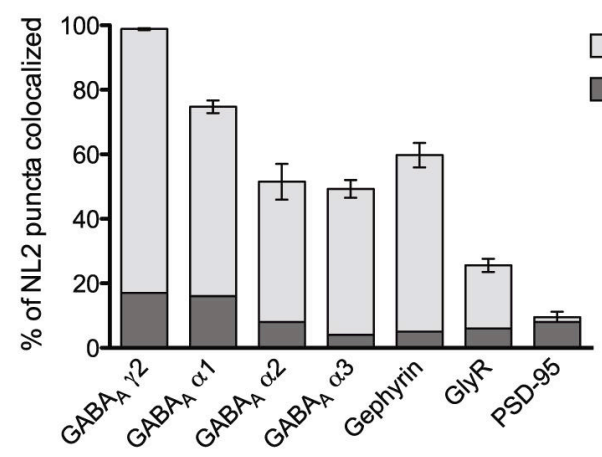

Specific overlap

Random overlap (flipped image)

Figure 3.2: Neuroligin 2 is localized preferentially at GABAergic postsynapses of the retina

Neuroligin 2 (NL2) puncta colocalized extensively with $\mathrm{GABA}_{\mathrm{A}} \gamma 2$ receptor subunits at both the plexiform layers of the retina (A). Conversely, Neuroligin 2 puncta did not associate with the excitatory postsynaptic marker PSD-95 (B). About half of Neuroligin 2 puncta associated with the inhibitory scaffold protein Gephyrin (C), indicating that Neuroligin 2 may not be present at all inhibitory postsynapses of the retina. Accordingly, a restricted colocalization was observed for Neuroligin 2 with GlyR subunits (D). Quantifications sustained these results (E), and a majority of Neuroligin 2 puncta colocalized with $\mathrm{GABA}_{\mathrm{A}}$ receptor subunits. OPL, outer plexiform layer; INL, inner nuclear layer; IPL, inner plexiform layer; GCL, ganglion cell layer. Scale bars = overview, $10 \mu \mathrm{m}$, detail, $1.5 \mu \mathrm{m}$ in OPL panel, $0.6 \mu \mathrm{m}$ in IPL panel.

Adapted from Hoon et al., 2009. 
A

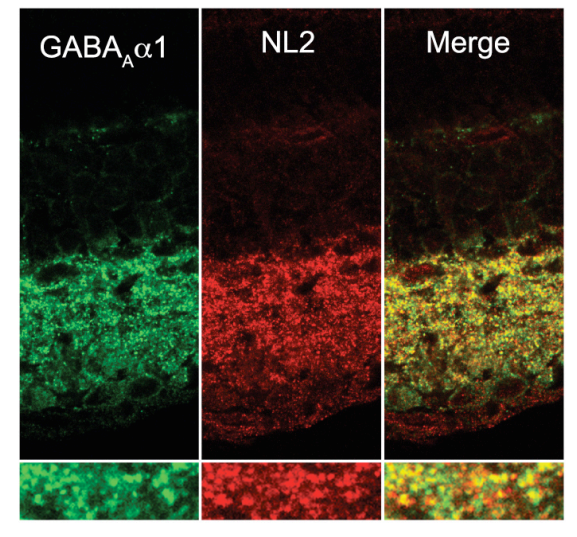

C

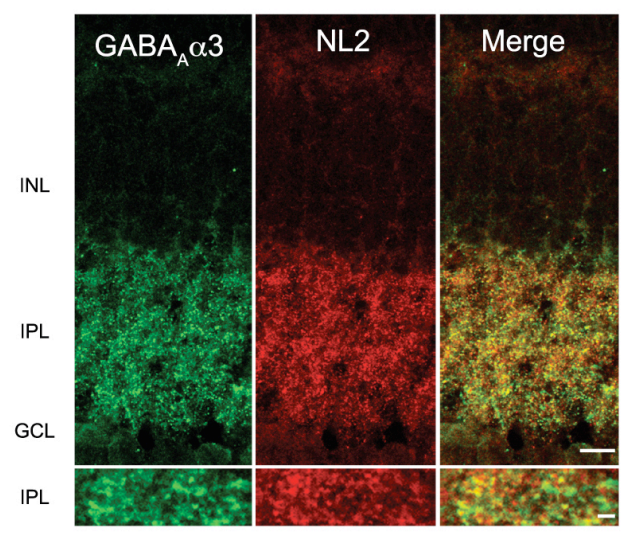

B

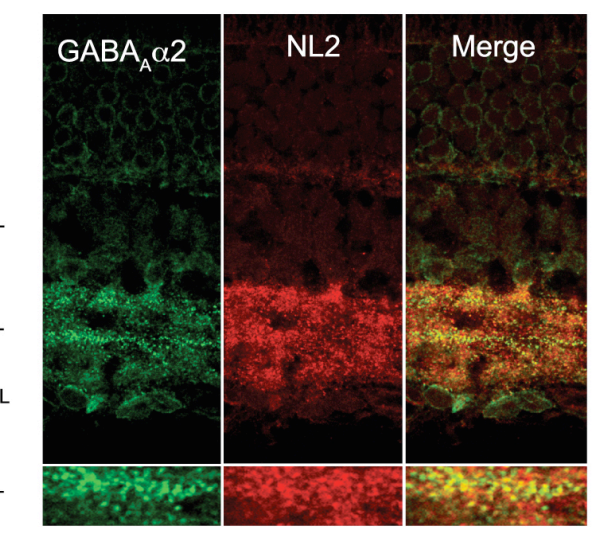

D

\begin{tabular}{|c|c|c|}
\cline { 2 - 3 } \multicolumn{1}{c|}{} & $\begin{array}{c}\text { \% coloc with NL2 } \\
\text { (specific superposition) }\end{array}$ & \pm SEM \\
\hline GABA $_{A} \gamma 2$ & 58 & 3.83 \\
\hline GABAA $_{2} \alpha 1$ & 65 & 4.10 \\
\hline GABA $_{A} \alpha 22$ & 66 & 3.17 \\
\hline GABA $_{A} \alpha 3$ & 70 & 3.23 \\
\hline Gephyrin & 45 & 2.58 \\
\hline GlyR & 16 & 0.92 \\
\hline PSD-95 & 3 & 1.65 \\
\hline
\end{tabular}

Figure 3.3: Association of Neuroligin 2 with different $\mathrm{GABA}_{\mathrm{A}}$ receptor subsets

Neuroligin 2 (NL2) associates with different subsets of GABAergic synapses in the retina as indicated by a significant overlap of Neuroligin 2 with the GABA $\alpha 1(\mathbf{A}), \alpha 2(\mathbf{B})$, or $\alpha 3$ (C) receptors subunits.

(D) Proportion of GABA $\mathrm{A} \gamma 2, \alpha 1, \alpha 2, \alpha 3$, Gephyrin, GlyR and PSD-95 immunoreactive clusters that colocalize with Neuroligin 2 at the IPL $(n=5)$.

INL, inner nuclear layer; IPL, inner plexiform layer; GCL, ganglion cell layer.

Scale bars $=$ overview, $10 \mu \mathrm{m}$, detail, $1 \mu \mathrm{m}$.

Adapted from Hoon et al., 2009. 


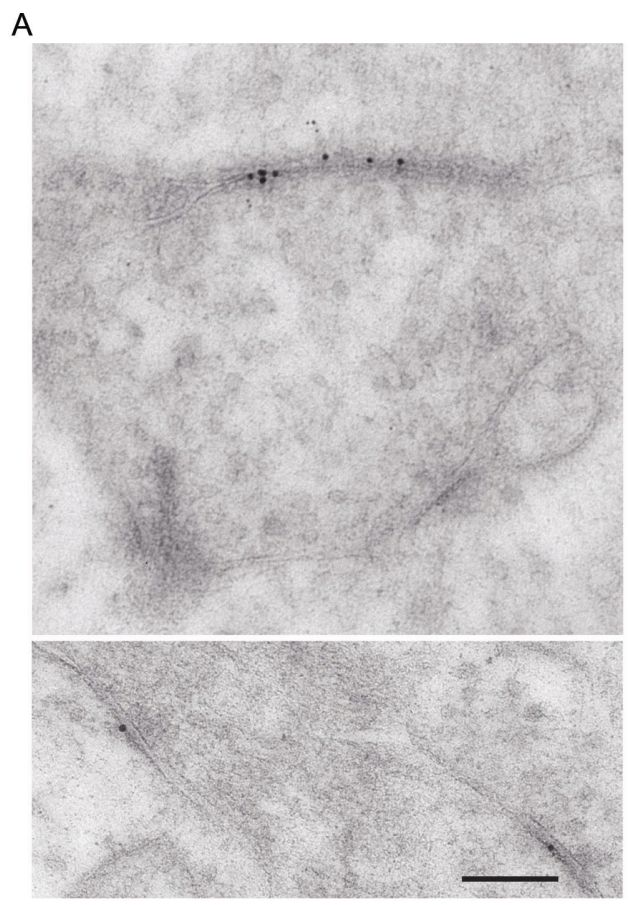

Figure 3.4: Ultrastructural localization of Neuroligin 2 at symmetric synapses of the IPL

Upon postembedding immunolabeling, gold particles corresponding to Neuroligin 2 were located specifically at inhibitory (symmetric) (A) but not at excitatory (asymmetric) synapses of the IPL (note for example the absence of labeling at a bipolar ribbon synapse, top micrograph).

Albeit in varying amount, they were repeatedly observed at symmetric synapses of the IPL (bottom micrograph, and quantified in B), where they spread tangentially from the center of the synapse $(\mathbf{C})$. Scale bar $=500 \mathrm{~nm}$.

Adapted from Hoon et al., 2009.

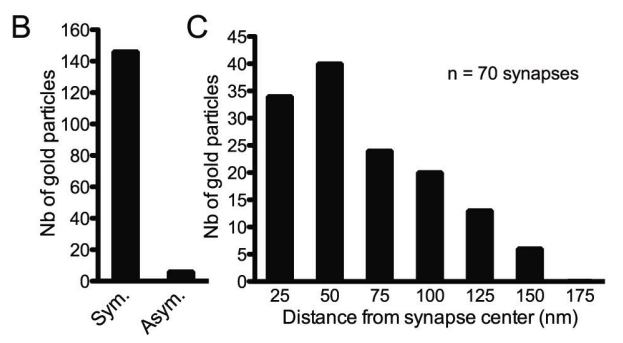

\subsubsection{Neuroligin 2 deficiency does not affect the core architecture of the retina}

The relay between amacrine and ganglion cells constitutes the first functional synaptic circuit in the retina, and is key to establishing proper retinal connectivity (Mumm et al., 2005). $\mathrm{GABA}_{\mathrm{A}}$ receptors are important components of this circuitry and modulate network excitability in the developing retina (Wang et al., 2007). The immature retina spontaneously generates "waves" of rhythmic bursting activity that sculpts the connectivity pattern of retinal ganglion cells, and GABAergic transmission plays an important role in modulating this bursting activity of ganglion cells (reviewed in Wong, 1999).

Previous studies in the brain have shown Neuroligin 2 to be present at the earliest stages of synapse development, and to be required for early synapse maturation (Graf et al., 2004; Varoqueaux et al., 2006; Chubykin et al., 2007). Thus, it was crucial to examine whether the deletion of Neuroligin 2 would affect the organization of the mature retinal circuitry. Hence, 
the abundance of major cell and synapse populations in Neuroligin 2-deficient (KO) and wildtype (WT) retinae were compared.

The entire width of the retina (Figures 3.5, 3.6) and the relative sizes of nuclear and synaptic (plexiform) layers were comparable in Neuroligin $2 \mathrm{KO}$ and WT samples (Figures 3.5, 3.6; outer nuclear layer/total: WT: $0.321 \pm 0.024, \mathrm{n}=4$; KO: $0.324 \pm 0.024, \mathrm{n}=5$ ), indicative of a normal overall development of the retina in the absence of Neuroligin 2. Furthermore, the number of photoreceptors, as determined on Nissl-stained semithin sections, was comparable in WT and KO (WT: $191 \pm 17$ per $100 \mu \mathrm{m}$ length, $\mathrm{n}=4$; KO: $197 \pm 13$ per $100 \mu \mathrm{m}$ length, $\mathrm{n}=5$ ). The integrity of photoreceptor connectivity at the OPL was examined by labeling for Bassoon (a marker of rod and cone ribbon synapses; Brandstatter et al., 1999), peanut agglutinin (PNA; a specific marker for cone pedicles; Haverkamp et al., 2001), Goo (a specific marker for rod and ON-cone bipolar cells; Vardi, 1998) and Calbindin (a marker of horizontal cells; Peichl and Gonzalez-Soriano, 1994) (Figure 3.5A). Taken together, these markers indicated that the number and spread of photoreceptor synapses was normal and that horizontal and bipolar cell arborizations were intact in the Neuroligin $2 \mathrm{KO}$ retina. Thus, the OPL organization is preserved in the absence of Neuroligin 2.

Rod bipolar cells, which are central to dim-light (scotopic) vision, were identified by labeling with PKC $\alpha$ (Haverkamp and Wassle, 2000), and no changes were observed in their number or lamination in the Neuroligin 2-deficient retina (Figure 3.5B). Similarly, at the IPL, conventional and bipolar cell ribbon synapses (labeled respectively with Synapsin (Mandell et al., 1992), and VGLUT1 (Johnson et al., 2003)) were present at normal densities (Figure $3.5 \mathrm{C})$.

Finally, wide-field GABAergic and small-field glycinergic amacrine cells, responsible for inhibition at the IPL (Pourcho and Goebel, 1985; Pow and Hendrickson, 1999), were stained for glutamic acid decarboxylase (GAD) 65 and 67 (Vardi and Auerbach, 1995; Haverkamp and Wassle, 2000) and the glycine transporter GlyT1 (Zafra et al., 1995; Menger et al., 1998; Pow and Hendrickson, 1999), respectively. The arborization and lamination profile of GABAergic cells was unaltered in the Neuroligin $2 \mathrm{KO}$ retina (GAD 65, Figure 3.5D; GAD 67 showed similar results). In contrast, fluorescence intensities for GlyT1, which can be reliably compared between samples in the chosen experimental paradigm (Greferath et al., 1995), were higher throughout all KO samples observed (Figures 3.5D, 3.6C), and distributed over a larger surface of the IPL in the KO as compared to control (WT: $24.84 \pm 4.8 \mu \mathrm{m}^{2} / 100$ 
$\left.\mu \mathrm{m}^{2}, \mathrm{n}=5 ; \mathrm{KO}: 47.55 \pm 6.18 \mu \mathrm{m}^{2} / 100 \mu \mathrm{m}^{2}, \mathrm{n}=5 ; \mathrm{p}=0.023\right)$, indicating elevated levels of GlyT1 in the Neuroligin $2 \mathrm{KO}$ retina.

Thus, the absence of Neuroligin 2 did not compromise the accurate formation of the basic cellular and synaptic architecture of the adult retina. However, it yielded altered density and distribution of the glycinergic amacrine cell marker GlyT1. This finding was unexpected given the co-partitioning of Neuroligin 2 with $\mathrm{GABA}_{\mathrm{A}}$ (and not glycine) receptors, and might reflect subtle homeostatic rearrangements within the retinal circuitry. To investigate further the inhibitory postsynaptic compartment, wild-type and Neuroligin 2-deficient retinae were labeled for GlyR and GABA receptor subunits.

A

B
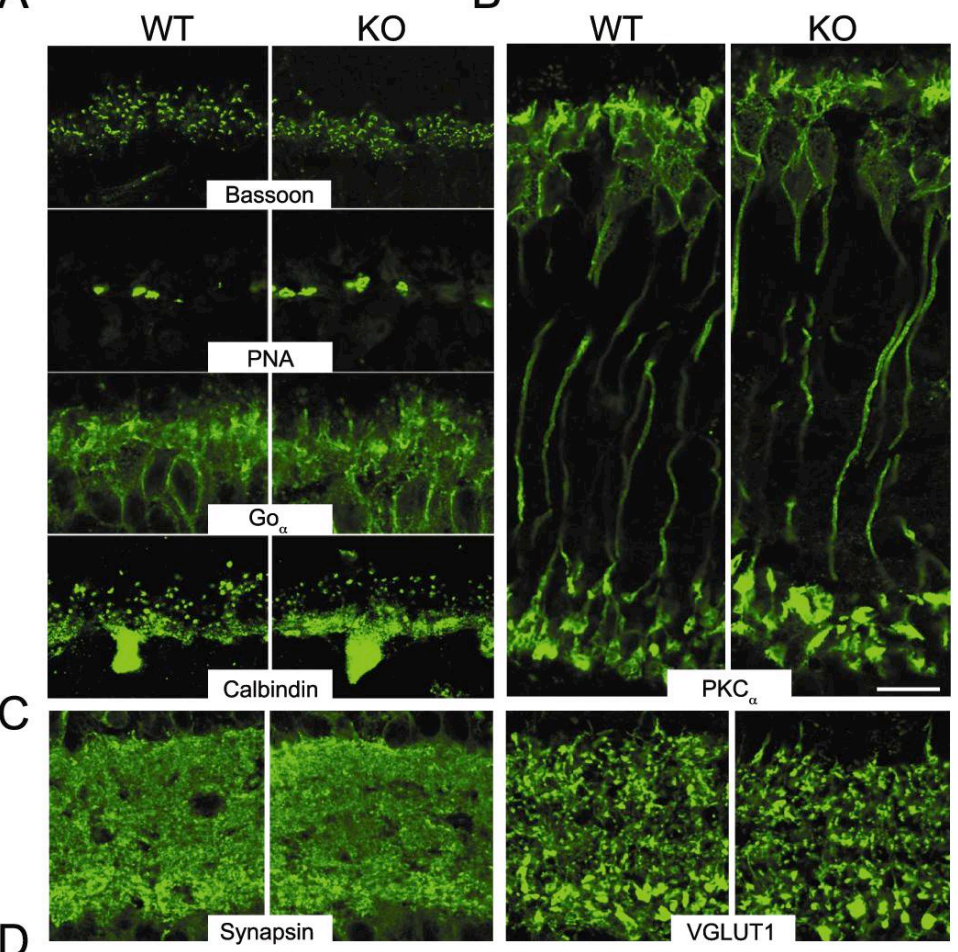

Figure 3.5: Organization of the Neuroligin 2-deficient retina

The architecture at the OPL was preserved in the absence of Neuroligin 2 (KO), as demonstrated by labeling wild-type (WT) and KO sections with Bassoon (ribbon synapses), PNA (cone pedicles) Goo (ON-bipolar cell dendrites) and Calbindin (horizontal cells) (A).

The rod bipolar cell population (labeled with $\mathrm{PKC} \alpha$ ) was also intact upon Neuroligin 2 deletion (B).

At the IPL, conventional (labeled with Synapsin) and ribbon synapses

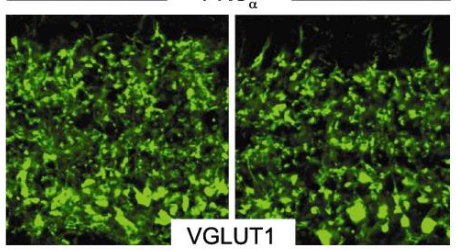
(detected by VGLUT1) were observed in normal density, in the Neuroligin 2deficient retinae $(\mathbf{C})$.

Finally, GABA- and glycinergic amacrine cell populations were labeled
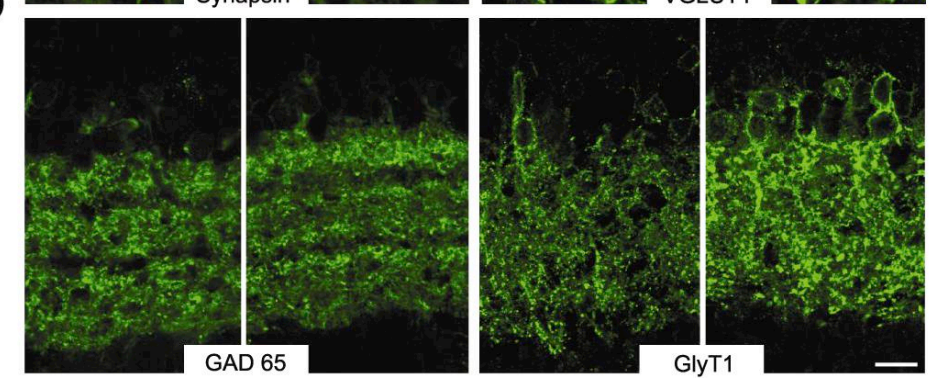
with GAD-65 and GlyT1 respectively (D). Labeling for GlyT1 was significantly stronger in the Neuroligin 2-deficient retina samples. Further, the area covered by the glycinergic amacrine cell processes was also significantly increased (D) in the KOs. Scale bar $=10 \mu \mathrm{m}$.

Adapted from Hoon et al., 2009. 


\subsubsection{Lack of Neuroligin 2 results in a specific reduction of $\mathrm{GABA}_{\mathrm{A}}$ receptor clustering}

The distribution patterns of Gephyrin, GABA, and glycine receptors were compared in Neuroligin $2 \mathrm{KO}$ and WT retinae by quantifying both numbers and fluorescence intensities of the immunolabeled puncta (Figure 3.6A-C).

As a vast majority of Neuroligin 2 puncta was found to colocalize with the ubiquitous $\mathrm{GABA}_{\mathrm{A}}$ receptor subunit $\mathrm{GABA}_{\mathrm{A}} \gamma 2$ (Figure 3.2), the distribution of this subunit was first examined in Neuroligin 2-deficient retinae. The number of $\mathrm{GABA}_{\mathrm{A}} \gamma 2$-immunoreactive puncta was dramatically reduced in the absence of Neuroligin 2 (Figure 3.6A, B). As the $\mathrm{GABA}_{\mathrm{A}} \gamma 2$ subunit has been shown to confer $\mathrm{GABA}_{\mathrm{A}}$ receptors their functional properties (Essrich et al., 1998; Moss and Smart, 2001; Alldred et al., 2005), this result indicates a reduced number of functional $\mathrm{GABA}_{\mathrm{A}}$ receptors in the Neuroligin 2-deficient retina. $\mathrm{GABA}_{\mathrm{A}} \alpha 3$ clusters were also significantly reduced in numbers (Figure 3.6A, B). In contrast, $\mathrm{GABA}_{\mathrm{A}} \alpha 1$ clusters were detected in normal numbers (Figure 3.6A, B), but were markedly dimmer in the $\mathrm{KO}$ as compared to the WT retina (Figure 3.6C). The average number of $\mathrm{GABA}_{\mathrm{C}}$ receptor puncta also seemed to be lower in $\mathrm{KO}$ retina (Figure 3.6B), but the difference did not reach statistical significance. In summary, Neuroligin 2 colocalized with $\mathrm{GABA}_{\mathrm{A}}$ receptors in WT retina, and Neuroligin 2 deficiency decreased dramatically, the abundance of specific populations of $\mathrm{GABA}_{\mathrm{A}}$ receptors.

Next, the distribution of Gephyrin, which only partially colocalized with Neuroligin 2 in WT retina (Figure 3.2), was examined. Neither the number, distribution or intensity of Gephyrin clusters varied noticeably in the Neuroligin 2 mutant retina (Figure 3.6A-C), indicating that the specific $\mathrm{GABA}_{\mathrm{A}}$ receptor clusters that are lost concomitant to Neuroligin 2 deletion might rely on a gephyrin-independent mechanism of clustering (discussed in 1.2.3).

Although a minority of Neuroligin 2 puncta associated with GlyR clusters (Figure 3.2), a lack of Neuroligin 2 led to an upregulation of GlyT1 (Figure 3.5, 3.6). This prompted the analysis of GlyR containing postsynapses in the Neuroligin 2-deficient retinae. GlyR clusters, however, did not vary in number, fluorescence intensity or distribution (Figure 3.6A-C), subsequent to Neuroligin 2 deletion. This demonstrates that Neuroligin 2 is required for the integrity of $\mathrm{GABA}_{\mathrm{A}}$ but not glycine receptors, consistent with its distribution. The next step was then to study whether this impairment at the GABAergic postsynaptic compartment in the Neuroligin 2-deficient retina would translate into alterations of retinal output. Hence, the coding of light by retinal ganglion cells was examined by multielectrode arrays. 


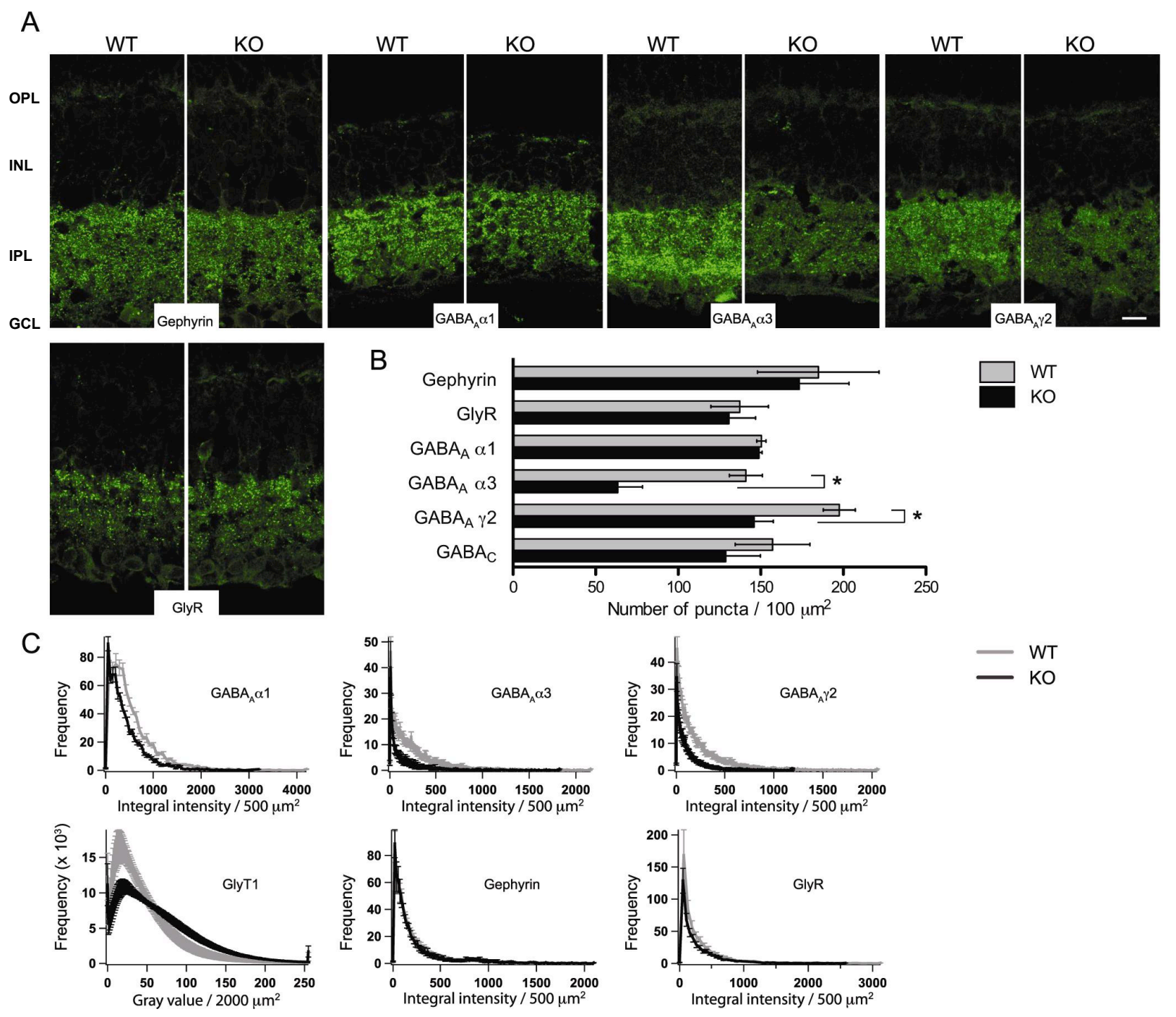

Figure 3.6: Specific impairment of $\mathbf{G A B A}_{\mathrm{A}}$ receptor clustering subsequent to Neuroligin 2 deletion

Representative confocal micrographs (A), numbers (B) and fluorescence intensity profiles $(\mathbf{C})$ of puncta immunoreactive for Gephyrin, Glycine receptors (GlyR), GABA $A_{A}$ receptor $\alpha 1, \alpha 3$ or $\gamma 2$ subunits in the IPL of wild-type (WT) and Neuroligin 2-deficient (KO) retinae ( $\mathrm{n}>6$ mice). While Gephyrin and GlyR clusters distributed normally, $\mathrm{GABA}_{\mathrm{A}} \gamma 2$ and $\mathrm{GABA}_{\mathrm{A}} \alpha 3$ immunolabeled puncta were far less abundant in the $\mathrm{KO}$ retina $(\mathbf{A}, \mathbf{B}) . \mathrm{GABA}_{\mathrm{A}} \alpha 1$ clusters appeared fainter, albeit present in normal amount (B, C). Of note, GlyT1 labeling was dimmer in the WT as compared to the Neuroligin $2 \mathrm{KO}$, as represented by the high occurrence of low-intensity values (see also Fig. 3.5D). OPL, outer plexiform layer; INL, inner nuclear layer; IPL, inner plexiform layer; GCL, ganglion cell layer

Scale bar $=10 \mu \mathrm{m}$.

Adapted from Hoon et al., 2009. 


\subsubsection{Ganglion cells in the Neuroligin 2-deficient retina show increased baseline firing rate and impaired responses to light onset}

To investigate whether the impaired $\mathrm{GABA}_{\mathrm{A}}$ receptor clustering in the Neuroligin 2-deficient animals would have functional consequences on retinal processing of visual stimuli, ganglion cell spiking activity was analyzed. Action potential firing of individual retinal ganglion cells were recorded in retina patches using multielectrode arrays (MEA; Sakai and Naka, 1992; Berry et al., 1997; Chichilnisky, 2001; Chichilnisky and Kalmar, 2002; Fairhall et al., 2006). These recordings were carried out in collaboration with Dr. Bjoern Falkenburger, at the Dept of Neurodegeneration and Restorative Research, University of Göttingen.

Neurons were classified as ON, OFF, or ON-OFF cells based on the pattern of their response to a $1 \mathrm{sec}$ light stimulus (Figure 3.7A, WT, $\mathrm{n}=350$ cells; $\mathrm{KO}, \mathrm{n}=366$ cells). No significant differences in the relative occurrences of $\mathrm{ON}, \mathrm{OFF}$, and $\mathrm{ON}-\mathrm{OFF}$ ganglion cells were observed between retina patches from WT and Neuroligin 2-deficient animals (Figure 3.7B), indicating that Neuroligin 2 deficiency did not lead to the loss of either of these pathways. This results adds functional evidence to the observation that the core architecture and main signal transmission pathway is unaffected by Neuroligin 2 deletion (Figure 3.5). Interestingly, however, the baseline activity of ganglion cells, measured as the number of action potentials per second during the second preceding light onset, was significantly increased in Neuroligin 2-deficient animals (Figure 3.7C). The baseline firing rate of a recorded ganglion cell was next, scaled to the total number of spikes recorded from a given cell (Figure 3.7D), and when analyzed for the three groups (ON, OFF and ON-OFF) of ganglion cells, this parameter was also significantly increased in the Neuroligin 2-deficient ganglion cells, indicating that in the absence of Neuroligin 2 the retinal ganglion cells allocate a greater proportion of their spikes for baseline, i.e. non-stimulus related, activity. The elevated baseline was most pronounced for the ON-subtype of ganglion cells (Figure 3.7C, D), and when the entire population of ganglion cells was pooled, the increase in baseline spiking activity remained significant in the $\mathrm{KO}$ as compared to the WT (Figure 3.7E). The observation that the Neuroligin 2-deficient ganglion cells display increased baseline firing rate relates to an impaired surround inhibition at the IPL and is consistent with the GABAergic deficit observed morphologically.

While the Neuroligin 2-deficient ganglion cells exhibit increased baseline (in the absence of the light stimulus) firing, the maximum absolute firing rate during light stimulation (Figure 3.7F, I), for both the ON and the OFF responses, was similar between WT and KO ganglion cells. For neurons receiving information from retinal ganglion cells, the distribution of spikes over time, and in relation to the stimulus, is more important than the overall number of spikes 
in a given time period. Therefore, each light response was scaled to the total number of spikes recorded from a given cell. With this normalization, the amplitude of responses to light onset was significantly reduced in $\mathrm{KO}$ cells (Figure $3.7 \mathrm{G}$ ), indicating that a reduced proportion of spikes is coding for light. Again, this effect was most pronounced in ON-type ganglion cells (Figure $3.7 \mathrm{G}, \mathrm{H}$ ). On the other hand, the response amplitude to light offset was not significantly altered in $\mathrm{KO}$ cells (Figure 3.7J).

Taken together, these observations indicate that in retinal ganglion cells from Neuroligin 2deficient animals, an increased baseline activity impairs the coding of light stimuli. Therefore, a loss of Neuroligin 2 has a deleterious effect on $\mathrm{GABA}_{\mathrm{A}}$ receptor clustering at the IPL of the retina, which leads to functional consequences by impairing the stimulus coding efficacy and hence information processing by the output stations (the ganglion cells) of the retina.

A

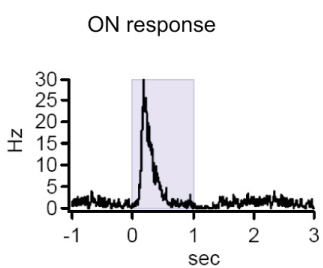

C

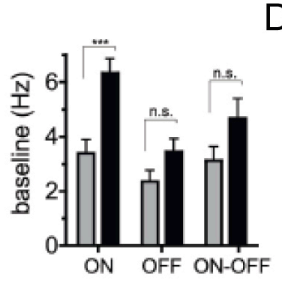

$\mathrm{F}$

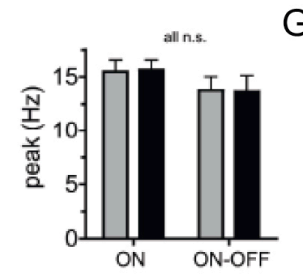

G

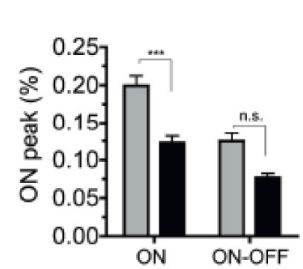

$\mathrm{H}$

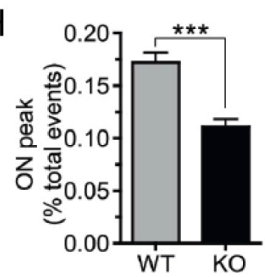

I
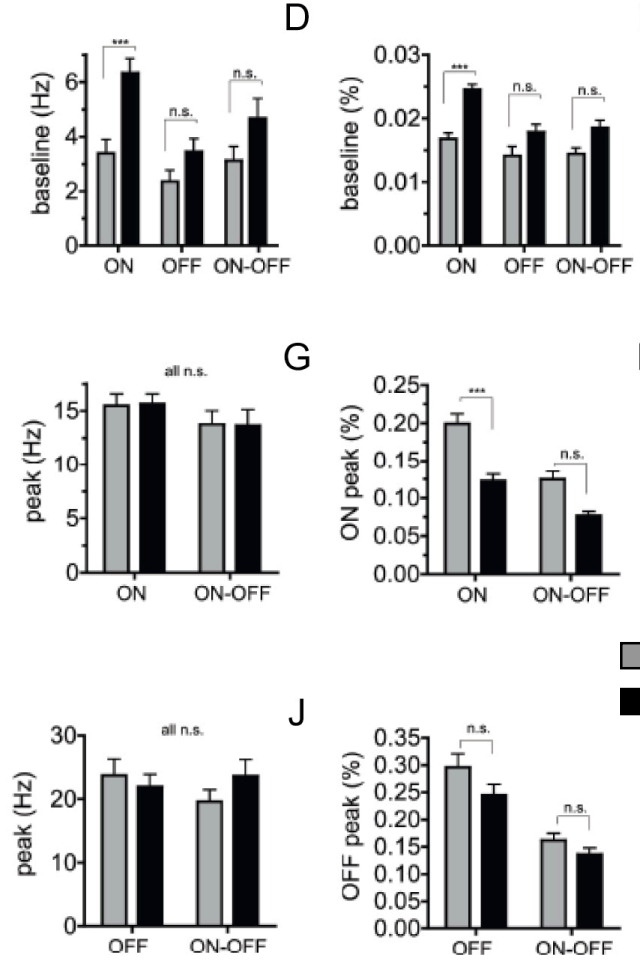

E

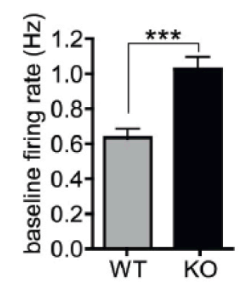

$\square$ wT

KO

Adapted from Hoon et al., 2009
B
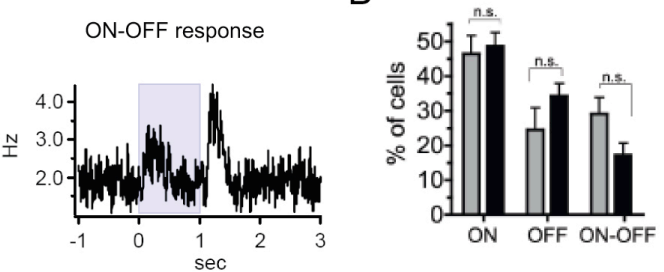

Figure 3.7: Light responses of Neuroligin 2-deficient ganglion cells

Responses of ganglion cells to light onset (ON), offset (OFF) and both onset-offset (ON-OFF) (A). Relative occurrence of the three cell types in wild-type (WT) and Neuroligin 2deficient (KO) retinae (B). Baseline firing was significantly increased in Neuroligin $2 \mathrm{KO}$ ganglion cells $(\mathbf{C})$. Baseline activity expressed as a percentage of all spikes (D) and baseline activity pooled for the different types of ganglion cells $(\mathbf{E})$, reflected this difference. Maximal ON (F) and OFF (I) response peaks remained unaltered in the absence of Neuroligin 2; however, the responses normalized to the total number of recorded spikes $(\mathbf{G}, \mathbf{J})$, displayed a significant reduction in the proportion of spikes coding for light onset (G). Normalized ON responses pooled for the different ganglion subtypes (H) also displayed the impairment. 


\subsubsection{GABA receptor clustering is disrupted at the CA1 region of the hippocampus, subsequent to Neuroligin 2 deletion}

In order to investigate whether the impairment in $\mathrm{GABA}_{\mathrm{A}}$ receptor clustering may also be observed in other areas of the CNS, the CA1 region of the hippocampus was labeled for $\mathrm{GABA}_{\mathrm{A}} \gamma 2$ receptor subunit, the inhibitory scaffold protein Gephyrin and the inhibitory presynaptic marker VIAAT (Figure 3.8). Principal excitatory neurons in this region are the pyramidal cells; their soma are located in the Stratum pyramidale, with their main dendrites projecting into the Stratum radiatum, which essentially receives information from the CA3 region of the hippocampus via schaffer collaterals, but also from the entorhinal cortex and the nucleus reuniens of the thalamus. In turn, the axons of the CA1 pyramidal cells project to the subiculum and the deep layers of the entorhinal cortex (Amaral and Lavenex, 2006).

Interestingly, there was a lamina-specific loss of $\mathrm{GABA}_{\mathrm{A}}$ receptor clustering in the hippocampi from Neuroligin 2-deficient animals. The number of $\mathrm{GABA}_{\mathrm{A}} \gamma 2$ receptor clusters was specifically reduced in the Stratum pyramidale $\left(\mathrm{WT}_{\text {mean }}=48.50 \pm 3.97\right.$ puncta $/ 100 \mu \mathrm{m}^{2}$; $\mathrm{KO}_{\text {mean }}=29.44 \pm 1.34$ puncta $/ 100 \mu \mathrm{m}^{2} ; \mathrm{n}=3$ pairs; $\left.\mathrm{p}=0.0453\right)$ but not in the Stratum radiatum $\left(\mathrm{WT}_{\text {mean }}=53.11 \pm 12.62\right.$ puncta $/ 100 \mu \mathrm{m}^{2} ; \mathrm{KO}_{\text {mean }}=51.52 \pm 4.23$ puncta/ $100 \mu \mathrm{m}^{2}$; $\mathrm{n}=3$ pairs; $\mathrm{p}=0.9159$ ) of Neuroligin 2-deficient CA1 region (Figure 3.8A, B). Furthermore, this reduction in $\mathrm{GABA}_{\mathrm{A}}$ receptor clustering was paralleled with a similar, lamina-specific reduction in the number of Gephyrin clusters (Stratum pyramidale: $\mathrm{WT}_{\text {mean }}=17.96 \pm 2.429$ puncta $/ 100 \mu \mathrm{m}^{2} ; \mathrm{KO}_{\text {mean }}=5.91 \pm 1.73$ puncta/ $100 \mu \mathrm{m}^{2} ; \mathrm{n}=3$ pairs; $\mathrm{p}=0.0273$; Stratum radiatum: $\mathrm{WT}_{\text {mean }}=29.55 \pm 4.50$ puncta $/ 100 \mu \mathrm{m}^{2} ; \mathrm{KO}_{\text {mean }}=29.46 \pm 8.32$ puncta $/ 100 \mu \mathrm{m}^{2} ; \mathrm{n}$ $=3$ pairs; $\mathrm{p}=0.9931$ ) (Figure 3.8C, D), demonstrating that a loss of Neuroligin 2 affects the postsynaptic clustering of $\mathrm{GABA}_{\mathrm{A}}$ receptors in this area of the CNS by a gephyrin-dependent mechanism. Importantly, there was no significant reduction observable in the number of VIAAT puncta in either of the laminae (Figure 3.8E) (Stratum pyramidale: $\mathrm{WT}_{\text {mean }}=36.51 \pm$ 2.62 puncta $/ 100 \mu \mathrm{m}^{2} ; \mathrm{KO}_{\text {mean }}=37.07 \pm 1.53$ puncta $/ 100 \mu \mathrm{m}^{2} ; \mathrm{n}=3 \mathrm{WT}, 5 \mathrm{KO} ; \mathrm{p}=0.857$ : Stratum radiatum: $\mathrm{WT}_{\text {mean }}=31.95 \pm 4.04$ puncta $/ 100 \mu \mathrm{m}^{2} ; \mathrm{KO}_{\text {mean }}=29.18 \pm 2.65$ puncta/ 100 $\mu \mathrm{m}^{2} ; \mathrm{n}=3 \mathrm{WT}, 5 \mathrm{KO} ; \mathrm{p}=0.58$ ), indicating that the number of inhibitory synapse subsequent to Neuroligin 2 deletion is unchanged. This corroborates previous observations made in the immature brain and in mature cultured neurons that a loss of Neuroligins affects the maturation of the postsynaptic compartment without a change in synapse number (Varoqueaux et al., 2006). 
Taken together, the deficits in inhibitory postsynaptic components observed at the CA1 region, correlate with the results from the retina, thus validating the retina as a model system and illustrating that Neuroligin 2 is essential for the proper clustering of postsynaptic GABA receptors in the CNS.
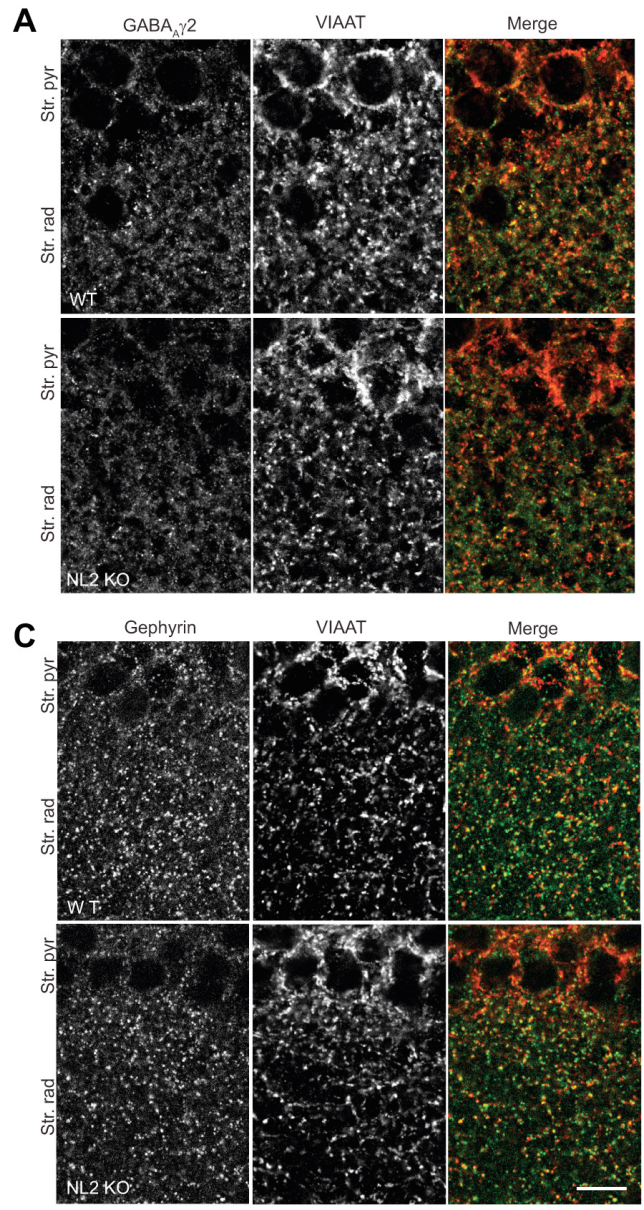

B

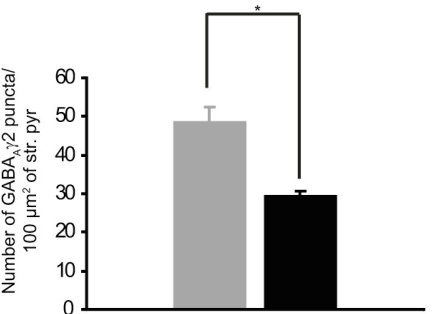

D

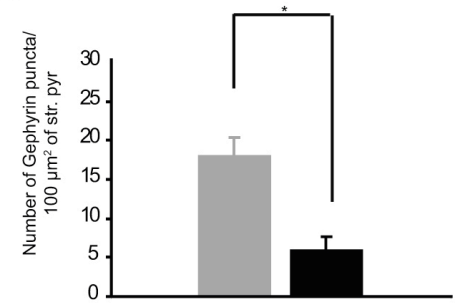

E

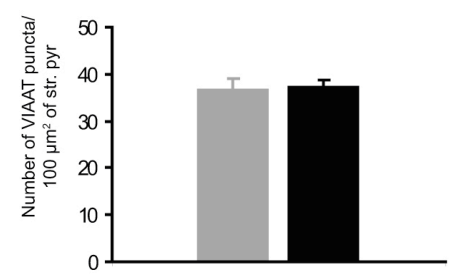

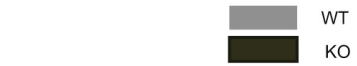
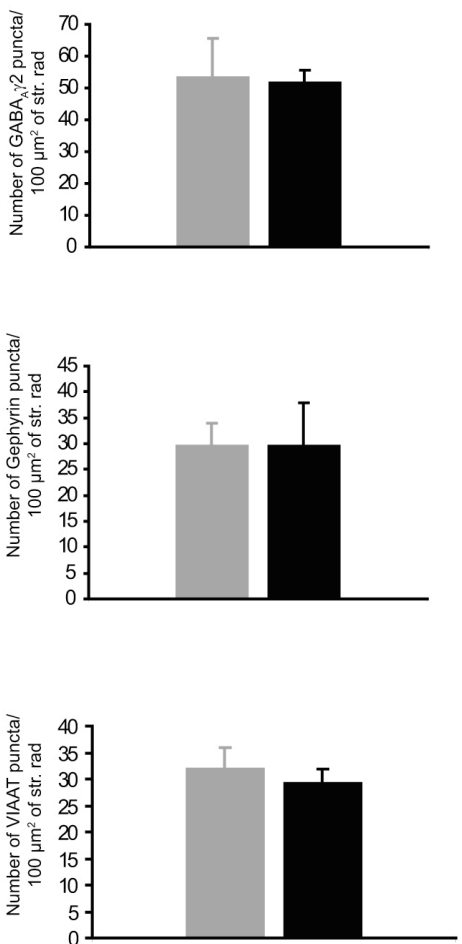

Figure 3.8: Lamina specific loss of $\mathrm{GABA}_{A}$ receptor clustering in the CA1 region of the Neuroligin 2-deficient mouse

Representative labelings of $\mathrm{GABA}_{\mathrm{A}} \gamma 2 / \operatorname{VIAAT}(\mathbf{A})$ and Gephyrin/VIAAT (C) in the CA1 region of wild-type (WT) and Neuroligin 2-deficient (KO) animals. There is a specific loss in the number of $\mathrm{GABA}_{\mathrm{A}} \gamma 2$ (B) and Gephyrin (D) clusters at the Stratum pyramidale (Str. pyr), but not at the Stratum radiatum (Str. rad). Further, the number of inhibitory synapses, estimated by VIAAT labelings, remains unaltered at both layers $(\mathbf{E})$.

Scale bar $=25 \mu \mathrm{m}$.

Adapted from Poulopoulos et al., 2009. 


\subsection{Functional characterization of Neuroligin 4}

Of all the Neuroligins, Neuroligin 4 has drawn most attention, as several mutations in this protein have been found in patients with ASDs (discussed in 1.1.6). However, no study as yet has described its localization or function at CNS synapses. Therefore, the next part of the project investigated the distribution of Neuroligin 4 at retinal synapses and this was followed by an investigation of retinal architecture and function in the Neuroligin 4-deficient mouse. Interestingly, these mice display deficits in social interaction and communication reminiscent of ASDs in humans (Jamain et al., 2008), and have thus been introduced as an animal model for autism. Accordingly, analysis of retinal function in the Neuroligin 4-deficient animals would help understand the underlying mechanism of the visual alterations observed in a subset of autistic patients (reviewed in Dakin and Frith, 2005; Behrmann et al., 2006).

\subsubsection{Neuroligin 4 is preferentially localized at glycinergic postsynapses}

An isoform-specific antibody (Jamain et al., 2008) was used to study the distribution of Neuroligin 4 in the mouse retina. Neuroligin 4 immunopositive puncta were localized exclusively at the IPL and were concentrated at the outer (OFF) sublaminae with a rather sparse distribution through the rest of the IPL (Figure 3.1C). This pattern of Neuroligin 4 labeling at the IPL was reminiscent of the distribution of GlyRs in the retina (Wassle et al., 1998). Upon colabeling with mAb4a, the antibody reported to recognize all GlyR subunits, the vast majority of Neuroligin 4 puncta were indeed located at glycinergic postsynapses (Figure 3.9A). When quantified, $73.5 \pm 4.2 \%$ of Neuroligin 4 puncta colocalized with GlyR subunits (Figure 3.9E). Thus, in the retina, Neuroligin 4 is associated specifically with glycinergic inhibitory postsynapses and interestingly, is the first Neuroligin isoform to display such selectivity towards glycinergic postsynapses.

Glycinergic neurotransmission plays an essential role for information processing at the retina (Marc, 1989; Vaney, 1990; Wassle and Boycott, 1991), since activity at the IPL relies on equal populations of inhibitory GABA- and glycinergic amacrine cells (Wassle et al., 1998). In particular, the principal rod (dim light) pathway relies on the small-field AII glycinergic amacrine cells for signal propagation to the ganglion cells (discussed in 1.2.1.2). Hence, it was important to estimate the proportion of glycinergic synapses in the retina that associate with Neuroligin 4. Upon quantifications, only $21.3 \pm 3.46 \%$ of GlyR clusters in the retina, associated with Neuroligin 4, indicating that Neuroligin 4 only equips a subset of glycinergic postsynapses at the IPL of the retina. 
Since GlyR $\alpha 1$ immunoreactivity is also enriched at the OFF sublamina of the IPL (SassoePognetto et al., 1994; Wassle et al., 1998), a double labeling was carried out for Neuroligin 4 and GlyR $\alpha 1$. While a prominent fraction of Neuroligin 4 puncta colocalized with GlyR $\alpha 1$ (Figure 3.9B), it also associated with other GlyR postsynapses ( $\alpha 2, \alpha 3$; data not shown). These results indicate that Neuroligin 4 expression relates, in part, to the entire glycinergic circuit.

In contrast, Neuroligin 4 puncta were absent from excitatory postsynaptic clusters, as estimated by co-labeling with PSD-95 (Figure 3.9C). As illustrated Neuroligin 4 clusters did not colocalize with PSD-95 puncta and upon quantifications, only $1.9 \pm 0.8 \%$ of Neuroligin 4 puncta associated with PSD-95 positive clusters (Figure 3.9E).

Additionally to further refine the localization of Neuroligin 4 at inhibitory synapses, postembedding immunogold labelings were carried out, where Neuroligin 4-positive gold particles were observed selectively at symmetric synapses of the IPL (Figure 3.9D).

Furthermore, a small fraction of Neuroligin 4 puncta $(18.4 \pm 6 \%)$ colocalized with $\mathrm{GABA}_{\mathrm{A}} \gamma 2$ receptor clusters (Figure 3.9E) As $\mathrm{GABA}_{\mathrm{A}} \gamma_{2}$ is the ubiquitous $\mathrm{GABA}_{\mathrm{A}}$ receptor subunit distributed in the retina (Wassle et al., 1998), one can assume that a very small fraction of Neuroligin 4 is also localized at GABAergic postsynapses. Clearly, the majority of Neuroligin 4 is clustered at glycinergic postsynapses in the retina. 
A
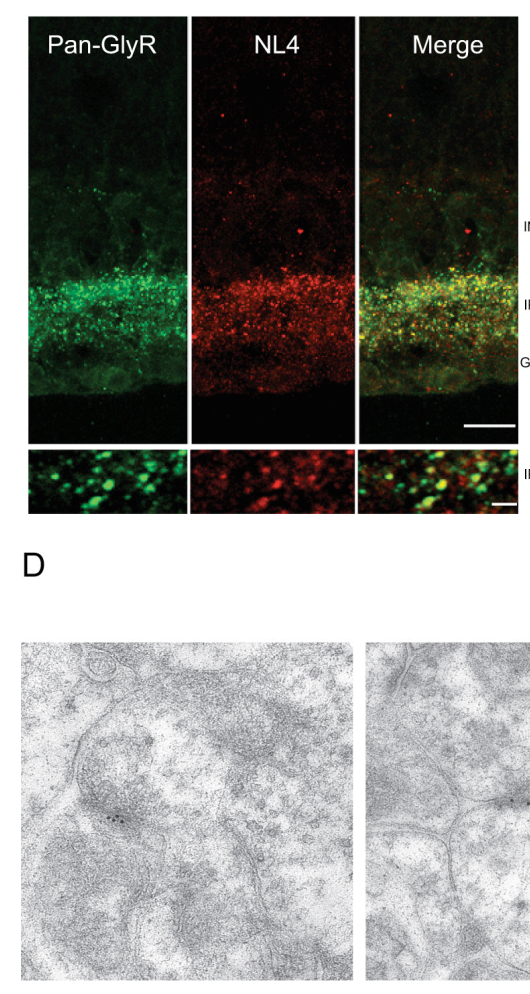

B

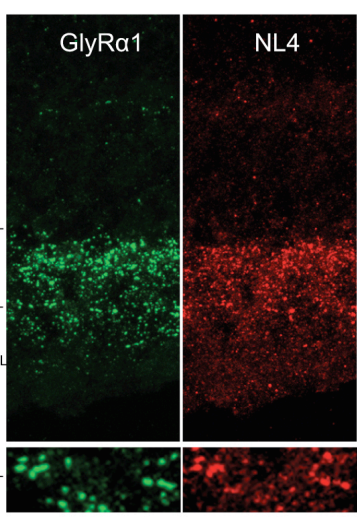

C

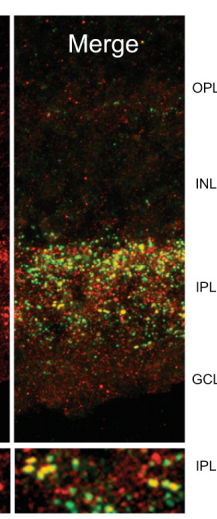

$\mathrm{E}$

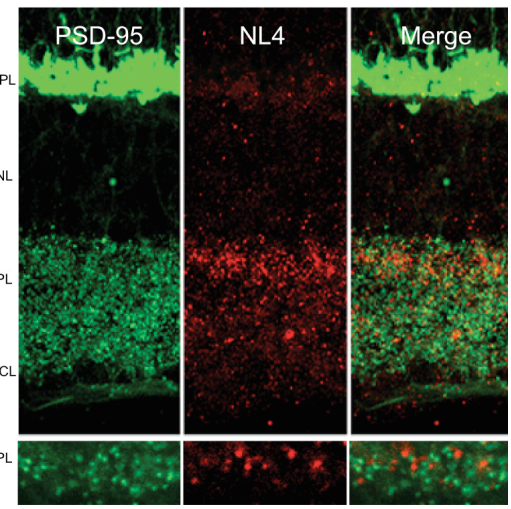

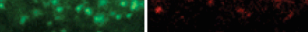

D

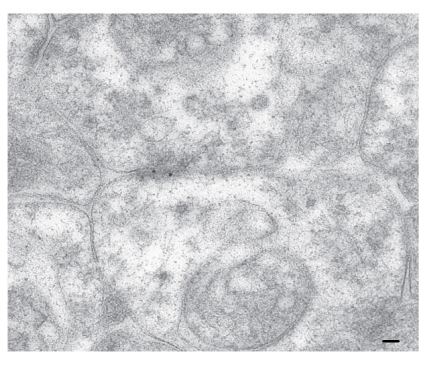

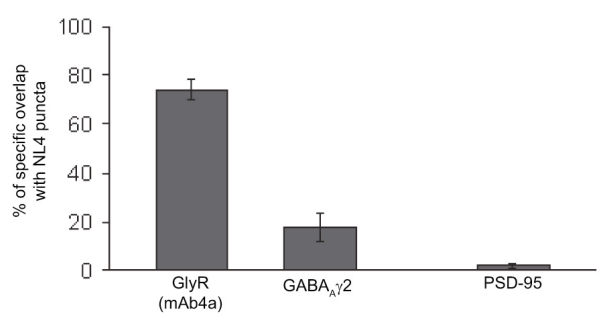

Figure 3.9: Neuroligin 4 is localized specifically at glycinergic postsynapses of the retina

Immunolabeling for Neuroligin 4 (NL4) together with a pan-GlyR antibody (mAb4a) revealed extensive colocalization of Neuroligin 4 puncta with GlyR clusters (A). In addition, co-labeling of Neuroligin 4 with a subset of GlyR synapses (GlyR $\alpha 1)$ also demonstrated a significant overlap (B). On the contrary, Neuroligin 4 puncta did not associate with PSD-95 labeled excitatory postsynaptic clusters $(\mathbf{C})$.

Postembedding immunolabeling revealed the localization of Neuroligin 4-specific gold particles at symmetric (inhibitory) synapses of the IPL (D).

Quantification of the colocalization between Neuroligin 4 and the postsynaptic markers: GlyR, $\mathrm{GABA}_{\mathrm{A}} \gamma 2$ and PSD-95 (E). OPL, outer plexiform layer; INL, inner nuclear layer; IPL, inner plexiform layer; GCL, ganglion cell layer. Scale bars = overview: $10 \mu \mathrm{m}$, detail: $1 \mu \mathrm{m}$. For EM panel scale bar $=25 \mathrm{~nm}$.

\subsubsection{Loss of Neuroligin 4 does not disrupt the synaptic network of the retina, but for a}

\section{slight reduction in a GlyR subset}

To investigate the architecture of the retina subsequent to Neuroligin 4 deletion, immunolabelings utilizing diverse cellular and synaptic markers were carried out in pairs of wild-type (WT) and Neuroligin 4-deficient retinae (Figure 3.10). Inhibitory (VIAAT) and excitatory (VGLUT1) (Johnson et al., 2003) presynapses, inhibitory (Gephyrin) (SassoePognetto and Wassle, 1997) and excitatory (PSD-95) (Koulen et al., 1998b) postsynapses, GABAergic amacrine cells (as visualized by GAD65 and GAD 67) (Vardi and Auerbach, 
1995; Haverkamp and Wassle, 2000), rod bipolar cells (labeled with PKC $\alpha$ ) (Haverkamp and Wassle, 2000), glycinergic amacrine cells (labeled by glycine transporter GlyT1) (Zafra et al., 1995; Menger et al., 1998; Pow and Hendrickson, 1999), and $\mathrm{GABA}_{\mathrm{A}}$ receptor populations (distinct subclasses labeled with $\mathrm{GABA}_{A} \gamma 2, \mathrm{GABA}_{\mathrm{A}} \alpha 1, \mathrm{GABA}_{\mathrm{A}} \alpha 2$, and $\mathrm{GABA}_{\mathrm{A}} \alpha 3$ subunit specific antibodies) (Greferath et al., 1995) all seemed preserved in density and organization in the Neuroligin $4 \mathrm{KO}$ retinae as compared to WT (Figure 3.10). Therefore, the main excitatory transmission pathway and the GABAergic circuitry of the retina seem to develop normally in the absence of Neuroligin 4.
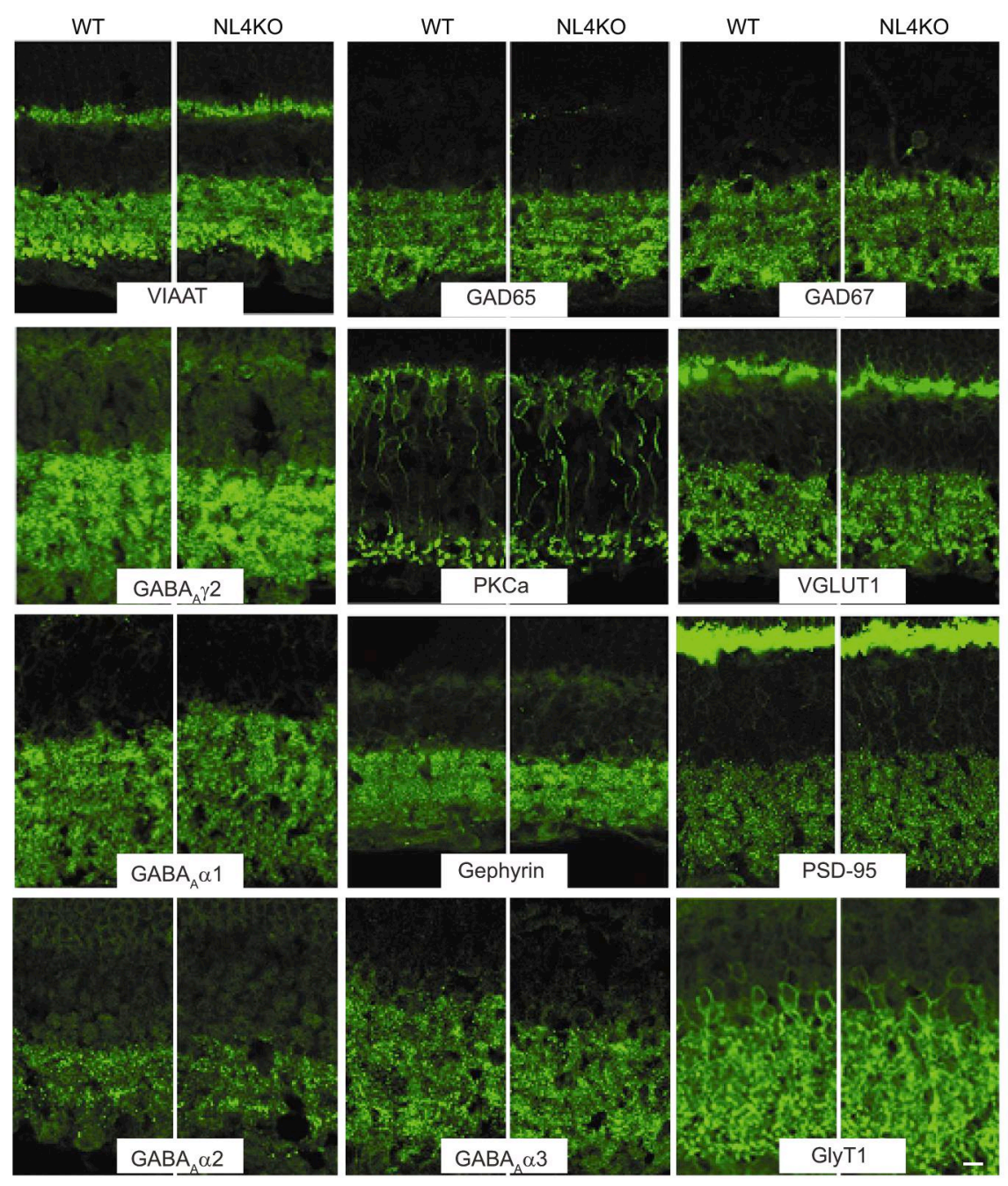

Figure 3.10: Structural organization of the Neuroligin 4-deficient retina

Selective markers were used to assess the integrity of the retinal circuit, subsequent to a loss of Neuroligin 4. Pairs of wild-type (WT) and Neuroligin 4-deficient (NL4KO) retinae were labeled for inhibitory (VIAAT) and excitatory (VGLUT1) presynapses, inhibitory (Gephyrin) and excitatory (PSD-95) postsynapses, GABAergic (labeled by GAD65/67) and glycinergic amacrine cells (GlyT1), rod bipolar cells (PKC $\alpha$ ) and $\mathrm{GABA}_{\mathrm{A}}$ receptor subtypes $(\alpha 1, \alpha 2, \alpha 3$ and $\gamma 2)$. No structural alteration was observed in the absence of Neuroligin 4. Scale bar $=5 \mu \mathrm{m}$. 
To investigate more precisely the glycinergic postsynapses, immunolabelings were carried out for the different GlyR subunits, which represent distinct subsets of glycinergic postsynapses (Figure 3.11A). There was no dramatic reduction of GlyR clusters in the absence of Neuroligin 4 (Figure 3.11A); however a small yet significant reduction in the number of GlyR $\alpha 1$ clusters was revealed upon quantifications $\left(\mathrm{WT}_{\text {mean }}=19.08 \pm 0.77\right.$ puncta $/ 100 \mu \mathrm{m}^{2}$ of IPL, $\mathrm{KO}_{\text {mean }}=15.88 \pm 0.59$ puncta $/ 100 \mu \mathrm{m}^{2}$ of IPL, $\mathrm{n}=7$ pairs, $\mathrm{p}=0.0067$ ) (Figure 3.11B). Thus, the absence of Neuroligin 4 did not lead to a dramatic morphological alteration of the retinal circuitry or of the synapses, except for a small but consistent reduction (about 16.7\% loss in the KO as compared to the WT) in the number of the GlyR $\alpha 1$ clusters.

The GlyR $\alpha 1$ immunoreactivity is enriched at the outer (OFF) sublamina of the IPL and represents synapses between the AII amacrine and the OFF cone bipolar cells (SassoePognetto et al., 1994). In addition, GlyR $\alpha 1$ puncta are also dispersed through the remainder of the IPL representing glycinergic synapses onto ganglion cell processes and rod bipolar cell terminals (Ivanova et al., 2006; Majumdar et al., 2007). Interestingly, the $\alpha 1$ containing GlyRs located on retinal ganglion cells mediate kinetically fast glycinergic transmission, as electrophysiological recordings from ganglion cells of the GlyR $\alpha 1 \mathrm{KO}$ mice display spontaneous inhibitory postsynaptic currents (sIPSCs) with significantly longer decay time constants (Majumdar et al., 2007). Thus, to investigate if the subtle reduction in the GlyR $\alpha 1$ clusters would entail functional consequences, glycinergic activity was recorded from Neuroligin 4-deficient ganglion cells.

A

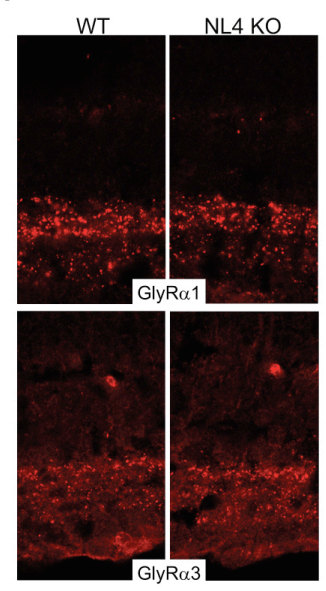

B
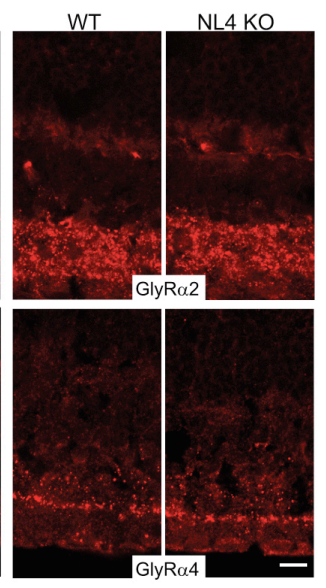

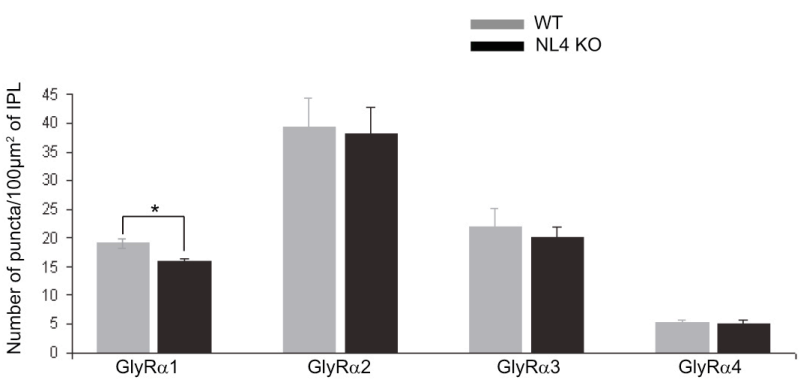

Figure 3.11: Specific loss of $\alpha 1$ containing GlyR clusters in the Neuroligin 4-deficient retina

Distinct subsets of GlyR synapses (GlyR $\alpha 1, \alpha 2, \alpha 3, \alpha 4)$ were compared in wild-type (WT) and Neuroligin 4 deficient (NL4KO) retinae (A). Upon quantification (B), a specific reduction in $\alpha 1$ containing GlyR clusters was observed in NL4 KO retinae as compared to WT ( $\mathrm{n}=7$ pairs).

Scale bar $=5 \mu \mathrm{m}$. 


\subsubsection{Glycinergic mIPSCs from Neuroligin 4-deficient retinal ganglion cells exhibit a slower decay time constant}

Higher order visual processing relies on the activity of the ganglion cells, which represent the output stations of the retina. Therefore, the next step was to examine whether the small reduction in GlyR $\alpha 1$ clusters may cause alterations in retinal signal processing. Consequently, miniature inhibitory postsynaptic currents (mIPSCs) were recorded from WT and Neuroligin 4-KO ganglion cells.

Recordings were carried out in a whole-mount preparation in order to preserve the synaptic connectivity of the IPL and to maintain the integrity of retinal ganglion cell innervation. A combination of morphological and functional parameters was imposed to ensure that mIPSCs were recorded exclusively from retinal ganglion cells and not from "displaced" amacrine cells also localized in the ganglion cell layer (see methods). An exemplar image constructed of a confocal stack from one such retinal ganglion cell is illustrated in Figure 3.12A.

While recording glycinergic mIPSCs (WT: $n=13$ animals, 25 cells; KO: $n=10$ animals, 16 cells), TTX $(1 \mu \mathrm{M})$ was applied to the bath to prevent spiking of the unclamped dendritic compartment of the retinal ganglion cells. TTX has been shown in previous studies to have no effect on the frequency of glycinergic mIPSCs as recorded from ganglion cells in rat retinal slices (Protti et al., 1997).

Glycinergic mIPSCs were recorded at a holding potential near the resting membrane potential of ganglion cells (O'Brien et al., 2003) $\left(\mathrm{V}_{\text {holding }}=-70 \mathrm{mV}\right)$, in bicarbonate buffered saline (BBS) $\left(0.5 \mathrm{mM} \mathrm{Ca}^{2+}, 1 \mathrm{mM} \mathrm{Mg}^{2+}\right)$ in the presence of NBQX $(1 \mu \mathrm{M})$ AP5 $(50 \mu \mathrm{M})$ and bicuculline $(50 \mu \mathrm{M})$. Under our recording conditions, about one-third of the ganglion cells tested exhibited glycinergic mIPSCs. The glycinergic mIPSCs from Neuroligin 4-deficient retinal ganglion cells were slightly smaller in amplitude (Fig 3.12B, C, p=0.192), unchanged in frequency (Fig 3.12C, p=0.6128), but slower in their rise time (20-80\%) (Fig 3.12C) (Although the difference was not quite significant: $\mathrm{WT}_{\text {mean }}=316.01 \pm 7.97 \mu$ sec: $\mathrm{KO}_{\text {mean }}=$ $361.94 \pm 23.34 \mu$ sec: $p=0.079$ ). However, glycinergic mIPSCs from the Neuroligin $4 \mathrm{KO}$ decayed significantly slower (with a prolonged decay time constant $\tau$ ) as compared to WT littermates (Fig 3.12B, C, $\mathrm{WT}_{\text {mean }} \tau=2.42 \pm 0.096$ msec: $\mathrm{KO}_{\text {mean }} \tau=2.87 \pm 0.15 \mathrm{msec}$ : $\mathrm{p}=0.0223$ ). Further, to study the variability of the decay kinetics among glycinergic mIPSCs, individual events (larger than $15 \mathrm{pA}$ in amplitude as smaller events could not be fit reliably) within a given cell were analyzed and the decay time constant $(\tau)$ of individual glycinergic mIPSCs estimated and plotted as a histogram. Glycinergic mIPSCs for both WT and 
Neuroligin $4 \mathrm{KO}$ were quite homogeneous with respect to their decay time constants (Figure 3.12D). Even though this analysis was performed on a 'subset' of glycinergic mIPSCs, it was sufficient to uncover a distinct rightward shift in the KO distribution for the mean $\tau$ value (Figure 3.12D; $\mathrm{WT}_{\text {mean }}: 2.50 \pm 0.043 \mathrm{msec}, \mathrm{KO}_{\text {mean }} 2.68 \pm 0.05 \mathrm{msec}$, $\mathrm{p}$ value $=0.0217$ ). This indicates that some of the fastest events (with short decay $\tau$ ) in the WT distribution were absent from the Neuroligin $4 \mathrm{KO}$ (Figure $3.12 \mathrm{D}$ ).

In contrast, the GABAergic mIPSCs (WT: $n=7$ animals, 20 cells; KO: $n=7$ animals, 22 cells) recorded from Neuroligin $4 \mathrm{KO}$ ganglion cells in the presence of strychnine (500 nM), NBQX and AP5, were unchanged in all parameters studied in comparison to WT controls (Figure $3.12 \mathrm{E}$; for all parameters $\mathrm{p}>0.3$ ). Thus, glycinergic input to retinal ganglion cells, as reflected by glycinergic mIPSCs, was specifically impaired subsequent to a loss of Neuroligin 4.

A

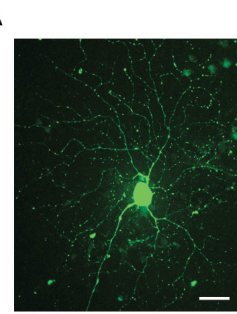

B

C
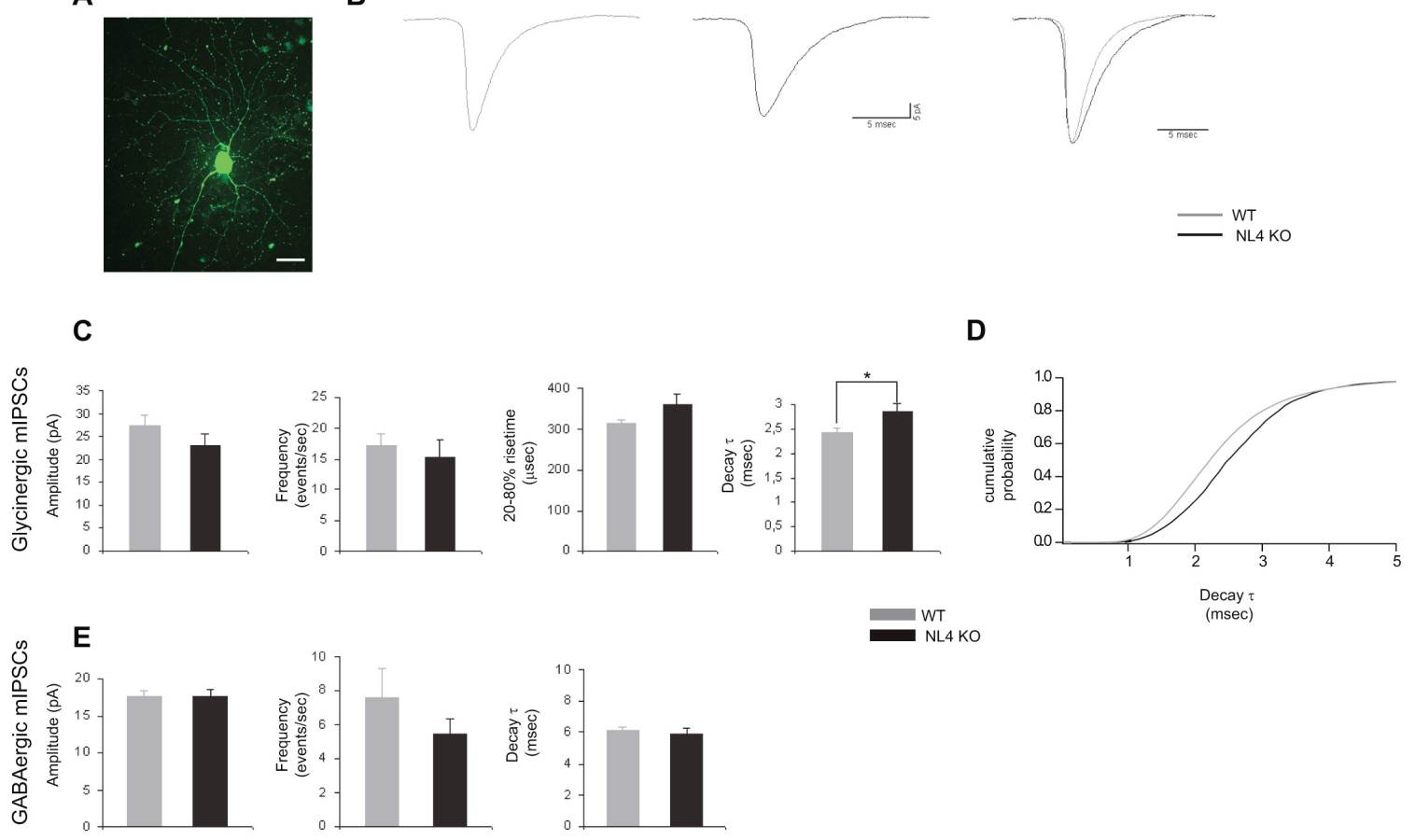

WT 


\subsubsection{Information processing in the Neuroligin 4-deficient retina}

The next question to address was whether the subtle alterations in the fast component of glycinergic inhibition at the IPL, would affect the stimulus-coding efficacy of the ganglion cells. Accordingly, we recorded stimulus-related spiking activity of Neuroligin 4-deficient retinal ganglion cells with a multi-electrode array (Figure 3.13). A 1 sec light flash applied to the retina, distinguished ganglion cells as ON, OFF, and ON-OFF (Figure 3.13A). There was no difference in the occurrence of these ganglion cell types between wild-type (WT) and Neuroligin $4 \mathrm{KO}$ retinae (Figure 3.13B). Further, there was no significant difference in the baseline firing rate, and ON/OFF peak amplitude of Neuroligin 4-deficient cells as compared to WT (Figure 3.13B). Thus, in the absence of Neuroligin 4 there is no striking alteration in ganglion cell information coding efficacy. As retinal ganglion cells form the output stations of the retina, these results would also indicate that processing at higher visual centers remains intact subsequent to Neuroligin 4 deletion.

In summary, Neuroligin 4 is localized specifically at a subset of glycinergic postsynapses in the retina, and a loss of Neuroligin 4 is associated with a subtle impairment of glycinergic processing at the IPL.

A

ON response

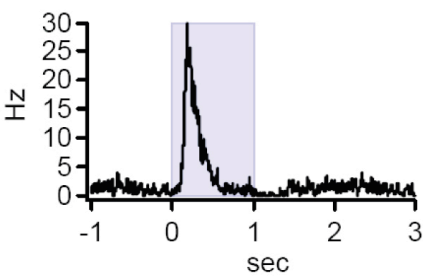

B

Occurence

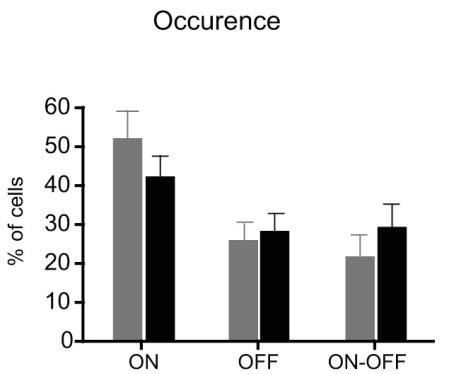

$B$
OFF response
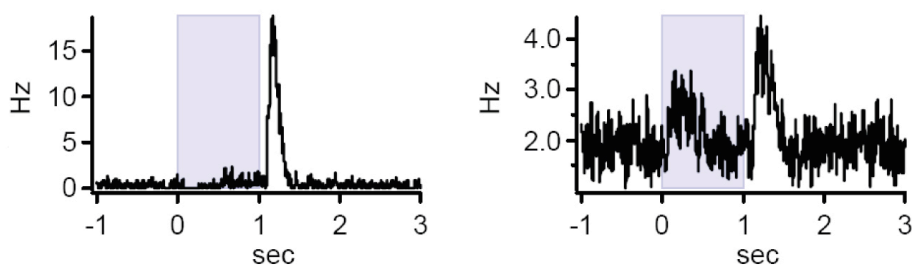

Baseline activity

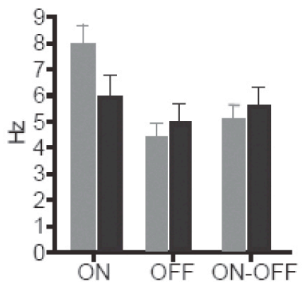

ON peak size

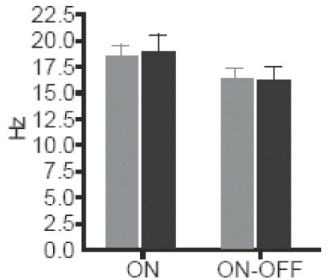

WT

NL4 KO

OFF peak size

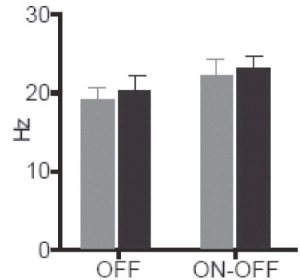

Figure 3.13: Coding of light in the Neuroligin 4-deficient ganglion cells

Representative traces from ON, OFF and ON-OFF ganglion cells firing upon the application of a $1 \mathrm{sec}$ light flash (A).

There was no alteration in the occurrence of these ganglion cell subtypes in the Neuroligin 4 KO (B). Neither was there a difference in baseline spiking or ON/OFF peak size in the Neuroligin 4-deficient ganglion cells (NL4 KO) as compared to the wild-type (WT) (B). 


\subsection{Morphological investigation of Neuroligin 3}

The final phase of the project, focused on the characterization of Neuroligin 3. Neuroligin 3 has also been implicated in autism (discussed in 1.1.6), but its distribution in the brain has not yet been thoroughly investigated. One study in hippocampal cultures, has demonstrated the presence of Neuroligin 3 at both excitatory (glutamatergic) and inhibitory (GABAergic) postsynapses (Budreck and Scheiffele, 2007). As the retina is composed of stratified synaptic connections of distinct inhibitory (subsets of GABA- and glycinergic synapses) and excitatory inputs, it served as an apt model to investigate further the distribution of Neuroligin 3 at specified CNS synapses.

\subsubsection{Neuroligin 3 is localized at GABAergic postsynapses of the retina}

Neuroligin 3 expression was abundant at the IPL of WT retinae (Figure 3.1B), and to ascertain its distribution, immunolabelings were carried out for landmark inhibitory and excitatory (preand postsynaptic) markers, together with the isoform-specific Neuroligin 3 antibody. At the IPL, double labeling of Neuroligin 3 with the excitatory postsynaptic marker PSD-95 revealed restricted colocalization with Neuroligin 3-positive puncta (Figure 3.14B). Upon quantification, $\sim 6 \%$ of Neuroligin 3 puncta were localized at excitatory postsynapses (Figure 3.14E). Thus in the retina, a majority of Neuroligin 3 puncta are devoid from excitatory postsynapses.

Conversely, Neuroligin 3 puncta associated prominently with inhibitory postsynaptic markers. Shown in Figure 3.14A is the co-distribution of Neuroligin 3 with the inhibitory postsynaptic scaffold protein Gephyrin and the ubiquitous $\mathrm{GABA}_{\mathrm{A}}$ receptor subunit $\mathrm{GABA}_{\mathrm{A}} \gamma 2$. About $55 \%$ of Neuroligin 3 colocalized with $\mathrm{GABA}_{\mathrm{A}} \gamma 2$ (Figure 3.14E), while $\sim 40 \%$ associated with Gephyrin. Interestingly, at retinal synapses, Neuroligin 3 is also distributed at glycinergic postsynapses (Figure 3.14C) and upon quantification, $\sim 40 \%$ of Neuroligin 3 puncta colocalized with GlyR clusters (Figure 3.14E). It is noteworthy, that Neuroligin 3 colocalizes with Gephyrin and GlyR clusters to a similar extent (Figure 3.14E). As Gephyrin is present at all glycinergic postsynapses of the retina, as well as a subset of $\mathrm{GABA}_{\mathrm{A}}$ ergic synapses (Sassoe-Pognetto et al., 1995), it would mean that a majority of Neuroligin 3-GABA $\mathrm{A}_{\mathrm{A}} 2$ clusters are not equipped with Gephyrin.

Moreover, similar to Neuroligin 2, Neuroligin 3 associated equally with diverse $\mathrm{GABA}_{\mathrm{A}}$ receptor subsets containing the $\mathrm{GABA}_{\mathrm{A}} \alpha 1, \mathrm{GABA}_{\mathrm{A}} \alpha 2$ or $\mathrm{GABA}_{\mathrm{A}} \alpha 3$ subunits (Figure 3.14CF, quantified in 3.14E). Interestingly, Neuroligin 3 puncta were often found to decorate the 
soma of retinal ganglion cells, together with $\mathrm{GABA}_{\mathrm{A}}$ receptor clusters (Figure 3.14F, lower panel). In summary, the majority of Neuroligin 3 clusters at the IPL are localized at inhibitory postsynapses, and preferentially associate with $\mathrm{GABA}_{\mathrm{A}}$ - rather than glycine receptors.

A

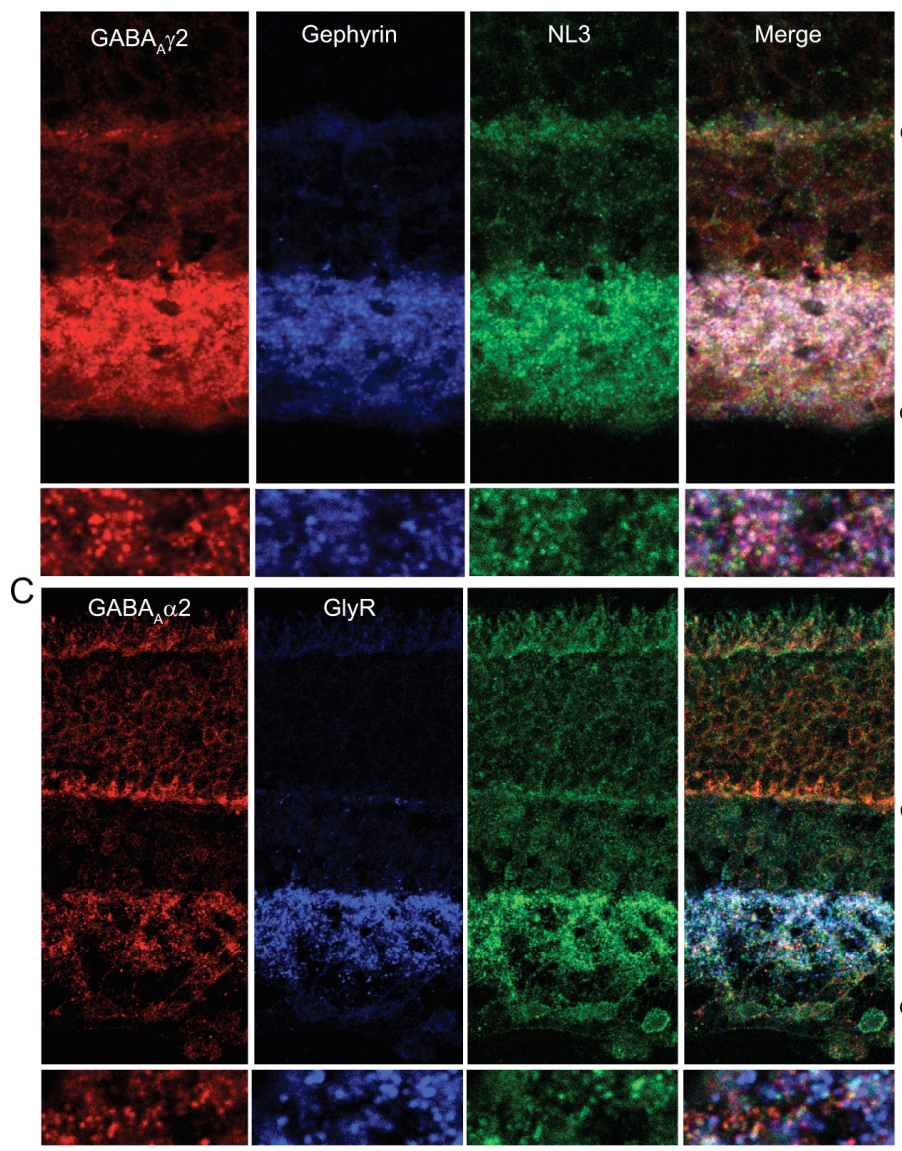

E

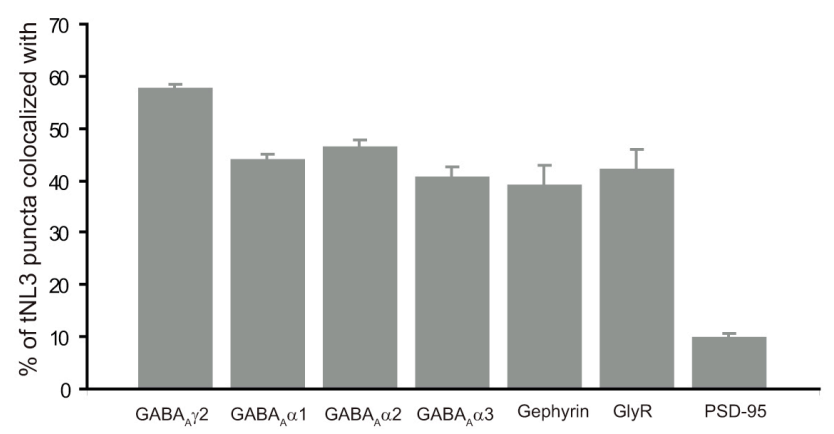

B

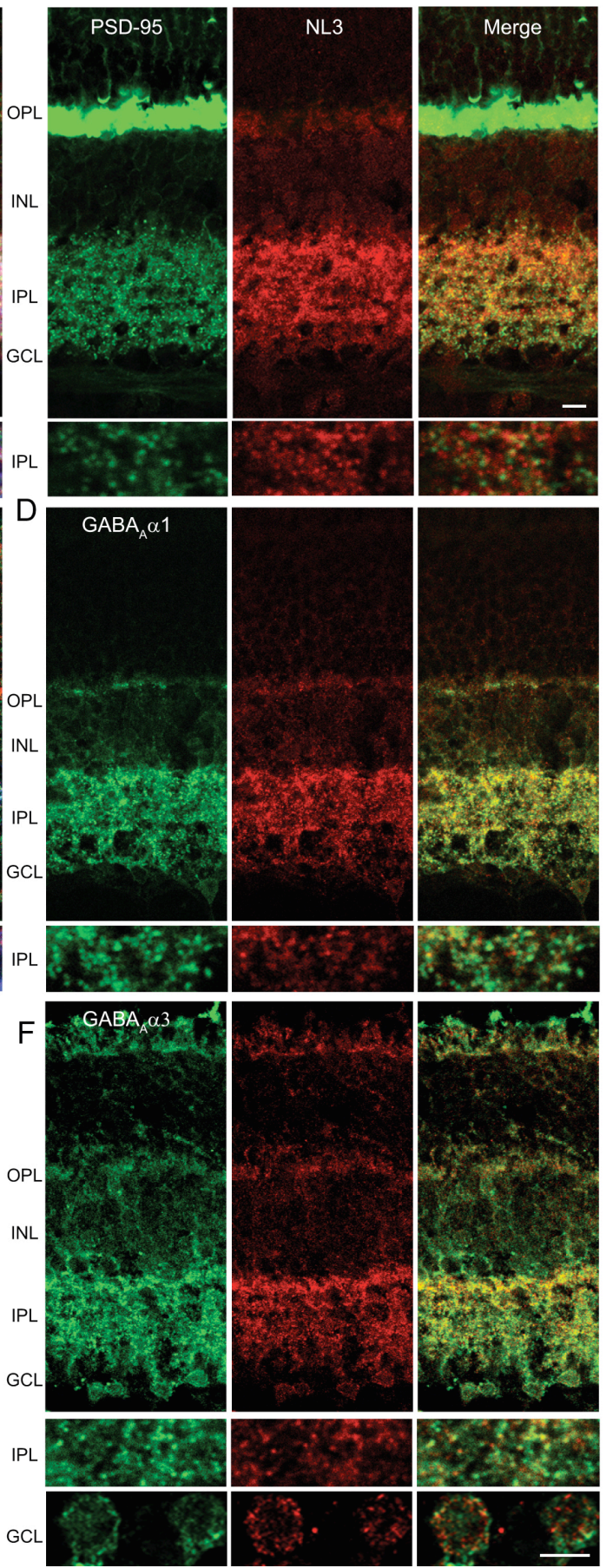

Figure 3.14: Localization of Neuroligin 3 in the mouse retina

To ascertain the distribution of Neuroligin 3 (NL3) at retinal synapses, immunolabelings were carried out for Neuroligin 3 together with excitatory $(\mathbf{B})$ and inhibitory $(\mathbf{A}, \mathbf{C}, \mathbf{D}, \mathbf{F})$ postsynaptic markers. Neuroligin 3 was devoid from a majority of excitatory postsynapses $(\mathbf{B}, \mathbf{E})$, but colocalized extensively with the ubiquitous $\mathrm{GABA}_{\mathrm{A}}$ receptor subunit: $\mathrm{GABA}_{\mathrm{A}} \gamma 2(\mathbf{A}, \mathbf{E})$. Further, Neuroligin 3 was also found at GlyR synapses $(\mathbf{C}, \mathbf{E})$ and associated with diverse $\mathrm{GABA}_{\mathrm{A}}$ receptor subtypes $\left(\mathrm{GABA}_{\mathrm{A}} \alpha 1, \alpha 2\right.$ and $\alpha 3)(\mathbf{C}-\mathbf{F}) . \mathrm{OPL}=$ outer plexiform layer; $\mathrm{INL}=$ inner nuclear layer; $\mathrm{IPL}=$ inner plexiform layer; $\mathrm{GCL}=$ ganglion cell layer. Scale bar $=5 \mu \mathrm{m}$. 


\subsubsection{Retinal architecture is preserved in the absence of Neuroligin 3}

To assess the integrity of the retina in the absence of Neuroligin 3, diverse cellular and synaptic markers were compared between wild-type (WT) and Neuroligin 3-deficient (NL3 KO) retinae (Figure 3.15). Rod bipolar and OFF-cone bipolar cell populations were assessed by labeling for PKC $\alpha$ (Haverkamp and Wassle, 2000) and Neurokinin 3 receptor (NK3-R) (Casini et al., 2000) respectively. Cholinergic and dopaminergic subsets of GABAergic amacrine cells were evaluated by labeling for vesicular acetylcholine transporter (VAChT) (Koulen, 1997) and tyrosine hydroxylase (TH) (Zhang et al., 2005) respectively. Ribbon synapses at the OPL, and conventional inhibitory synapses at the IPL were compared by labeling for Bassoon (Brandstatter et al., 1999; Dick et al., 2001). The entire GABAergic amacrine cell population was labeled with GAD 67 (Vardi and Auerbach, 1995; Haverkamp and Wassle, 2000) (Figure 3.15; GAD 65 showed the same result) and excitatory and inhibitory postsynapses were labeled with PSD-95 (Koulen et al., 1998b) and Gephyrin (Sassoe-Pognetto and Wassle, 1997) respectively. No alteration in the structural organization of the Neuroligin 3-deficient retina was observed (Figure 3.15).
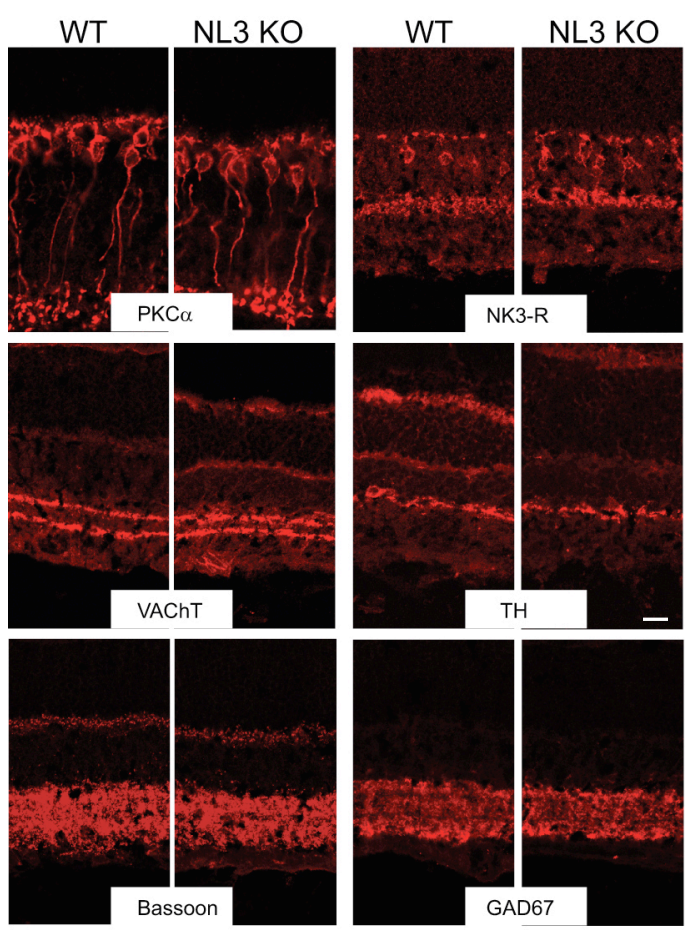

Figure 3.15: Architecture of the Neuroligin 3-deficient retina

Rod (PKC $\alpha$ ) and OFF-cone bipolar (NK3$\mathrm{R})$ cell populations, cholinergic (VAChT) and dopaminergic (TH) amacrine cells, GABAergic (GAD67) amacrine cells, ribbon synapses at the OPL and conventional synapses at IPL (Bassoon) and excitatory (PSD-95) and inhibitory (Gephyrin) postsynapses were compared between wild-type (WT) and Neuroligin 3deficient (NL3 KO) retinae. No alteration in the organization of the Neuroligin 3deficient retina was uncovered.

Scale bar $=10 \mu \mathrm{m}$.
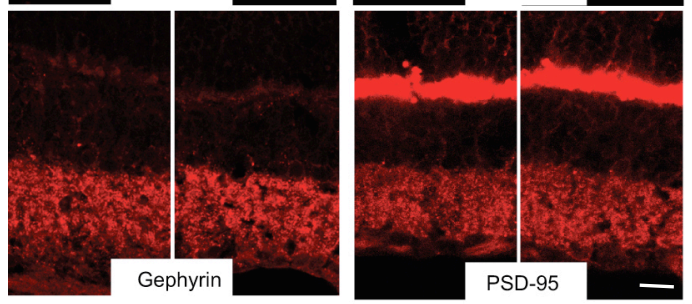


\subsubsection{Loss of Neuroligin 3 leads to a dramatic impairment in a $\mathrm{GABA}_{\mathrm{A}}$ receptor subset}

Since the majority of Neuroligin 3 puncta were localized at $\mathrm{GABA}_{\mathrm{A}}$ and GlyR postsynapses, the next step was to analyze these receptor populations in wild-type (WT) and Neuroligin 3deficient (NL3 KO) retinae. Diverse subsets of $\mathrm{GABA}_{\mathrm{A}}$ receptors, bearing the $\mathrm{GABA}_{\mathrm{A}} \alpha 1, \alpha 2$, $\alpha 3$ and/or $\gamma 2$ subunits were labeled for, in addition to the entire population of GlyRs (labeled by $\mathrm{mAb} 4 \mathrm{a}$ ) (Figure 3.16A), and their cluster density quantified at the IPL of WT and Neuroligin $3 \mathrm{KO}$ (Figure 3.16B). Interestingly, there was a specific reduction of $\mathrm{GABA}_{\mathrm{A}} \alpha 2$ subunit containing receptor clusters in the absence of Neuroligin $3\left(\mathrm{WT}_{\text {mean }}=131.07 \pm 8.56\right.$ puncta $/ 100 \mu \mathrm{m}^{2}$ of IPL; $\mathrm{KO}_{\text {mean }}=45.21 \pm 1.05$ puncta $/ 100 \mu \mathrm{m}^{2}$ of IPL; $\mathrm{n}=3$ pairs; $\mathrm{p}=0.01$ ) (Figure 3.16A, B), while the other $\mathrm{GABA}_{\mathrm{A}}$ receptor clusters remained comparable in both groups (Figure 3.16A,B). In addition, the total number of GlyR, Gephyrin and PSD-95 clusters also remained unperturbed in the Neuroligin 3-deficient retinae (Figure 3.16A, B). Hence, there is a specific impairment of $\mathrm{GABA}_{\mathrm{A}} \alpha 2$ receptor clusters in the Neuroligin 3-deficient retina.
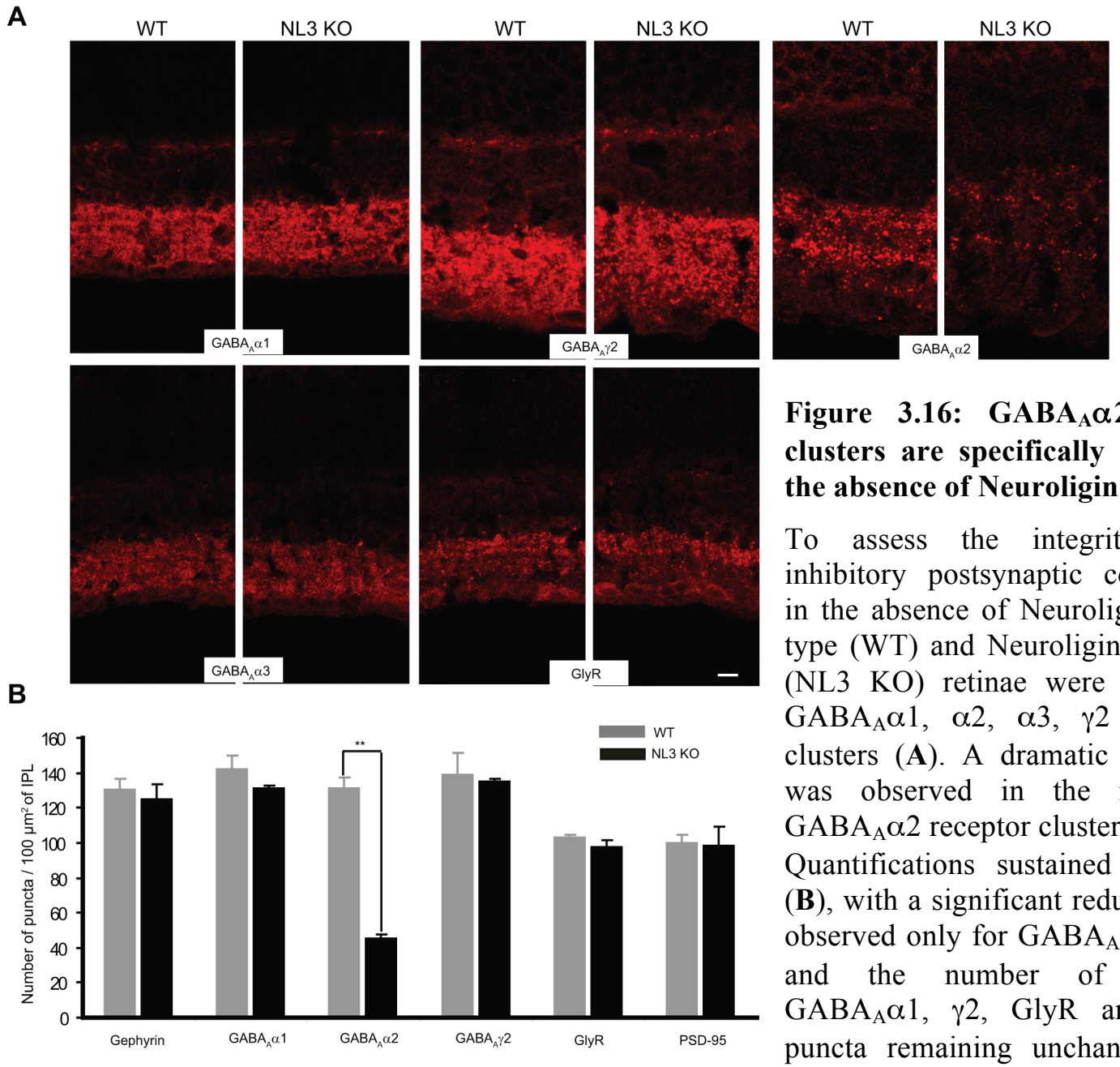

Figure 3.16: $\mathrm{GABA}_{\mathrm{A}} \alpha 2$ receptor clusters are specifically reduced in the absence of Neuroligin 3

To assess the integrity of the inhibitory postsynaptic compartment in the absence of Neuroligin 3, wildtype (WT) and Neuroligin 3-deficient (NL3 KO) retinae were labeled for $\mathrm{GABA}_{\mathrm{A}} \alpha 1, \alpha 2, \alpha 3, \gamma 2$ and GlyR clusters (A). A dramatic impairment was observed in the number of $\mathrm{GABA}_{\mathrm{A}} \alpha 2$ receptor clusters (A).

Quantifications sustained this result (B), with a significant reduction being observed only for $\mathrm{GABA}_{\mathrm{A}} \alpha 2$ clusters, and the number of Gephyrin, $\mathrm{GABA}_{\mathrm{A}} \alpha 1, \gamma 2$, GlyR and PSD-95 puncta remaining unchanged in the NL3 KO as compared to the WT. $(n=3$ pairs). Scale bar $=5 \mu \mathrm{m}$. 
To evaluate in further detail the glycinergic postsynaptic compartment, distinct subsets of GlyRs (containing GlyR $\alpha 1, \alpha 2, \alpha 3$ or $\alpha 4$ subunits) were labeled for in the WT and Neuroligin 3-deficient (NL3 KO) retinae (Figure 3.17). However, no alteration was observed in the Neuroligin $3 \mathrm{KO}$ samples, as compared to WT, for any of the GlyR subclasses.
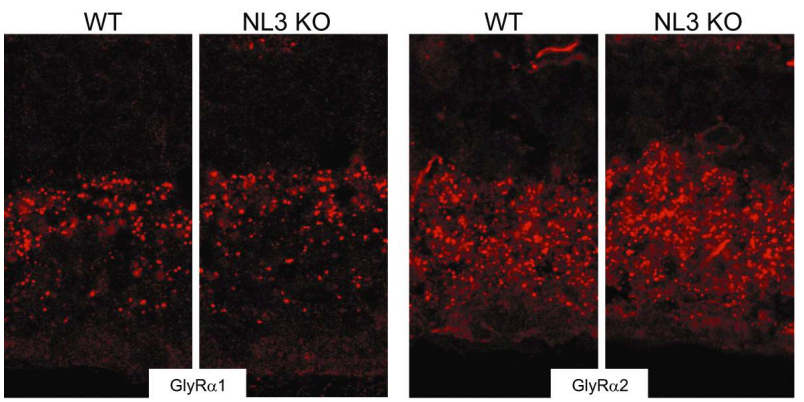

Figure 3.17: GlyR subsets in the Neuroligin 3-deficient retina

Distinct GlyR subclasses (GlyR $\alpha 1, \alpha 2, \alpha 3$ and $\alpha 4$ ) were labeled for in the wild-type (WT) and Neuroligin 3-deficient (NL3 KO) retinae. No alteration in the density or distribution of any of the GlyR subsets was observed for the NL3 KO.
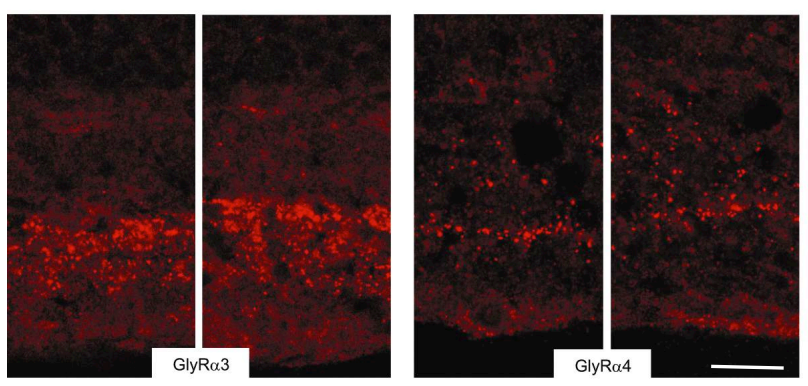

Taken together, the loss of Neuroligin 3 in the retina, did not lead to any alterations in the structural organization of the retina, neither with respect to the main (excitatory) signal transmission pathway nor the glycinergic modulatory circuit. However, it led to a specific reduction in the number of $\mathrm{GABA}_{\mathrm{A}}$ receptor clusters (those containing the $\alpha 2$-subunits). Whether this affects the integrity of visual processing in the Neuroligin 3-deficient animals, remains to be investigated. 


\subsection{Modulation of Neuroligin 4 expression in the retinae from Neuroligin 2-}

\section{deficient mice}

The characterization of Neuroligin 2,3 and 4 in the mouse retina, revealed that all these isoforms are preferentially associated with the inhibitory postsynaptic compartment of the IPL. Therefore, an important question to address was whether these Neuroligin isoforms could compensate for each other at the IPL. To this end, we analyzed the expression of Neuroligin 3 and 4 in the Neuroligin 2-deficient (NL2KO) retinae (Figure 3.18A). Surprisingly, in retina samples from Neuroligin 2-deficient animals, a significant upregulation of Neuroligin 4 was observed (Figure 3.18A, quantified in 3.18B). On the contrary the density of Neuroligin 3 puncta at the IPL of Neuroligin 2-deficient animals, remained unchanged (Figure 3.18A, quantified in 3.18B). Not only were Neuroligin 4-positive clusters 2-3 times more abundant $\left(\mathrm{WT}_{\text {mean }}=26.00 \pm 4.80 \mathrm{NL} 4\right.$ puncta $/ 100 \mu \mathrm{m}^{2}$ of IPL; NL2 $\mathrm{KO}_{\text {mean }}=72.51 \pm 5.14 \mathrm{NL} 4$ puncta/ $100 \mu \mathrm{m}^{2}$ of IPL; $\mathrm{n}=7$ pairs; $\mathrm{p}<0.0001$ ) in the Neuroligin $2 \mathrm{KO}$ retinae as compared to the wild-type littermates (WT) (Figure 3.18B), but also their distribution was more widespread at the IPL of the Neuroligin 2 KO (Figure 3.18A). Conversely, the expression levels of Neuroligin 2 remained unaltered in the Neuroligin 4-deficient (NL4 KO) retinae (Figure 3.18C, quantified in 3.18D).

The synaptic distribution of the upregulated Neuroligin 4 puncta at the IPL of Neuroligin 2 KO retinae was next analyzed. In the Neuroligin 2-deficient retinae, the upregulated Neuroligin 4 puncta remained independent from PSD-95 labeled clusters and continued to display a preferential association with GlyRs $(73 \pm 3 \%$ of upregulated Neuroligin 4 puncta still associated with GlyR clusters) rather than $\gamma 2$-bearing $\mathrm{GABA}_{\mathrm{A}}$ receptors (only $18 \pm 4 \%$ of upregulated Neuroligin 4 puncta colocalized with $\mathrm{GABA}_{\mathrm{A}} \gamma 2$ clusters). Of note, the absolute number of $\gamma 2$ puncta that associated with Neuroligin 4 increased significantly from $0.71 \pm$ $0.2 \%$ in the WT to $3.18 \pm 0.4 \%$ in the Neuroligin $2 \mathrm{KO}$ ( $\mathrm{p}=0.0006$ ), as did the proportion of GlyR synapses that colocalized with Neuroligin 4, which increased to $44 \pm 6 \%$ in Neuroligin 2 KO $(p=0.0106)$. This indicates that the specificity of Neuroligin 4 association with glycinergic postsynapses is unaltered in Neuroligin 2 KO.

As glycinergic amacrine cells are also upregulated in the Neuroligin 2-deficient retinae (Figure 3.5D), the dramatic increase in the density of Neuroligin 4 puncta at the IPL of the Neuroligin $2 \mathrm{KO}$ might reflect an alteration of the glycinergic circuitry, rather than a compensation for the absence of Neuroligin 2. 
A

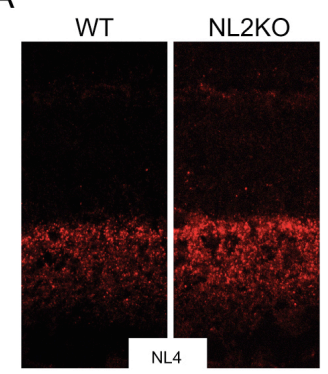

C

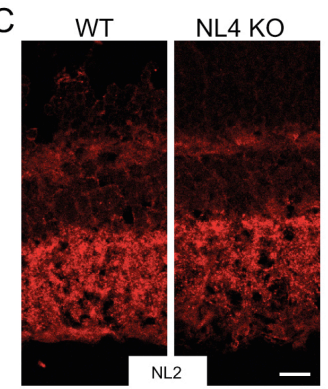

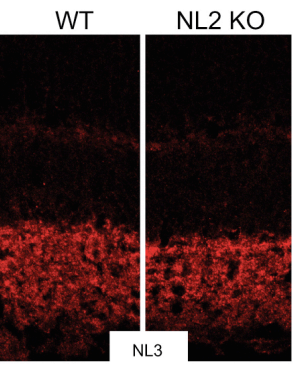
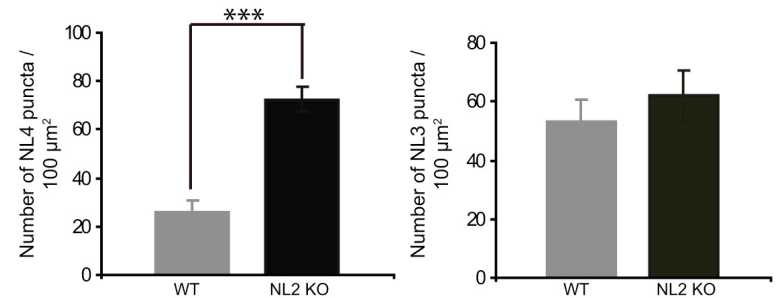

D

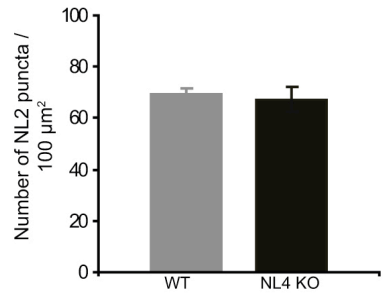

Figure 3.18: Upregulation of Neuroligin 4 in the Neuroligin 2 deficient retina

Expression levels of Neuroligin 4 (NL4) and Neuroligin 3 (NL3) were evaluated in wild-type (WT) and Neuroligin 2-deficient (NL2KO) retinae (A). A significant upregulation of Neuroligin 4 puncta number was observed at the IPL of Neuroligin 2-deficient retinae (B), while Neuroligin 3 levels remained unaltered $(\mathbf{A}, \mathbf{B})$.

In the Neuroligin 4-deficient retinae (NL4 KO) however, the levels of Neuroligin 2 (NL2) remained unaltered $(\mathbf{C}, \mathbf{D})$.

Scale bar $=10 \mu \mathrm{m}$. 


\section{Discussion}

In this study, the distribution and function of three different Neuroligin isoforms (Neuroligin 2-4) has been investigated in the mouse retina. Interestingly, none of the Neuroligin isoforms studied in this project were enriched at the excitatory postsynaptic compartment of the retina; rather, they were found to occupy distinct subsets of inhibitory (GABA/glycinergic) postsynapses.

Neuroligin 2 was characterized at GABAergic postsynapses of the retina, and a loss of Neuroligin 2 was paralleled by a dramatic impairment in specific $\mathrm{GABA}_{\mathrm{A}}$ receptor subsets, together with a subtle alteration in the glycinergic inhibitory circuit. Consequently, visual processing was affected, and retinal ganglion cells from Neuroligin 2-deficient animals apportioned a larger fraction of their response spikes for baseline (non-stimulus related) activity. Taken together, loss of Neuroligin 2 impairs the inhibitory surround at the IPL, which is detrimental to the stimulus-coding efficacy of the ganglion cells.

Similar to Neuroligin 2, a majority of Neuroligin 3 is associated with GABA $_{\mathrm{A}}$ receptor containing synapses, although $\sim 40 \%$ of Neuroligin 3 clusters were also localized at GlyRbearing synapses of the IPL. Consequently, a loss of Neuroligin 3 was found to be more detrimental to the integrity of the GABAergic circuit, with a specific impairment in a subset of $\mathrm{GABA}_{\mathrm{A}}$ receptors at the IPL of Neuroligin 3-deficient animals. Interestingly, this subset is distinct from the $\mathrm{GABA}_{\mathrm{A}}$ receptor classes affected by a loss of Neuroligin 2: $\mathrm{GABA}_{\mathrm{A}} \alpha 2$ clusters are reduced in Neuroligin 3-deficient animals, when $\mathrm{GABA}_{\mathrm{A}} \gamma 2$ and $\alpha 3$ clusters are affected by a deficiency of Neuroligin 2, indicating that the two Neuroligin isoforms are regulating distinct GABAergic postsynapses in the retina.

In contrast to Neuroligin 2 and 3, Neuroligin 4 is distributed specifically at the glycinergic postsynaptic compartment of the retina. However, the expression of Neuroligin 4 is not as robust as Neuroligin 2 or 3 , and Neuroligin 4 is located at only a fraction $(\sim 21 \%)$ of GlyR synapses. Accordingly, a loss of Neuroligin 4 causes a subtle impairment in a subset of glycinergic postsynapses containing GlyR subunits with fast kinetic properties. Functional analyses of inhibitory, glycinergic currents recorded from ganglion cells revealed a slower decay time course in the absence of Neuroligin 4. However, when ganglion cell responses to light flashes were analyzed, no observable alteration was detected in baseline (non-stimulus related) or light-driven spiking activity. Thus, Neuroligin 4 equips a subset of glycinergic postsynapses in the retina, and a loss of Neuroligin 4 is detrimental to the functioning of a small proportion of GlyR synapses. 
In summary, Neuroligins 2 and 3 control GABA receptor clustering at the inner synaptic layer of the retina and thus govern the inhibitory surround that shapes the activity of the retinal ganglion cells. Neuroligin 4, on the other hand, plays a small yet significant role in tuning glycinergic inhibition at the IPL, which may have limited consequences on information processing as glycinergic amacrine cells are small-field cells that principally control local inhibitory interactions at the IPL, rather than ganglion cell receptive fields. 


\subsection{Distribution of Neuroligin isoforms in the retina}

There are four Neuroligin isoforms expressed in rodents (Ichtchenko et al., 1995; Ichtchenko et al., 1996; Jamain et al., 2008). Before the start of this study, Western blot analyses of retina homogenates using isoform-specific antibodies established the presence of all four isoforms in the mouse retina (F. Varoqueaux, unpublished observations). The expression and distribution of the different Neuroligin isoforms in the retina were studied by immunohistochemistry. While, a convincing labeling for Neuroligin 1 was difficult to obtain with the fixation and immunolabeling protocols that were used, a robust labeling pattern was obtained for Neuroligins 2, 3 and 4 using the respective isoform-specific antibodies. The validity of these labelings was established by the absence of a specific signal in the corresponding deletionmutant mice.

In the retina, Neuroligin 2 was localized preferentially at GABAergic, rather than glycinergic inhibitory postsynapses. Previous studies have demonstrated the localization of Neuroligin 2 exclusively at inhibitory postsynapses (Varoqueaux et al., 2004). In keeping with its distribution in the brain, Neuroligin 2 did not associate with excitatory postsynaptic clusters (Figure 3.2B, E) and localized specifically to inhibitory GABAergic postsynapses in the retina, as demonstrated by robust colocalization with the ubiquitous $\mathrm{GABA}_{\mathrm{A}}$ receptor subunit $\mathrm{GABA}_{\mathrm{A}} \gamma 2$ at both the outer and inner synaptic layers (OPL and IPL) (Figure 3.2A, E). Interestingly, Neuroligin 2 was not ubiquitously distributed at all inhibitory postsynapses of the retina, and only a limited colocalization of Neuroligin 2 was observed with GlyRs, indicating that a majority of glycinergic postsynapses in the retina are devoid of Neuroligin 2. A recent study examined the expression pattern of Neuroligin 2 in the rat retina by in situ hybridization, and found the highest levels of expression at the ganglion cell layer, indicating a robust localization of Neuroligin 2 at ganglion cell dendrites (Lui et al., 2010). Further, the authors report close appositions between Neuroligin 2 puncta and VGAT at the IPL, corroborating the results from our study that Neuroligin 2 is localized at inhibitory postsynapses of the retina.

Comparable to Neuroligin 2, Neuroligin 3 was localized at inhibitory postsynapses of the retina. This was the first in vivo characterization of Neuroligin 3 distribution in the CNS. Previous in vitro studies have indicated a prominent association of Neuroligin 3 with excitatory postsynapses (Graf et al., 2004). However, clustering of Neuroligin 3 in hippocampal neurons by Neurexin-expressing COS cells results in the co-aggregation of Neuroligin 3 with both glutamatergic and GABAergic scaffolding proteins (Budreck and 
Scheiffele, 2007), and in hippocampal cultures, Neuroligin 3 puncta were found to colocalize with both excitatory and inhibitory synaptic markers (Budreck and Scheiffele, 2007). In the retina, however, the majority of Neuroligin 3 puncta were absent from excitatory postsynaptic clusters ( $\sim \%$ of Neuroligin 3 puncta were also immunopositive for PSD-95 at the IPL). Rather Neuroligin 3 puncta associated robustly with different subsets of inhibitory postsynapses ( $\sim 55 \%$ with $\mathrm{GABA}_{\mathrm{A}} \gamma 2$ and $\sim 40 \%$ with GlyR subunits) (Figure 3.14$)$. This indicates that in the retina Neuroligin 3 may regulate inhibitory processing at the IPL, and may not have a role in the direct excitatory (ribbon-synapses dominated) pathway.

An important open question is if Neuroligin 2 and 3 are present at the same GABAergic postsynapses of the IPL. Indeed in hippocampal cultures about $60 \%$ of Neuroligin 2-Gephyrin clusters also contain Neuroligin 3 (Budreck and Scheiffele, 2007), and, coimmunoprecipitation studies revealed the presence of Neuroligin 2-3 complexes in brain extracts (Budreck and Scheiffele, 2007). So far, however, technical difficulties (as all the available Neuroligin antibodies have been raised in the same species) have precluded the analysis of Neuroligin 2-3 co-distribution in the retina. The direct conjugation of one of the Neuroligin antibody to a fluorophore may help to address this point in the future.

Recent collaborative studies analyzing the distribution of Neuroligin 3 in the brain have revealed a preferential association of Neuroligin 3 with Gephyrin at the granule cell layer of the cerebellum, at the internal plexiform layer of the olfactory bulb and in the brainstem (M. Sassoe-Pognetto and A. Patrizi, unpublished observations). This indicates that Neuroligin 3 may be predominantly associated with inhibitory postsynapses in the brain as well, and the region-specific localization of Neuroligin 3 is currently being investigated.

The in vivo distribution of Neuroligin 3, in the retina as well as in the brain, thus does not support the data from earlier in vitro assays, and indicate that Neuroligin 3 could have a role specifically at inhibitory postsynapses of the CNS.

Although several mutations in Neuroligin 4 have been linked to cases of autism and mental retardation (Jamain et al., 2003; Laumonnier et al., 2004; Jamain et al., 2008; Zhang et al., 2009), the distribution and function of Neuroligin 4 at CNS synapses had not been studied when the present work had started. In vitro, clustering of overexpressed Neuroligin 4 by antibodies leads to the co-clustering of the excitatory postsynaptic protein PSD-95 but not of the inhibitory scaffold protein Gephyrin (Graf et al., 2004), indicating that Neuroligin 4 may be enriched at excitatory postsynapses of the CNS.

In the retina, however, Neuroligin 4 colocalizes specifically with postsynaptic GlyR clusters and no overlap is seen with the excitatory postsynaptic marker PSD-95 (Figure 3.9). It is 
interesting to note that none of the Neuroligin isoforms analyzed in this study localized at excitatory postsynapses of the retina, the majority of which are ribbon synapses. As ribbon synapses bear unconventional presynaptic proteins (discussed in 1.2.2), it might be that Neuroligins are not needed for the formation/function of these specialized synapses. However, further experiments analyzing the distribution of Neuroligin 1 will be necessary to answer this question.

In the retina, Neuroligin 4 was the first isoform to display selectivity towards glycinergic synapses, with a vast majority of Neuroligin 4 colocalizing with GlyR clusters (Figure 3.9). In contrast, only a limited number of GlyR clusters were immunopositive for Neuroligin 4 at the IPL, indicating that Neuroligin 4 equips only a subset $(\sim 21 \%)$ of all glycinergic synapses in the retina. However, Neuroligin 2 and 3 were also found to localize at subsets of GlyRcontaining synapses, and it remains to be seen whether the different Neuroligin isoforms occupy distinct subpopulations of glycinergic synapses, and whether all glycinergic synapses bear Neuroligins. To address this question, it would be essential to perform triple labeling for GlyRs together with different combinations of Neuroligin isoforms (Neuroligin 2/3; 2/4 and 3/4), but technical difficulties have precluded this analysis so far.

Interestingly, recent immunohistochemical studies in the department have examined the distribution of Neuroligin 4 throughout the CNS, and have found Neuroligin 4 to be enriched in the spinal cord and brainstem. In both regions, Neuroligin 4 puncta colocalized extensively with the inhibitory scaffold protein Gephyrin and with GlyR subunits (M. Hammer and F. Varoqueaux, unpublished observations). Thus the preferential association of Neuroligin 4 with GlyRs is not restricted to the retina but present in other regions of the CNS as well. These in vivo distribution data (in the retina as well as in the brainstem and spinal cord) thus, do not support the in vitro overexpression assays and underscore the involvement of Neuroligin 4 at glycinergic postsynapses of the CNS.

In support of a possible role of Neuroligin 4 at the inhibitory postsynapse, yeast two hybrid assays have shown that Neuroligin 4 can bind to the inhibitory postsynaptic protein Gephyrin (Poulopoulos et al., 2009), and can, in addition, interact with Collybistin (T. Soykan and F. Varoqueaux, unpublished observations), a protein implicated in the recruitment of Gephyrin to postsynaptic clusters (Kins et al., 2000). Aspects of the mechanisms underlying the interaction of different Neuroligin isoforms with inhibitory postsynaptic proteins were recently elucidated for Neuroligin 2 (Poulopoulos et al., 2009), (see 4.2.1), and Neuroligin 4 may operate in a similar manner. The molecular mechanisms underlying the specificity of Neuroligin isoforms for GABA- vs. glycinergic synapses is currently being investigated. 


\subsection{Postsynaptic architecture in the Neuroligin-deficient retina}

The absence of different Neuroligin isoforms, differentially affected the composition of the inhibitory postsynaptic compartment: A loss of Neuroligin 2 had a predominant effect on the clustering of $\mathrm{GABA}_{\mathrm{A}}$ receptors containing the $\gamma 2$ and $\alpha 3$ subunits, while the absence of Neuroligin 3 led to a specific loss of $\alpha 2$ subunit-containing $G_{A B A}$ receptor subsets. This implies that, like Neuroligin 2, Neuroligin 3 is crucial for the stability of GABAergic postsynapses, albeit for a different subpopulation of $\mathrm{GABA}_{\mathrm{A}}$ receptors. Quite distinct from the other two isoforms, a deficiency of Neuroligin 4 was paralleled with a subtle deficit in the glycinergic postsynaptic compartment in a specific subset of GlyR subunits that confer fast kinetics to the receptor.

\subsubsection{GABA receptors}

The specificity of Neuroligin 2 for $\mathrm{GABA}_{\mathrm{A}}$ - but not glycine receptor-bearing synapses in the retina, indicates a preferential link between Neuroligin 2 and $\mathrm{GABA}_{\mathrm{A}}$ receptors. Further, at the IPL two-thirds of $\mathrm{GABA}_{\mathrm{A}}$ receptors associate with Neuroligin 2 at the inhibitory postsynaptic compartment, implying that Neuroligin 2 could modulate the functioning of a majority of $\mathrm{GABA}_{\mathrm{A}}$ receptors. The absence of Neuroligin 2 resulted in impaired clustering of $\gamma 2-$ containing $\mathrm{GABA}_{\mathrm{A}}$ receptors at the IPL (Figure 3.6). The density of $\mathrm{GABA}_{\mathrm{A}} \gamma 2$ clusters at the IPL was reduced in the absence of Neuroligin 2 and the clusters that remained were dimmer (Figure 3.6). $\mathrm{GABA}_{\mathrm{A}} \gamma 2$ is essential for the functional integrity of $\mathrm{GABA}_{\mathrm{A}}$ receptors (Schweizer et al., 2003). Hence, a loss of $\mathrm{GABA}_{\mathrm{A}} \gamma 2$ clusters in the Neuroligin 2-deficient retina indicates that there are fewer functional $\mathrm{GABA}_{\mathrm{A}}$ receptors in the absence of Neuroligin 2. While the GABA $\mathrm{A} 2$ subunit is not required for the assembly of GABA-gated ion channels (Gunther et al., 1995), the presence of the $\gamma$ - subunit $(\gamma 2$ or $\gamma 3)$ is vital for postsynaptic clustering of the receptor (Essrich et al., 1998; Baer et al., 1999). Furthermore, the $\gamma 2$ subunit seems to be essential for the functioning of $\mathrm{GABA}_{\mathrm{A}}$ receptors, since single-channel conductance, open probability and duration are markedly reduced in the absence of the $\gamma 2$ subunit (Lorez et al., 2000). In addition, postsynaptic $\mathrm{GABA}_{\mathrm{A}}$ receptors depend on the $\gamma 2$ subunit for long-term maintenance, as inactivation of the $\gamma 2$ locus leads to a rapid decline in $\mathrm{GABA}_{\mathrm{A}}$ receptors (Schweizer et al., 2003), underscoring the potential role of $\gamma 2$ in normal recycling of $\mathrm{GABA}_{\mathrm{A}}$ receptors and hence plasticity.

In addition, a loss of Neuroligin 2 differentially affected the clustering of the other $\mathrm{GABA}_{\mathrm{A}}$ receptor subunits as well: as $\alpha 1$ clusters were dimmer, $\alpha 2$ clusters remained unaltered, and $\alpha 3$ 
clusters were reduced in numbers (Figure 3.6). $\mathrm{GABA}_{\mathrm{A}} \alpha 1$ and $\alpha 3$ subunits associate extensively with $\gamma 2$ to form $\mathrm{GABA}_{\mathrm{A}}$ receptors in the retina, while $\mathrm{GABA}_{\mathrm{A}} \alpha 2$ subunits associate prominently with the $\delta$ subunit to form $\mathrm{GABA}_{\mathrm{A}}$ receptors in the retina (discussed in 1.2.3). Altogether, the alterations in $\mathrm{GABA}_{A} \alpha 1, \alpha 3$ and $\gamma 2$ receptor subunits, point towards a deficit in the synaptic clustering of $\mathrm{GABA}_{\mathrm{A}}$ receptors and indicate that Neuroligin 2 is crucial for maintaining the integrity of $\mathrm{GABA}_{\mathrm{A}}$ receptor clusters at the IPL.

Recent yeast two-hybrid experiments have shown that Neuroligin 2 interacts with the inhibitory scaffolding protein Gephyrin via a conserved cytoplasmic motif (Poulopoulos et al., 2009). However, this motif is present in all Neuroligin isoforms, and thus cannot solely account for the specificity of Neuroligin 2 to inhibitory postsynapses. Interestingly, Neuroligin 2 (but not Neuroligin 1) also interacts with and activates Collybistin, thereby targeting Gephyrin-Collybistin complexes to the plasma membrane (Poulopoulos et al., 2009). This latter interaction with Collybistin is a key factor in the functional specificity of Neuroligin 2 for inhibitory postsynapses. In keeping with an association of Neuroligin 2 with Gephyrin, in the CA1 region of the hippocampus, the absence of Neuroligin 2 does not affect the number of inhibitory synaptic contacts but causes a lamina-specific reduction (selectively at the Stratum pyramidale) in Gephyrin and $\mathrm{GABA}_{\mathrm{A}} \gamma 2$ receptor clusters (Figure 3.8) (Poulopoulos et al., 2009). Similar findings were made in the dentate gyrus (Jedlika et al), substantiating the notion that in the hippocampus, Neuroligin 2 controls the clustering of $\mathrm{GABA}_{\mathrm{A}}$ receptor subsets by gephyrin-dependent mechanisms. This notion is consistent with the findings of Blundell et al., (2009), who show that the numbers of symmetric synapses in the CA1 region of WT and Neuroligin $2 \mathrm{KO}$ mice, as estimated by electron microscopy, are identical.

It is noteworthy, that in the hippocampus, a loss of Neuroligin 2 is detrimental to the integrity of a subset of $\mathrm{GABA}_{\mathrm{A}}$ receptor clusters, the perisomatic $\mathrm{GABA}_{\mathrm{A}}$ clusters, and this deficit is paralleled with an equivalent reduction of Gephyrin clusters as well. Strikingly, when wildtype and Neuroligin 2-deficient hippocampal cultures were labeled for Gephyrin and the ubiquitous presynaptic marker Synapsin, a specific loss of Gephyrin clusters was observed also at perisomatic sites of Neuroligin 2-deficient neurons, while the Gephyrin clustering at the dendritic compartment remained unaffected (Poulopoulos et al., 2009). Furthermore, functional analysis of the GABAergic mIPSCs from the hippocampus of Neuroligin 2deficient animals, displayed events with slower onset, indicating a selective loss of the fastonset perisomatic events (Poulopoulos et al., 2009). These results indicate that at the 
hippocampus, Neuroligin 2 controls the assembly of perisomatic inhibitory GABAergic postsynapses through Gephyrin.

In the retina, however, the partial association of Neuroligin 2 with Gephyrin (Figure 3.2C, E) indicates that Neuroligin 2 also acts independently of Gephyrin at the GABAergic postsynapses of the IPL. Consistently, absence of Gephyrin in the retina disrupts the clustering of all GlyRs, but of only half of the GABA ${ }_{A} 2$ receptors (Fischer et al., 2000). Moreover, recent ultrastructural analyses of brainstem sections from Gephyrin null mutant mice have shown that Gephyrin is not required for the morphological differentiation of inhibitory postsynapses and that even in the absence of Gephyrin, the postsynaptic specializations of inhibitory synapses are maintained. Furthermore, in hippocampal cultures from these Gephyrin KO mice, Neuroligin 2 puncta are still appositioned to GAD65-labeled inhibitory presynapses (O'Sullivan et al., 2009). Additionally, in cultured cerebellar granule cells Neuroligin 2 accelerates inhibitory postsynapse maturation by promoting the incorporation of the $\alpha 1$-subunit containing $\mathrm{GABA}_{\mathrm{A}}$ receptors (Fu and Vicini, 2009) and Neuroligin 2 distribution is intact at inhibitory synapses in the $\mathrm{GABA}_{\mathrm{A}} \alpha 1 \mathrm{KO}$ mouse (Patrizi et al., 2008). These results place Neuroligin 2 upstream of GABA receptor and Gephyrin in the mechanisms underlying inhibitory synapse formation and maintenance, and indicate that Neuroligin 2 could interact directly with $\mathrm{GABA}_{\mathrm{A}}$ receptors to promote their proper clustering at the postsynapse. In support of this mechanism, recent in vitro assays have indicated an interaction between Neuroligin 2 and $\mathrm{GABA}_{\mathrm{A}}$ receptors (Dong et al., 2007) and the tangential distribution of Neuroligin 2 at inhibitory synapses observed here at the ultrastructural level (Figure 3.4) is compatible with such a scenario. Taken together, the observations from the Neuroligin 2-deficient retina indicate that Neuroligin 2 is crucial for the integrity of $\mathrm{GABA}_{\mathrm{A}}$ receptors, and could work independently of Gephyrin as a specific organizer of the GABA receptor complement.

Residual $\gamma 2$ clusters observed upon Neuroligin 2 deletion likely correspond to the fraction of $\mathrm{GABA}_{\mathrm{A}}$ receptor clusters that do not associate with Neuroligin 2. They could also represent non-synaptic clusters, or associate with other Neuroligin isoforms such as Neuroligin 3. It is worth mentioning, that Neuroligin 3 levels are not upregulated in the Neuroligin 2-deficient retina. This indicates that Neuroligin 3 does not compensate for the loss of Neuroligin 2 at $\gamma 2$ containing $\mathrm{GABA}_{\mathrm{A}}$ synapses, even though it equips a large proportion of $\mathrm{GABA}_{\mathrm{A}} \gamma 2$ synapses at the IPL (Figure 3.14). In this regard, it is likely that Neuroligin 2 and 3 are localized at the same GABAergic postsynapses, where they may be involved in different functions, with 
Neuroligin 2 being solely responsible for maintaining the structure/functionality of $\mathrm{GABA}_{\mathrm{A}}$ receptors (via the $\gamma 2$ subunit). What speaks against the localization of Neuroligin 2 and 3 at the same $\mathrm{GABA}_{\mathrm{A}}$ postsynapse is the observation that a loss of Neuroligin 3 is accompanied with a deficit in a distinct $\mathrm{GABA}_{\mathrm{A}}$ receptor subset, those that contain the $\alpha 2$-subunit (Figure 3.16). As $\mathrm{GABA}_{\mathrm{A}} \alpha 2$ subunits have been shown to associate with $\delta$ or $\gamma 2$ subunits in the retina (Wassle et al., 1998), and as there was no change in the density of $\gamma 2$ clusters in the absence of Neuroligin 3, it will be important in future to assess the integrity of the $\mathrm{GABA}_{\mathrm{A}} \delta$ subunits upon Neuroligin 3 deletion. Taken together, the present results indicate that both these Neuroligin isoforms could occupy distinct $\mathrm{GABA}_{\mathrm{A}}$ receptor subsets, and thus might have divergent contributions to the maintenance of the inhibitory surround at the IPL.

\subsubsection{Glycine receptors}

The absence of Neuroligin 4 from the retina did not lead to any dramatic morphological alterations. In particular the main excitatory transmission pathway and the GABAergic circuit remained unperturbed in the Neuroligin 4-deficient retinae (Figure 3.10). However, at the IPL of the Neuroligin $4 \mathrm{KO}$, there was a small but consistent reduction in the density of GlyR clusters containing the $\alpha 1$ subunit (16.7\% loss in the KO as compared to the WT) (Figure 3.11). As all GlyRs in the retina depend on Gephyrin for postsynaptic clustering (Fischer et al., 2000), one important question to address in the future would be whether the loss of GlyR $\alpha 1$ in the Neuroligin 4-deficient retina is paralleled by an equivalent reduction in the density of Gephyrin clusters. This would provide insight into the molecular mechanism underlying Neuroligin 4 function at glycinergic postsynapses. However, it might be difficult to evaluate a subtle deficit in the density of Gephyrin clusters, as Neuroligin 4 is localized at a subpopulation of GlyR postsynapses ( 21\%), whereas Gephyrin is ubiquitously localized at all glycinergic postsynapses of the IPL.

In contrast, the absence of Neuroligin 2 and 3 did not lead to any visible alteration in the integrity of GlyR postsynapses (Figure 3.6 and Figure 3.17 respectively) although they associate with GlyRs to some extent. As all Neuroligins interact with Gephyrin (Poulopoulos et al., 2009), and as Gephyrin mediates the clustering of all GlyRs (Fischer et al., 2000), it will be important in the future to assess why only the loss of Neuroligin 4 affects the density of GlyRs at the IPL. 


\subsection{Retinal architecture in the absence of Neuroligin}

A loss of Neuroligin 3 or 4 did not lead to any alterations in the retinal cell populations or their lamination profile (Figure 3.15 and Figure 3.10 respectively). In contrast, a loss of Neuroligin 2 led to an increased expression of GlyT1 (Figure 3.5D, 3.6D). GlyT1 is a membrane associated $\mathrm{Na}^{+}-\mathrm{Cl}^{-}$glycine uptake transporter (Zafra et al., 1995), expressed on glycinergic amacrine cells, where it mediates the (fast) uptake of glycine from the synaptic cleft (Pourcho and Goebel, 1985; Wassle et al., 1986).

In the retina, glycine and GABA inhibitory networks develop in parallel (Kerschensteiner and Wong, 2008) and have been attributed specific roles. Glycinergic transmission mediates vertical inhibition (Wassle, 2004) and shapes the temporal properties of ganglion cells (O'Brien et al., 2003) while GABAergic transmission mediates lateral surround inhibition (Wassle, 2004; Majumdar et al., 2007) and shapes the spatial properties of ganglion cells (Dong and Werblin, 1998). The observed alteration in GlyT1 expression might reflect some adaptive remodeling of the retinal network, resulting indirectly from the Neuroligin 2 loss and / or the GABAergic network deficit. Further experiments will be required to determine whether these alterations correspond to a true increase in the dendritic coverage by glycinergic amacrine cells, or reflect an increased level of GlyT1 expression. Further, it will be important to evaluate whether the alteration in GlyT1 expression could also contribute to the functional deficits observed in the Neuroligin $2 \mathrm{KO}$ (discussed in 4.4.1). Monitoring the development of the GABA- and glycinergic circuits in the absence of Neuroligin 2 may help to understand the origin and role of this GlyT1 alteration.

Interestingly, Neuroligin 4 was strongly upregulated in the Neuroligin 2-deficient retina (Figure 3.18). The three Neuroligin isoforms (Neuroligin 2-4) occupy distinct subsets of inhibitory postsynapses - Neuroligin 2 localized at $\mathrm{GABA}_{\mathrm{A}}$ rather than GlyR postsynapses, Neuroligin 3 distributed in a 3:2 ratio between GABA $_{\mathrm{A}}$ and GlyR postsynapses and Neuroligin 4 overwhelmingly localized at glycinergic postsynapses. It is thus surprising that a loss of Neuroligin 2 in the retina is accompanied by a strong upregulation of Neuroligin 4 and not Neuroligin 3. Although a compensation by Neuroligin isoforms has so far not been reported, all Neuroligins have the same structural (binding) properties, and so could potentially be able to replace one another. The Neuroligin 4 upregulation observed by immunohistochemistry was further validated by Western blot analysis using retina homogenates from wild-type and Neuroligin 2-deficient animals. Upon quantification, Neuroligin 4 levels were significantly increased in retina homogenates from Neuroligin 2 (NL2) KO animals (expressed as 
Neuroligin 4/actin ratio; WT: $3 \pm 1.9, \mathrm{n}=5$; NL2 KO: $6.33 \pm 1.7, \mathrm{n}=5 ; \mathrm{p}=0.0279$ ) (experiments performed by Frederique Varoqueaux and Mrinalini Hoon).

One could hypothesize that these upregulated Neuroligin 4 clusters may associate with $\mathrm{GABA}_{\mathrm{A}}$ receptors, if Neuroligin 4 is replacing Neuroligin 2 at the synapse. Yet, Neuroligin 4 puncta in the Neuroligin 2-deficient retina still retain their preferential affiliation to glycinergic postsynapses, and do not "mislocalize" to $\mathrm{GABA}_{\mathrm{A}}$ receptor-containing postsynapses, that are dramatically impaired in the absence of Neuroligin 2 . This indicates that Neuroligin 4 does not directly compensate for the absence of Neuroligin 2 at the synapse. In turn, this might also be indicated by the lack of Neuroligin 2 upregulation in the Neuroligin 4deficient retina (Figure 3.18C, D). Thus, an alternative hypothesis is that the upregulation of Neuroligin 4 in the Neuroligin 2-deficient retina relates to a concomitant upregulation of the glycinergic amacrine circuitry, as indicated by the GlyT1 labeling (Figure 3.5D, 3.6D), rather than a direct compensation for a loss of Neuroligin 2.

As Neuroligin 2 is expressed densely at the IPL, and as Neuroligin 4 occupies only a small proportion of synapses at the IPL, one cannot however, exclude the possibility of a subtle upregulation of Neuroligin 2 in the Neuroligin 4-deficient retina. 


\subsection{Information processing in the Neuroligin-deficient retina}

Visual information processing was dramatically impaired in the Neuroligin 2-deficient retina, where the $\mathrm{GABA}_{\mathrm{A}}$ receptor deficiency led to an altered stimulus driven spiking of the ganglion cells (Figure 3.7). A loss of Neuroligin 4, however, led to a subtle impairment in the glycinergic inhibition at the IPL, which did not cause dramatic alterations in the visual coding efficacy of the ganglion cells (Figure 3.13). For time and technical reasons, the role of Neuroligin 3 in visual information processing could not be analyzed in the present study.

\subsubsection{Information processing in the Neuroligin 2-deficient retina}

Quite uniquely throughout the central nervous system, inhibitory transmission in the retina is mediated to similar extents by glycinergic and GABAergic amacrine cells, which modulate visual information as it flows from photoreceptors to ganglion cells. Accordingly, at the IPL, amacrine cells mediate feedback inhibition onto bipolar cell terminals and feedforward inhibition onto ganglion cells, in addition to maintaining serial inhibitory connections among themselves. These effects are mediated by molecularly, functionally and pharmacologically distinct cohorts of postsynaptic receptors (for review see Wassle et al., 1998). For example at the rod bipolar cell terminal (which is central to dim-light vision), inhibitory inputs mediated by $\mathrm{GABA}_{\mathrm{A}}, \mathrm{GABA}_{\mathrm{C}}$ and glycine receptors differentially affect light-evoked signaling and hence rod bipolar cell output, with the GABAergic input being additionally regulated by $\mathrm{GABA}_{\mathrm{A}}$ receptor-mediated inhibition between amacrine cells (Eggers and Lukasiewicz, 2006). At this terminal, inhibition is carried out largely by $\mathrm{GABA}_{\mathrm{C}}$ receptors, which, due to their high sensitivity and relatively sustained response properties, have the potential of exerting fine control over the bipolar cell output (reviewed by Lukasiewicz, 1996). On the other hand, GABAergic inhibition at the level of amacrine and ganglion cells is carried out by transient (fast-deactivating) $\mathrm{GABA}_{\mathrm{A}}$ receptors (reviewed in Lukasiewicz and Shields, 1998). Ganglion cell dendrites express $\mathrm{GABA}_{\mathrm{A}}, \mathrm{GABA}_{\mathrm{B}}$ and glycine receptors (reviewed in Feigenspan and Bormann, 1998; Wassle et al., 1998). Surround inhibition, and hence receptive fields of retinal ganglion cells are directly controlled by GABAergic amacrine cells (via $\mathrm{GABA}_{\mathrm{A}}$ receptors) in the rabbit (Flores-Herr et al., 2001) and salamander (Cook and McReynolds, 1998) retina, which in addition mediate lateral inhibition at the level of bipolar cell terminals at the IPL. Horizontal cell-driven mechanisms at the OPL also contribute indirectly to the shaping of the receptive fields. Taken together GABAergic inhibition has a prominent role in modulating information processing at the IPL. It was thus crucial to evaluate the functional consequences 
of Neuroligin 2 deletion, which affected the clustering of a subset of $\mathrm{GABA}_{\mathrm{A}}$ receptors at the IPL (Figure 3.6). Accordingly, ganglion cell spiking activity was analyzed in Neuroligin 2deficient retina, through multi-electrode recordings.

The relative proportions of ON, OFF and ON-OFF ganglion cells and their average basal firing rate in wild-type paralleled earlier findings (Nirenberg and Meister, 1997; Demas et al., 2003), confirming the validity of the recordings. In particular, the multi-electrode recordings were biased towards the measurement of ON-ganglion cells as described previously (Nirenberg and Meister, 1997). In the Neuroligin $2 \mathrm{KO}$, the distribution of ganglion cell subtypes was unchanged, reflecting the integrity of retinal neuron populations and corroborating the results from the morphological analysis of these animals. Also, ON and OFF $\mathrm{KO}$ cells fired maximally in response to light flashes (Figure 3.7F, I), consistent with an intact excitatory transmission. Neuroligin 2-deficient ganglion cells, however, displayed increased excitability as evident from a significantly higher basal, non-stimulus related, firing rate (Figure 3.7C-E). It is conceivable that the increased excitability results from the altered $\mathrm{GABA}_{\mathrm{A}}$ receptors clustering observed at the IPL, as GABAergic amacrine cells modulate ganglion cell activity (For review, see MacNeil and Masland, 1998). Moreover, an impairment was observed in the coding of light stimuli by Neuroligin $2 \mathrm{KO}$ ganglion cells, as displayed by the reduced peak response to a $1 \mathrm{sec}$ light pulse, when expressed relative to the total number of spikes fired by a given cell (Figure 3.7G-H). Thus, fewer of the spikes received by postsynaptic neurons relate to the light stimulus. One possible reason for this impaired coding might be the "dilution" of light-triggered action potentials by the increased baseline activity of the $\mathrm{KO}$ ganglion cells. In addition, ganglion cell coding might be impaired directly as $\mathrm{GABA}_{\mathrm{A}}$ receptors are missing at the IPL.

The dynamic properties of Neuroligin 2-deficient ganglion cells were additionally analyzed by recording their responses to a "white-noise" (pseudo-random flickering) stimulus (stimulus designed and recordings analyzed by Dr. Bjoern Falkenburger). Subsequently, the $500 \mathrm{msec}$ of stimulus preceding each action potential was averaged for each neuron (spike-triggered average (STA)). This STA represents the "typical" stimulus that evokes an action potential, or the information carried by an average action potential. The STA peak amplitudes were significantly smaller, and the latencies of peaks in the STA significantly shorter, in Neuroligin 2-deficient animals. Shorter latencies of the STAs reflect increased excitability of the Neuroligin 2-deficient ganglion cells, and relate to the impairment of surround inhibition at the IPL of these animals. Reduced STA amplitudes indicate an impaired coding of light, as they imply that the average information per action potential was smaller in the Neuroligin 2 KO. 
In addition, global retinal activity was analyzed in the Neuroligin 2-deficient mice by carrying-out electroretinogram (ERG) recordings (performed by Christian Rudiger, in the department of Prof. Tobias Moser). ERGs analyze the net electrical activity of the retina in response to light flashes of increasing intensity and duration. Principally, the ERG response has three components: the a-wave (attributed to the photoreceptor response), the b-wave (thought to primarily reflect bipolar cell activity), and the oscillatory potentials (representing inhibitory feedback loops at the IPL involving bipolar cell terminals, amacrine cell processes and ganglion cell dendrites) (Wachtmeister, 1998). ERG traces of Neuroligin 2 KO were normal and comparable to WT age-matched littermates, for both the a- and b-waves. This indicates the absence of a general developmental or degenerative defect in Neuroligin $2 \mathrm{KO}$ mice. It also confirms that the main excitatory pathway, phototransduction and signal transmission to the bipolar cell dendrites, remains unperturbed in the absence of Neuroligin 2 . In contrast, a subtle impairment in the amplitude of oscillatory potentials was observed in the absence of Neuroligin 2. At the IPL, inhibitory feedback loops contribute to the generation of these potentials (Wachtmeister, 1998), with a pharmacological disruption of GABA-mediated pathways abolishing oscillations in the mudpuppy retina (Wachtmeister and Dowling, 1978; Wachtmeister, 1980). Consequently, it is likely that Neuroligin 2-driven alteration of oscillatory potentials relate to the deficit in $\mathrm{GABA}_{\mathrm{A}}$ receptor clustering observed at the IPL. The absence of a more severe alteration in inner retinal processing may be due to the fact that a portion of $\mathrm{GABA}_{\mathrm{A}}$ receptors remain functional, as Neuroligin 2 loss impairs, but does not fully abolish, the clustering of specific receptor subsets. Moreover, GABA- and glycinergic amacrine cells are mutually inhibitory i.e., blocking inhibition mediated by GABA often enhances inhibition mediated by glycine and vice-versa (Zhang et al., 1997). As an upregulation of the glycinergic amacrine cells was indicated by the elevated levels of GlyT1 in the Neuroligin $2 \mathrm{KO}$ one needs to evaluate further if there is an enhanced function of the glycinergic circuit in the absence of Neuroligin 2. Finally, the genetic ablation of $\mathrm{GABA}_{\mathrm{C}}$ receptors yields increased oscillatory potential amplitudes (McCall et al., 2002). Accordingly, the slight but not significant reduction in $\mathrm{GABA}_{\mathrm{C}}$ clusters observed at the IPL of Neuroligin 2 KO mice might also preclude large oscillatory potential alterations.

Altogether, both ERG and MEA recordings indicated a significant role of Neuroligin 2 in retinal information processing and correlate well with the morphological analysis of Neuroligin 2-deficient retinae (wherein specific deficits of the amacrine circuitry were unraveled, leaving the core architecture of the retina intact). 
In a final step, higher-order visual processing was evaluated in the absence of Neuroligin 2. Inhibitory interactions at the IPL shape the receptive fields of the ganglion cells and are thus central to the processing of spatial information and contrast function (Cook and McReynolds, 1998; Flores-Herr et al., 2001; Sinclair et al., 2004). Specifically, lateral inhibition mediated by widefield GABAergic amacrine cells has been shown to directly control the centersurround receptive field arrangement and hence spatial processing of retinal ganglion cells (Flores-Herr et al., 2001; Sinclair et al., 2004). In addition, contrast sensitivity in the visual system relies on the center-surround receptive field arrangements of ganglion and bipolar cells, both mediated by feedback GABAergic inhibition (reviewed by Lukasiewicz and Shields, 1998). Thus, the specific deficit in $\mathrm{GABA}_{\mathrm{A}}$ receptor clustering and ganglion cell activity concomitant to a loss of Neuroligin 2 might alter receptive field organization of ganglion cells and hence higher order visual processing. Interestingly, visual acuity and contrast function were assessed in the Neuroligin 2-deficient animals and were found to be dramatically impaired in these animals (experiments performed by Karl-Friedrich Schmidth, in the department of Prof. Siegrid Loewel, Jena, Germany). Of course, higher-order visual areas such as the lateral geniculate nucleus (LGN), superior colliculus, and the visual cortex also rely on GABAergic inhibition (Montero and Zempel, 1986; Fitzpatrick et al., 1987) for information processing. Moreover, $\mathrm{GABA}_{\mathrm{A}}$ receptors have been shown to control surround inhibition, and hence receptive fields, at the superior colliculus, LGN and visual cortex (Sillito, 1974; Sillito and Kemp, 1983; Sillito et al., 1985; Binns and Salt, 1997). Thus, while some of the visual impairment in the Neuroligin 2-deficient animals is certainly attributable to the first order alteration reported in the retina, one cannot exclude the possibility that a loss of Neuroligin 2 affects GABA receptor clustering/integrity also in the higher-order visual areas, thereby compromising visual functions like acuity and contrast. Therefore, it will be interesting in future, to characterize the density and function of GABA receptors at these visual integrative areas in the Neuroligin 2-deficient animals, to acquire a thorough understanding of the role of Neuroligin 2 in the visual system.

In conclusion, the removal of Neuroligin 2 disrupts the integrity of GABAergic postsynapses and leads to impaired information processing by the ganglion cells. This affects the retinal output and has important consequences for the quality of vision in the Neuroligin 2-deficient animals.

Of note, Neuroligin 2 also associates with $\mathrm{GABA}_{\mathrm{A}}$ receptor clusters at the OPL of the retina (Figure 3.2A) however the function of Neuroligin 2 at this synaptic layer of the retina remains unclear. 
Characterization of Neuroligin 2 in different parts of the brain has provided further evidence that Neuroligin 2 controls the organization and function of distinct subsets of inhibitory postsynapses. In the brainstem, Neuroligin 2 is crucial for the activity of both GABA- and glycinergic inhibitory postsynapses (Poulopoulos et al., 2009). In the hippocampus, Neuroligin 2 specifically mediates GABAergic network inhibition (Jedlika et al), as in the dentate gyrus of Neuroligin 2-deficient animals a strong increase in granule cell excitability together with an impairment in the paired-pulse inhibition of the population spike, can be observed. The subsynaptic functional specificity unraveled for the Neuroligin isoforms in the retina, is in agreement to the progressively appearing unsuspected specificity of Neuroligins at different types of synapses, even synaptic inputs. Paired patch-clamp recordings from primary somatosensory cortex revealed that deletion of Neuroligin 2 selectively lowered the strength of synapses of GABAergic Parvalbumin-containing (fast-spiking) interneurons, without any effect on the synapses formed by Somatostatin-containing interneurons (Gibson et al., 2009). Additionally, recent studies have shown that Neuroligin 1 also acts in a subset specific manner to modulate the synaptic plasticity in amygdala circuits, by regulating the abundance of postsynaptic NMDA receptors in an input-specific manner (Jung et al., 2010). Thus, it will be important in the future to examine the Neuroligin mutants in different brain regions to ascertain their synaptic specificities.

\subsubsection{Information processing in the Neuroligin 4-deficient retina}

Although Neuroligin 4 associates in part with different GlyR subsets, morphological investigation of the Neuroligin 4-deficient retina revealed a specific reduction in the $\alpha 1$ subunit-containing GlyR clusters (Figure 3.11). Functionally, the $\alpha 1$ subunit-containing glycinergic synapses have fast decay time constants, as studied in the brainstem (Singer et al., 1998) and in the retina. At the IPL of the mouse retina, OFF-cone bipolar cells, rod bipolar cells and the large A-type ganglion cells all express $\alpha 1$ containing GlyR synapses and display glycine currents with fast decay time constants (Ivanova et al., 2006; Majumdar et al., 2007).

Furthermore, mice lacking the GlyR $\alpha 1$ subunit (Glra $1^{\text {spd-ot }}$ mice) display a significantly reduced frequency of glycinergic spontaneous inhibitory postsynaptic currents (sIPSCs), with the remaining sIPSCs demonstrating radically slower kinetics in comparison to control (mean decay time constant $\tau$ shifted from less than $5 \mathrm{msec}$ in WT to $10 \mathrm{msec}$ in the Glral ${ }^{\text {spd-ot }}$ mice) (Majumdar et al., 2007). In the Neuroligin 4-deficient mice, however, we have a modest reduction of GlyR $\alpha 1$ receptor clusters at the IPL (Figure 3.11). To assess if this small 
reduction in a subset of GlyRs would entail functional consequences, single-cell recordings from retinal ganglion cells were carried out in a whole-mount preparation and both glycinergic and GABAergic mIPSCs were assessed. Recordings were performed in retinal whole-mounts to ensure that the synaptic connectivity of the retina remains intact. From these recordings, about one-third of the ganglion cells displayed glycinergic mIPSCs, which is smaller than the fraction reported in earlier studies, where in retinal slices, half of the ganglion cells were described to receive both GABA- and glycinergic input, with the other half receiving only GABAergic input (Tian et al., 1998).

The glycinergic mIPSCs from Neuroligin $4 \mathrm{KO}$ retinae were unchanged in frequency, reflecting the integrity of the glycinergic presynaptic compartment (Figure 3.12C). However, they were slightly smaller in amplitude, displayed a slower $20-80 \%$ rise time, and were significantly slower in their decay time course (decay time constant was $\sim 18 \%$ slower than the WT controls), indicating that Neuroligin 4 deletion exerts an effect on the amplitude and kinetics of glycinergic mIPSCs (Figure 3.12C, D). In contrast, GABAergic mIPSCs recorded from Neuroligin 4-deficient ganglion cells were identical to wild-type in all parameters assessed, illustrating the validity of the recordings (Figure 3.12E).

The lack of a dramatic impairment in glycinergic mIPSCs subsequent to the loss of Neuroligin 4 may be explained by the fact that Neuroligin 4 equips only $\sim 21 \%$ of glycinergic postsynapses in the retina and a loss of Neuroligin 4 leads to a subtle $(16.7 \%)$ reduction in a specific GlyR subset. However, in congruence with the morphological data, the functional alterations observed in the glycinergic mIPSCs from Neuroligin 4-deficient ganglion cells do indicate an impairment of a subset of glycinergic postsynapses reflecting either a reduction in the number of GlyR clusters responsible for kinetically fast responses or a deficit in the postsynaptic clustering of individual GlyR subunits. As the integrity of GlyR subunit clustering has not yet been investigated ultrastructurally in the Neuroligin $4 \mathrm{KO}$ mice, the only explanation that can be currently offered is that GlyRs with fast kinetics are selectively lost in the absence of Neuroligin 4, leading to an alteration in the kinetics of the mIPSCs.

During electrophysiological recordings, stringent criteria were imposed to differentiate between ganglion cells and displaced amacrine cells that are also located at the ganglion cell layer (see 2.7). However, as the selection of ganglion cells was biased towards large ganglion cells that had a diameter above $15 \mu \mathrm{m}$, it is possible that several A-type ganglion cells were recorded from, which predominantly receive kinetically fast glycinergic inputs, by GlyRs composed of $\alpha 1$ and $\beta$ subunits (Majumdar et al., 2007). On the other hand, the proportion of 
ganglion cells that displayed glycinergic mIPSCs in our recordings was not higher than previous estimates, which indicates that the A-type ganglion cells were not oversampled during the electrophysiological recordings.

Only few other studies have analyzed the kinetics of glycinergic mIPSCs from ganglion cells in retinal whole-mounts. The decay time constant of glycinergic sIPSCs from A-type ganglion cells has been estimated as $\tau=3.9 \pm 2.5 \mathrm{msec}$ (mean $\pm \mathrm{SD}$ ) (Majumdar et al., 2007). However, these recordings were carried out at a holding potential of $-5 \mathrm{mV}$, and the decay time course of glycinergic IPSCs has been shown to be voltage dependent with a slower decay at more positive membrane potentials (Smith et al., 2000; Gill et al., 2006). Hence, for the recordings performed at $-70 \mathrm{mV}$, the decay time constant is expected to be $\sim 67 \%$ of the value measured at $-5 \mathrm{mV}$. This is close to the value estimated in the present study for the decay time constant of glycinergic mIPSCs from WT ganglion cells $(\tau=2.42 \pm 0.0965 \mathrm{msec})$ (mean \pm SEM). Furthermore, when glycinergic mIPSCs were recorded from WT ganglion cells at depolarized potentials, the decay $\tau$ was prolonged (at $\mathrm{V}_{\text {holding }}=-50 \mathrm{mV}, \tau \sim 2.74 \mathrm{msec}$, and at $\mathrm{V}_{\text {holding }}=-30 \mathrm{mV}, \tau \sim 3.01 \mathrm{msec}$ ), confirming the voltage dependence of the mIPSC decay kinetics, as well as the validity of our measurements.

In the retina, the impairment in inhibitory processing in the absence of Neuroligin 4 is subtle. This is in agreement with previous analyses of the Neuroligin 4-deficient mice, which display very mild morphological changes in brain size (1.5\% reduction of total brain volume) but otherwise no alterations in brain cytoarchitecture (Jamain et al., 2008). However, our data are the first to uncover the link of Neuroligin 4 with GlyRs, and these receptor populations should now be looked at in the brain of the Neuroligin $4 \mathrm{KO}$. In any case the specific deficit in fast glycinergic transmission observed in the retina of Neuroligin 4-deficient animals is fascinating, as it may result in an imbalance in excitatory/inhibitory synapse function, a feature frequently associated with autism (Rubenstein and Merzenich, 2003). Moreover, Rubenstein and Merzenich propose that the dysfunction in autism results from a shift towards excitation (Rubenstein and Merzenich, 2003), which would be the case if inhibition were selectively impaired in the Neuroligin 4-deficient animals.

To assess if the subtle impairment in inhibition affects the information coding capabilities of Neuroligin 4-deficient ganglion cells, multi-electrode recordings were carried out. At first, simple light flashes were used to study the baseline (non-stimulus related) and ON/OFF activity of the Neuroligin 4-deficient ganglion cells. No impairment was uncovered in either the baseline or maximal peak responses of the KO ganglion cells (Figure 3.13B). This 
indicates that both the main excitatory pathway and "background" inhibition at the IPL, which sets the baseline for the ganglion cells, are intact.

In the case of Neuroligin 2 deficiency, an increased baseline firing reflected a decreased steady-state, background inhibition (Figure 3.7). With a loss of Neuroligin 4, however, a subtle deficit in GlyR transmission with fast kinetics was uncovered, which shapes the fast response to light in (A-type) ganglion cells (Majumdar et al., 2007). As the GlyRs responsible for fast neurotransmission do not mediate steady-state inhibition, the lack of change in baseline activity in Neuroligin 4-deficient ganglion cells is not surprising.

However, an alteration in the "fast" inhibitory input onto ganglion cells would be expected to impact on their response to visual stimuli. Upon application of the "white-noise" stimulus paradigm (stimulus programmed and recordings analyzed by Dr. Bjoern Falkenburger), a specific deficit was uncovered in the stimulus-coding efficacy of Neuroligin 4-deficient ganglion cells. An imbalance was observed in the Neuroligin 4-deficient retinal ganglion cells, such that more spikes were coding for the stimulus. Furthermore, the Neuroligin 4-deficient ganglion cells displayed a shallow maximal response slope as compared to WT control. Thus, a loss of "fast" feedback inhibition onto Neuroligin 4-deficient ganglion cells leads to an alteration in the proportion of stimulus-related spikes, which could underlie a decreased spatial and temporal precision of information processing. Taken together, the impairment in the inhibitory drive onto the ganglion cells, although subtle, had clearly detectable consequences for visual information coding, and correlates well with the aforementioned morphological and electrophysiological alterations in the Neuroligin 4-deficient retina.

As the coding of visual information was not drastically altered in the absence of Neuroligin 4, we wanted to know whether it might suffice to perturb higher-order visual activities. To address this question, visual acuity and contrast sensitivity were measured in the Neuroligin 4deficient animals. These tests were particularly exciting to carry out in the Neuroligin 4deficient animals, as visual and retinal disturbances have been observed in a subset of autistic patients (reviewed in Dakin and Firth, 2005; Behrmann et al., 2006). In particular, a study by Ashwin et al., (2009) has reported a significantly improved visual acuity in a subset of patients with ASDs as compared to controls. However, both visual acuity and contrast sensitivity remained unchanged in the Neuroligin $4 \mathrm{KO}$ mice, indicating that the integrative function of higher-order visual centers remains unperturbed upon the loss of Neuroligin 4 (experiments performed by Karl-Friedrich Schmidth, in the department of Prof. Siegrid Loewel, Jena, Germany). This could be due to the fact that processing at these cortical centers relies heavily on GABAergic and not glycinergic interneuronal activity (Montero and Zempel, 1986; 
Fitzpatrick et al., 1987). Nevertheless, more precise measurements would be necessary to rule out the presence of a subtle impairment in information processing at higher visual centers in the Neuroligin 4-deficient animals.

The differential effects of each Neuroligin isoform on visual processing are striking. As discussed in 4.4.1, the impairment of GABA driven inhibition at the IPL of Neuroligin 2deficient animals leads to a dramatic reduction of visual acuity and contrast function in these animals. This would be expected as lateral inhibition mediated by widefield GABAergic amacrine cells has been shown to control the center-surround receptive field arrangement and hence spatial processing of retinal ganglion cells (Flores-Herr et al., 2001; Sinclair et al., 2004). The fact that Neuroligin 4-deficient animals, with a selective impairment of the fast glycinergic transmission, show no perturbation of higher order visual processing could mean that small field glycinergic amacrine cells are not vital for the spatial tuning of ganglion cells. Alternatively, the impairment observed for the Neuroligin 4-deficient ganglion cells is not significant to cause a strong effect on visual processing of contrast and acuity.

Recent studies have analyzed global retinal activity in the Neuroligin 4-deficient animals. A dramatic impairment was observed in the b-wave amplitude (experiments performed by Christian Rudiger, in the department of Prof. Tobias Moser), subsequent to Neuroligin 4 deletion. This is puzzling as the b-wave is thought to represent bipolar cell-driven processing in the retina, in particular the transmission from photoreceptor terminals at the OPL to bipolar cell dendrites. As Neuroligin 4 was not expressed at the OPL, the only possible explanation, for this finding would be that inhibitory feedback onto bipolar cell terminals is somehow impaired in the absence of Neuroligin 4. Future in situ hybridization experiments for Neuroligin 4 are needed to clarify the expression of Neuroligin 4 from bipolar cell dendrites at the OPL. Further, electrophysiological experiments probing the efficiency of the amacrine-tobipolar circuit in the IPL would be necessary to ascertain the glycinergic input onto Neuroligin 4-deficient bipolar cell terminals. Investigating this bipolar cell deficit will be of added interest since, the b-wave amplitudes are reduced in a subset of autistic patients (Ritvo et al., 1988).

In conclusion, Neuroligin 4 is localized at a subset of glycinergic postsynapses in the retina, and a loss of Neuroligin 4 is accompanied with a subtle deficit in fast glycinergic transmission, which modulates information processing by retinal ganglion cells.

Recent Neuroligin 4 localization studies in the brainstem and spinal cord have revealed a specific distribution of Neuroligin 4 at GlyR-bearing postsynapses (M. Hammer and F. Varoqueaux, unpublished observations). Glycinergic neurotransmission plays an important 
role in modulating activity in both of these regions of the CNS (Zafra et al., 1997; reviewed in Legendre, 2001; Kirsch, 2006). Glycinergic interneurons control motor rhythm generation underlying locomotion (for review see Legendre, 2001), in addition to mediating processing of sensory information that governs vision, audition (Lopez-Corcuera et al., 2001) and inflammatory pain sensitization (Harvey et al., 2004). In the adult rat CNS, $50 \%$ of neurons in most brainstem nuclei show GlyR immunoreactivity (Liu and Wong-Riley, 2005), and in the lateral superior olive (part of the brainstem that is crucial for auditory processing) there is a developmental transition from GABA- to glycinergic neurotransmission during the first two weeks of life (Kotak et al., 1998). Thus, glycinergic transmission plays an important role in modulating information processing at both the brainstem and spinal cord. Consequently it will be important in the future, to study the role of Neuroligin 4 in inhibitory processing in these regions of the CNS by analysis of the Neuroligin 4-deficient mice. However, the Neuroligin 4deficient mice show no signs of motor deficits (Jamain et al., 2008) and it should be kept in mind that Neuroligin 2 is also present at the inhibitory synapses in the brainstem (Poulopoulos et al., 2009) where GABA is essentially co-released with glycine to act upon a mixed population of GABA and glycine receptors located at these synapses (O'Brien and Berger, 1999; Awatramani et al., 2005). Thus it might be difficult to unravel functional deficits in these regions of the Neuroligin 4 deficient animals.

\subsubsection{Functional consequences of Neuroligin 3 deletion}

Loss-of-function mutation in Neuroligin 3 has been identified in a subset of autistic patients (Jamain et al., 2003). This mutation leads to a trafficking deficit, whereby $\sim 90 \%$ of the protein is retained at the ER (Comoletti et al., 2004; Tabuchi et al., 2007). Accordingly, the Neuroligin 3 knockout mouse has recently been introduced as a genetic model for the study of autism (Radyushkin et al., 2009). In another study the analysis of excitatory and inhibitory evoked responses in the somatosensory cortex of Neuroligin $3 \mathrm{KO}$ mice revealed a subtle (not significant) reduction in the strength of excitatory evoked responses, with no alterations in the inhibitory responses (Tabuchi et al., 2007). Morphologically, this was paralleled by an intact synapse density (both excitatory and inhibitory) at the hippocampus and barrel cortex of Neuroligin 3-deficient brain sections. Thus, the only analysis done so far on the Neuroligin 3 $\mathrm{KO}$ mouse has not revealed a distinct role of Neuroligin 3 at the synapse.

In the retina, however, deficiency of Neuroligin 3 led to a dramatic reduction in $\alpha 2$-subunit containing $\mathrm{GABA}_{\mathrm{A}}$ receptors, at the IPL (Figure 3.16). This may imply functional consequences for information processing by retinal ganglion cells. Moreover, even though the 
rest of the GABA-/glycinergic synapses in the IPL seem to be unaltered morphologically, they might nevertheless have functional deficits, which could affect information processing. Thus, the functional analysis of Neuroligin 3-deficient mice is crucial.

Interestingly, genes controlling GABA receptors have been implicated in ASDs (Ma et al., 2005) and post-mortem imaging and histological studies have shown that neurons from autistic patients exhibit a decrease in GABA production, neurite branching and arborization (Kemper and Bauman, 2002; Zoghbi, 2003; Bauman and Kemper, 2005). Further, electrophysiological recordings from hippocampal neurons in WT and autism associated Neuroligin 3 (R471C) mutants have shown a reduction of inhibitory activity in networks carrying the mutation in comparison to networks overexpressing wild-type Neuroligin 3 (Gutierrez et al., 2009). Further, spontaneous network activity patterns as analyzed by optical techniques revealed that the autism-associated Neuroligin 3 mutation affected network synchrony (Gutierrez et al., 2009), a form of correlated neuronal activity, controlled by the coordination of inhibitory and excitatory synaptic events (Salinas and Sejnowski, 2001). All these studies, underscore the importance of evaluating network activity (inhibitory in particular) in Neuroligin 3-deficient mutants, in the retina as in different areas of the brain.

Retinal processing in the Neuroligin 3-deficient animals is currently being investigated. Initial studies analyzing ganglion cell spiking activity by multi-electrode recordings have unraveled a rather surprising finding: ON-ganglion cells were recorded in a greater proportion at the expense of OFF ganglion cells in the Neuroligin 3-deficient retinae. This indicates an imbalance in the occurrence (development or maturation) of the different ganglion cell subtypes (T. Gollisch unpublished observations). These ganglion cell subsets now need to be morphologically investigated to corroborate the findings from the multi-electrode recordings. The multi-electrode analysis of Neuroligin 3-deficient retina highlights the importance of Neuroligin 3 in mediating the differentiation of retinal ganglion subsets that are functionally distinct and make connections at specified strata within the IPL. The morphological investigation of Neuroligin 3-deficient retinae has so far only pointed to the loss of a subset of $\mathrm{GABA}_{\mathrm{A}}$ receptors. This GABAergic deficit might lead to an alteration in ganglion cell differentiation, as GABAergic inhibition modulates the "bursting" activity of the ganglion cells, which occurs at a time when ON/OFF characteristics are specified (Wang et al., 2007; reviewed in Wong, 1999). In this regard it might also be interesting to look at the development of the Neuroligin 3-deficient retina. 
In summary, Neuroligin 3 is localized at a majority of inhibitory (a 3:2 ratio between $\mathrm{GABA}_{\mathrm{A}^{-}}$ and glycinergic) postsynapses, and a loss of Neuroligin 3 is detrimental to the clustering of a subset of $\mathrm{GABA}_{\mathrm{A}}$ receptors. This impairment in a subclass of GABA receptors, might alter the information processing abilities of the retina, or even modulate the development of ganglion cell specificities. These issues are currently being investigated. 


\subsection{Neuroligins and sensory processing}

The characterization of Neuroligin-deficient retinae in this study has highlighted important contributions of each Neuroligin isoforms to visual information processing. Neuroligin 2 modulates the GABAergic inhibition at the IPL, and thus is instrumental in controlling the efficiency of visual information coding by ganglion cells. Neuroligin 4 on the other hand, modulates the fast glycinergic inhibition onto ganglion cells, which shapes their responses to visual stimuli. In addition, morphological data indicate that Neuroligin 3 may also play an instrumental role in maintaining inhibitory surround at the IPL. Thus, in the mouse retina, Neuroligin isoforms participate in maintaining the integrity of the inhibitory postsynaptic compartment and hence, information processing.

Recent work done in other species has also demonstrated a role of Neuroligins in sensory information processing. Studies in the honeybee brain have shown that Neuroligins can be differentially regulated by sensory input (Biswas et al., 2010). Honeybees have been reported to express all the human Neuroligin variants (Neuroligin 1-5) (Biswas et al., 2008) and sensory deprivation leads to a downregulation of Neuroligin 1 expression, together with an increased expression of Neuroligins 2-5. Moreover, bees that have undergone associative scent training show increased levels of Neuroligin 1 and 3 as compared to untrained controls. These results demonstrate that Neuroligin expression can be modulated by sensory stimulation. In addition, Neuroligins have been implicated in sensory processing in C. elegans, which contains a single Neuroligin 1 gene, expressed in sensory neurons, interneurons and a subset of cholinergic motor neurons (Hunter et al., 2010). Interestingly, Neuroligin 1-deficient mutants are viable, without any motor deficits, comparable to Neuroligin 1 KO mice (Varoqueaux et al., 2006; Blundell et al., 2010). However, the C. elegans mutants displayed deficits in the processing of chemosensory cues (Hunter et al., 2010). Furthermore, Neuroligins are also expressed in the nervous system of zebrafish, with Neuroligin 2, Neuroligin 3, and Neuroligin 4 being duplicated, but Neuroligin 1 having a single zebrafish ortholog (Davey et al., 2010). Future studies will reveal the role of these diverse Neuroligin isoforms in the development and function of the zebrafish nervous system.

In summary, the importance of Neuroligins in sensory processing is just beginning to get appreciated, with researchers employing diverse model systems to investigate the role of Neuroligins in the processing of different sensory systems. 


\section{Summary}

Neuroligins are postsynaptic cell adhesion proteins instrumental for synapse maturation and function. This study analyzed the distribution of Neuroligin 2, 3 and 4 in the well-structured synaptic network of the retina. The role of individual Neuroligin isoforms in the maintenance of the retinal circuitry was studied by analyzing the synaptic organization of the isoformspecific deletion mutant mice. In addition, functional studies were performed to characterize the contribution of Neuroligin 2 and 4 in the maintenance of network activity and visual information processing.

Neuroligin 2 is localized preferentially at GABAergic postsynapses of both synaptic layers of the retina. Loss of Neuroligin 2 is accompanied by a dramatic reduction in specific subsets of $\mathrm{GABA}_{\mathrm{A}}$ receptors (those containing the $\alpha 3$ and $\gamma 2$ were dramatically reduced in density), together with an upregulation of the glycinergic amacrine circuitry. The consequent impairment in the steady-state inhibition at the inner synaptic layer impairs the stimuluscoding efficacy of retinal ganglion cells and also affects higher-order visual processing. Instead, Neuroligin 4 localizes selectively to glycinergic postsynapses of the retina. The expression of Neuroligin 4 in the retina is not as robust as that of the other Neuroligin isoforms, and Neuroligin 4 occupies only a subset of GlyR postsynapses. Accordingly, a loss of Neuroligin 4 leads to a small reduction in the density of GlyR clusters that are responsible for kinetically fast transmission. As a result, the Neuroligin 4-deficient ganglion cells display glycinergic mIPSCs with significantly slower decay time constants and slightly smaller amplitudes. These subtle modulations of glycinergic transmission at the IPL did not drastically perturb the information-coding abilities of the ganglion cells or higher-order visual processing. Neuroligin 3 is also associated with inhibitory postsynapses in the retina (3:2 ratio between $\mathrm{GABA}_{\mathrm{A}^{-}}$and glycinergic). A loss of Neuroligin 3 is paralleled by a selective reduction in the density of $\mathrm{GABA}_{\mathrm{A}}$ receptor subsets containing the $\alpha 2$ subunit. The functional consequences of this perturbation on the visual processing capabilities of retinal ganglion cells are currently being investigated.

In essence, this study is the first characterization of Neuroligin distribution and function in the retina, and reveals interesting diverse roles of the different Neuroligin isoforms in visual information processing. 


\section{Acknowledgments}

At first I would like to thank my PhD advisor: Dr. Frederique Varoqueaux. The journey of my $\mathrm{PhD}$ would have never been so educative, enjoyable and wholesome had it not been for her tireless supervision. She introduced me to the wonderful world of retinal research for which I will always remain grateful.

I am also indebted to Prof. Dr. Nils Brose for including me as a member in his department. It has been an enriching experience to be a part of his department and his guidance and input to my $\mathrm{PhD}$ project has been precious. I am also truly grateful to the guidance and effort of my other thesis committee members: Prof. Tobias Moser and Dr. Joachim Bormann.

I am grateful to the IMPRS $\mathrm{MS} / \mathrm{PhD}$ Neuroscience program, for providing me with the opportunity to experience the scientific atmosphere here in Goettingen. I would specially like to thank the program coordinators: Prof. Dr. Michael Hoerner and Ms. Sandra Drube for their continuous help and support over all these years.

I would also like to express my gratitude to my $\mathrm{PhD}$ training program NEUREST. In particular the NEUREST program coordinators: Dr. Joachim Bormann, Ms. Eva Strehler, Dr. Alexander Zimek and Dr. Thomas Fritzsche. Further, I would like to mention the Goettingen GGNB graduate program and the Center for Molecular Physiology in the brain (CMPB) for funding the last months of my $\mathrm{PhD}$.

The interactive scientific environment in Goettingen enabled several collaborations to learn techniques, which were invaluable tools for the progress of my $\mathrm{PhD}$ project. These collaborations also gave me an opportunity to interact with highly experienced scientists like Dr. Bjoern Falkenburger, with whose help we set-up the multi-electrode recordings for the retina and who was invaluable for the analysis of these recordings and Dr. Holger Taschenberger, who was kind and helpful in allowing his experimental set-up to be used for whole-mount recordings in the retina, and who was instrumental for the analysis of these single-cell recordings.

I would also like to thank Prof. Heinz Wassle, Prof. Rachel Wong, Dr. Josef Ammermuller, Dr. Tim Gollisch, Dr. Silke Haverkamp, Dr Michiel Van Wyk and Dr Karin Dedek for precious advice during early stages of the study.

Further, I would like to acknowledge the support of all members of the molecular neurobiology department throughout the course of my PhD. Specially the support of Ramya, Rieke, Venita, Ben, Alexandra, Matthieu, Alexandros, Tolga, Cordelia, Christoph, Klaus, Fritz 
and Martin. I am also appreciative of the support of Kun-Han, Chao-Hua and Raunak from the department of membrane biophysics. I had the privilege of supervising Helena Magliarelli as my Lab rotation student, and I would like to acknowledge her support for the characterization of Neuroligin 3 in the mouse retina.

I am also deeply appreciative of the emotional support of my friends. In particular, Raunak, for being a extremely patient listener and for encouraging me in science, sports and life... Finally and most importantly, I am grateful to my parents and my sister who have supported me immensely, despite being so far away. 


\section{References}

Ahmari SE, Buchanan J, Smith SJ (2000) Assembly of presynaptic active zones from cytoplasmic transport packets. Nat Neurosci 3:445-451.

Alldred MJ, Mulder-Rosi J, Lingenfelter SE, Chen G, Luscher B (2005) Distinct gamma2 subunit domains mediate clustering and synaptic function of postsynaptic GABAA receptors and gephyrin. J Neurosci 25:594-603.

Allison DW, Chervin AS, Gelfand VI, Craig AM (2000) Postsynaptic scaffolds of excitatory and inhibitory synapses in hippocampal neurons: maintenance of core components independent of actin filaments and microtubules. J Neurosci 20:4545-4554.

Amaral D, Lavenex P (2006) Hippocampal Neuroanatomy in 'The Hippocampus Book' edited by Andersen P, Morris R, Amaral D, Bliss T, O'Keefe J: Oxford University Press.

Ashwin E, Ashwin C, Rhydderch D, Howells J, Baron-Cohen S (2009) Eagle-eyed visual acuity: an experimental investigation of enhanced perception in autism. Biol Psychiatry 65:17-21.

Association AP (1994) Diagnostic and Statistical Manual of Mental Disorders (4th edn). Washington, D.C.: American Psychiatric Press.

Awatramani GB, Turecek R, Trussell LO (2005) Staggered development of GABAergic and glycinergic transmission in the MNTB. J Neurophysiol 93:819-828.

Baer K, Essrich C, Benson JA, Benke D, Bluethmann H, Fritschy JM, Luscher B (1999) Postsynaptic clustering of gamma-aminobutyric acid type A receptors by the gamma3 subunit in vivo. Proc Natl Acad Sci U S A 96:12860-12865.

Bauman ML, Kemper TL (2005) Neuroanatomic observations of the brain in autism: a review and future directions. Int J Dev Neurosci 23:183-187.

Behrmann M, Thomas C, Humphreys K (2006) Seeing it differently: visual processing in autism. Trends Cogn Sci 10:258-264.

Berntson A, Taylor WR (2000) Response characteristics and receptive field widths of on-bipolar cells in the mouse retina. J Physiol 524 Pt 3:879-889.

Berry MJ, Warland DK, Meister M (1997) The structure and precision of retinal spike trains. Proc Natl Acad Sci U S A 94:5411-5416.

Betz H (1991) Glycine receptors: heterogeneous and widespread in the mammalian brain. Trends Neurosci 14:458-461.

Biederer T, Sara Y, Mozhayeva M, Atasoy D, Liu X, Kavalali ET, Sudhof TC (2002) SynCAM, a synaptic adhesion molecule that drives synapse assembly. Science 297:1525-1531.

Billups D, Hanley JG, Orme M, Attwell D, Moss SJ (2000) GABAC receptor sensitivity is modulated by interaction with MAP1B. J Neurosci 20:8643-8650.

Binns KE, Salt TE (1997) Different roles for GABAA and GABAB receptors in visual processing in the rat superior colliculus. J Physiol 504 ( Pt 3):629-639.

Biswas S, Reinhard J, Oakeshott J, Russell R, Srinivasan MV, Claudianos C (2010) Sensory regulation of neuroligins and neurexin I in the honeybee brain. PLoS One 5:e9133.

Biswas S, Russell RJ, Jackson CJ, Vidovic M, Ganeshina O, Oakeshott JG, Claudianos C (2008) Bridging the synaptic gap: neuroligins and neurexin I in Apis mellifera. PLoS One 3:e3542.

Blundell J, Tabuchi K, Bolliger MF, Blaiss CA, Brose N, Liu X, Sudhof TC, Powell CM (2009) Increased anxiety-like behavior in mice lacking the inhibitory synapse cell adhesion molecule neuroligin 2. Genes Brain Behav 8:114-126.

Blundell J, Blaiss CA, Etherton MR, Espinosa F, Tabuchi K, Walz C, Bolliger MF, Sudhof TC, Powell CM (2010) Neuroligin-1 deletion results in impaired spatial memory and increased repetitive behavior. J Neurosci 30:2115-2129.

Boucard AA, Chubykin AA, Comoletti D, Taylor P, Sudhof TC (2005) A splice code for trans-synaptic cell adhesion mediated by binding of neuroligin 1 to alpha- and beta-neurexins. Neuron 48:229-236.

Bourgeron T (2009) A synaptic trek to autism. Curr Opin Neurobiol 19:231-234.

Boycott B, Wassle $\mathrm{H}$ (1999) Parallel processing in the mammalian retina: the Proctor Lecture. Invest Ophthalmol Vis Sci 40:1313-1327.

Brandstatter JH, Koulen P, Wassle H (1997) Selective synaptic distribution of kainate receptor subunits in the two plexiform layers of the rat retina. J Neurosci 17:9298-9307. 
Brandstatter JH, Fletcher EL, Garner CC, Gundelfinger ED, Wassle H (1999) Differential expression of the presynaptic cytomatrix protein bassoon among ribbon synapses in the mammalian retina. Eur J Neurosci 11:3683-3693.

Bresler T, Shapira M, Boeckers T, Dresbach T, Futter M, Garner CC, Rosenblum K, Gundelfinger ED, Ziv NE (2004) Postsynaptic density assembly is fundamentally different from presynaptic active zone assembly. J Neurosci 24:1507-1520.

Budreck EC, Scheiffele P (2007) Neuroligin-3 is a neuronal adhesion protein at GABAergic and glutamatergic synapses. Eur J Neurosci 26:1738-1748.

Butz S, Okamoto M, Sudhof TC (1998) A tripartite protein complex with the potential to couple synaptic vesicle exocytosis to cell adhesion in brain. Cell 94:773-782.

Cantallops I, Cline HT (2000) Synapse formation: if it looks like a duck and quacks like a duck. Curr Biol 10:R620-623.

Carter-Dawson LD, LaVail MM (1979) Rods and cones in the mouse retina. I. Structural analysis using light and electron microscopy. J Comp Neurol 188:245-262.

Casini G, Brecha NC, Bosco L, Rickman DW (2000) Developmental expression of neurokinin-1 and neurokinin-3 receptors in the rat retina. J Comp Neurol 421:275-287.

Chen L, Chetkovich DM, Petralia RS, Sweeney NT, Kawasaki Y, Wenthold RJ, Bredt DS, Nicoll RA (2000) Stargazin regulates synaptic targeting of AMPA receptors by two distinct mechanisms. Nature 408:936-943.

Chichilnisky EJ (2001) A simple white noise analysis of neuronal light responses. Network 12:199-213.

Chichilnisky EJ, Kalmar RS (2002) Functional asymmetries in ON and OFF ganglion cells of primate retina. J Neurosci 22:2737-2747.

Chih B, Engelman H, Scheiffele P (2005) Control of excitatory and inhibitory synapse formation by neuroligins. Science 307:1324-1328.

Chih B, Gollan L, Scheiffele P (2006) Alternative splicing controls selective trans-synaptic interactions of the neuroligin-neurexin complex. Neuron 51:171-178.

Chih B, Afridi SK, Clark L, Scheiffele P (2004) Disorder-associated mutations lead to functional inactivation of neuroligins. Hum Mol Genet 13:1471-1477.

Chubykin AA, Liu X, Comoletti D, Tsigelny I, Taylor P, Sudhof TC (2005) Dissection of synapse induction by neuroligins: effect of a neuroligin mutation associated with autism. J Biol Chem 280:22365-22374.

Chubykin AA, Atasoy D, Etherton MR, Brose N, Kavalali ET, Gibson JR, Sudhof TC (2007) Activitydependent validation of excitatory versus inhibitory synapses by neuroligin-1 versus neuroligin-2. Neuron 54:919-931.

Clements JD, Bekkers JM (1997) Detection of spontaneous synaptic events with an optimally scaled template. Biophys J 73:220-229.

Comoletti D, Flynn R, Jennings LL, Chubykin A, Matsumura T, Hasegawa H, Sudhof TC, Taylor P (2003) Characterization of the interaction of a recombinant soluble neuroligin-1 with neurexin1beta. J Biol Chem 278:50497-50505.

Comoletti D, De Jaco A, Jennings LL, Flynn RE, Gaietta G, Tsigelny I, Ellisman MH, Taylor P (2004) The Arg451Cys-neuroligin-3 mutation associated with autism reveals a defect in protein processing. J Neurosci 24:4889-4893.

Comoletti D, Flynn RE, Boucard AA, Demeler B, Schirf V, Shi J, Jennings LL, Newlin HR, Sudhof TC, Taylor $P$ (2006) Gene selection, alternative splicing, and post-translational processing regulate neuroligin selectivity for beta-neurexins. Biochemistry 45:12816-12827.

Cook PB, McReynolds JS (1998) Lateral inhibition in the inner retina is important for spatial tuning of ganglion cells. Nat Neurosci 1:714-719.

Couteaux R, Pecot-Dechavassine M (1970) [Synaptic vesicles and pouches at the level of "active zones" of the neuromuscular junction]. C R Acad Sci Hebd Seances Acad Sci D 271:23462349.

Craig AM, Kang Y (2007) Neurexin-neuroligin signaling in synapse development. Curr Opin Neurobiol 17:43-52.

Craig AM, Graf ER, Linhoff MW (2006) How to build a central synapse: clues from cell culture. Trends Neurosci 29:8-20.

Cueva JG, Haverkamp S, Reimer RJ, Edwards R, Wassle H, Brecha NC (2002) Vesicular gammaaminobutyric acid transporter expression in amacrine and horizontal cells. J Comp Neurol 445:227-237.

Cutting GR, Lu L, O'Hara BF, Kasch LM, Montrose-Rafizadeh C, Donovan DM, Shimada S, Antonarakis SE, Guggino WB, Uhl GR, et al. (1991) Cloning of the gamma-aminobutyric acid 
(GABA) rho 1 cDNA: a GABA receptor subunit highly expressed in the retina. Proc Natl Acad Sci U S A 88:2673-2677.

Dakin S, Frith U (2005) Vagaries of visual perception in autism. Neuron 48:497-507.

Davey C, Tallafuss A, Washbourne P (2010) Differential expression of neuroligin genes in the nervous system of zebrafish. Dev Dyn 239:703-714.

Dean C, Dresbach T (2006) Neuroligins and neurexins: linking cell adhesion, synapse formation and cognitive function. Trends Neurosci 29:21-29.

Dean C, Scholl FG, Choih J, DeMaria S, Berger J, Isacoff E, Scheiffele P (2003) Neurexin mediates the assembly of presynaptic terminals. Nat Neurosci 6:708-716.

Demas J, Eglen SJ, Wong RO (2003) Developmental loss of synchronous spontaneous activity in the mouse retina is independent of visual experience. J Neurosci 23:2851-2860.

DeVries SH, Baylor DA (1995) An alternative pathway for signal flow from rod photoreceptors to ganglion cells in mammalian retina. Proc Natl Acad Sci U S A 92:10658-10662.

Di Garbo A (2008) The electrical coupling confers to a network of interneurons the ability of transmitting excitatory inputs with high temporal precision. Brain Res 1225:47-56.

Dick O, Hack I, Altrock WD, Garner CC, Gundelfinger ED, Brandstatter JH (2001) Localization of the presynaptic cytomatrix protein Piccolo at ribbon and conventional synapses in the rat retina: comparison with Bassoon. J Comp Neurol 439:224-234.

Dick O, tom Dieck S, Altrock WD, Ammermuller J, Weiler R, Garner CC, Gundelfinger ED, Brandstatter JH (2003) The presynaptic active zone protein bassoon is essential for photoreceptor ribbon synapse formation in the retina. Neuron 37:775-786.

Dityatev A, El-Husseini A (2006) Molecular Mechanisms of Synaptogenesis Springer.

Dong CJ, Werblin FS (1998) Temporal contrast enhancement via GABAC feedback at bipolar terminals in the tiger salamander retina. J Neurophysiol 79:2171-2180.

Dong N, Qi J, Chen G (2007) Molecular reconstitution of functional GABAergic synapses with expression of neuroligin-2 and GABAA receptors. Mol Cell Neurosci 35:14-23.

Dowling JE, Boycott BB (1966) Organization of the primate retina: electron microscopy. Proc R Soc Lond B Biol Sci 166:80-111.

Durand CM, Betancur C, Boeckers TM, Bockmann J, Chaste P, Fauchereau F, Nygren G, Rastam M, Gillberg IC, Anckarsater H, Sponheim E, Goubran-Botros H, Delorme R, Chabane N, MourenSimeoni MC, de Mas P, Bieth E, Roge B, Heron D, Burglen L, Gillberg C, Leboyer M, Bourgeron T (2007) Mutations in the gene encoding the synaptic scaffolding protein SHANK3 are associated with autism spectrum disorders. Nat Genet 39:25-27.

Eggers ED, Lukasiewicz PD (2006) GABA(A), GABA(C) and glycine receptor-mediated inhibition differentially affects light-evoked signalling from mouse retinal rod bipolar cells. J Physiol 572:215-225.

Enz R, Brandstatter JH, Wassle H, Bormann J (1996) Immunocytochemical localization of the GABAc receptor rho subunits in the mammalian retina. J Neurosci 16:4479-4490.

Essrich C, Lorez M, Benson JA, Fritschy JM, Luscher B (1998) Postsynaptic clustering of major GABAA receptor subtypes requires the gamma 2 subunit and gephyrin. Nat Neurosci 1:563571.

Euler T, Masland RH (2000) Light-evoked responses of bipolar cells in a mammalian retina. J Neurophysiol 83:1817-1829.

Euler T, Schneider H, Wassle H (1996) Glutamate responses of bipolar cells in a slice preparation of the rat retina. J Neurosci 16:2934-2944.

Fairhall AL, Burlingame CA, Narasimhan R, Harris RA, Puchalla JL, Berry MJ, 2nd (2006) Selectivity for multiple stimulus features in retinal ganglion cells. J Neurophysiol 96:2724-2738.

Famiglietti EV, Jr., Kolb H (1975) A bistratified amacrine cell and synaptic cirucitry in the inner plexiform layer of the retina. Brain Res 84:293-300.

Feigenspan A, Bormann J (1994) Differential pharmacology of GABAA and GABAC receptors on rat retinal bipolar cells. Eur J Pharmacol 288:97-104.

Feigenspan A, Bormann J (1998) GABA-gated Cl- channels in the rat retina. Prog Retin Eye Res 17:99-126.

Fischer F, Kneussel M, Tintrup H, Haverkamp S, Rauen T, Betz H, Wassle H (2000) Reduced synaptic clustering of GABA and glycine receptors in the retina of the gephyrin null mutant mouse. J Comp Neurol 427:634-648.

Fitzpatrick D, Lund JS, Schmechel DE, Towles AC (1987) Distribution of GABAergic neurons and axon terminals in the macaque striate cortex. J Comp Neurol 264:73-91.

Fletcher EL, Koulen P, Wassle H (1998) GABAA and GABAC receptors on mammalian rod bipolar cells. J Comp Neurol 396:351-365. 
Flores-Herr N, Protti DA, Wassle H (2001) Synaptic currents generating the inhibitory surround of ganglion cells in the mammalian retina. J Neurosci 21:4852-4863.

Frazao R, Nogueira MI, Wassle H (2007) Colocalization of synaptic GABA(C)-receptors with GABA (A)-receptors and glycine-receptors in the rodent central nervous system. Cell Tissue Res 330:1-15.

Freund TF (2003) Interneuron Diversity series: Rhythm and mood in perisomatic inhibition. Trends Neurosci 26:489-495.

Friedman HV, Bresler T, Garner CC, Ziv NE (2000) Assembly of new individual excitatory synapses: time course and temporal order of synaptic molecule recruitment. Neuron 27:57-69.

Fritschy JM, Mohler H (1995) GABAA-receptor heterogeneity in the adult rat brain: differential regional and cellular distribution of seven major subunits. J Comp Neurol 359:154-194.

Fritschy JM, Schweizer C, Brunig I, Luscher B (2003) Pre- and post-synaptic mechanisms regulating the clustering of type A gamma-aminobutyric acid receptors (GABAA receptors). Biochem Soc Trans 31:889-892.

Fu Z, Vicini S (2009) Neuroligin-2 accelerates GABAergic synapse maturation in cerebellar granule cells. Mol Cell Neurosci 42:45-55.

Fu Z, Washbourne P, Ortinski P, Vicini S (2003) Functional excitatory synapses in HEK293 cells expressing neuroligin and glutamate receptors. J Neurophysiol 90:3950-3957.

Garner CC, Zhai RG, Gundelfinger ED, Ziv NE (2002) Molecular mechanisms of CNS synaptogenesis. Trends Neurosci 25:243-251.

Gauthier J, Bonnel A, St-Onge J, Karemera L, Laurent S, Mottron L, Fombonne E, Joober R, Rouleau GA (2005) NLGN3/NLGN4 gene mutations are not responsible for autism in the Quebec population. Am J Med Genet B Neuropsychiatr Genet 132B:74-75.

Gibson JR, Huber KM, Sudhof TC (2009) Neuroligin-2 deletion selectively decreases inhibitory synaptic transmission originating from fast-spiking but not from somatostatin-positive interneurons. J Neurosci 29:13883-13897.

Gilbert MM, Auld VJ (2005) Evolution of clams (cholinesterase-like adhesion molecules): structure and function during development. Front Biosci 10:2177-2192.

Gill SB, Veruki ML, Hartveit E (2006) Functional properties of spontaneous IPSCs and glycine receptors in rod amacrine (All) cells in the rat retina. J Physiol 575:739-759.

Gillberg C (1998) Asperger syndrome and high-functioning autism. Br J Psychiatry 172:200-209.

Goodman CS (1996) Mechanisms and molecules that control growth cone guidance. Annu Rev Neurosci 19:341-377.

Grady EF, Baluk P, Bohm S, Gamp PD, Wong H, Payan DG, Ansel J, Portbury AL, Furness JB, McDonald DM, Bunnett NW (1996) Characterization of antisera specific to NK1, NK2, and NK3 neurokinin receptors and their utilization to localize receptors in the rat gastrointestinal tract. $J$ Neurosci 16:6975-6986.

Graf ER, Zhang X, Jin SX, Linhoff MW, Craig AM (2004) Neurexins induce differentiation of GABA and glutamate postsynaptic specializations via neuroligins. Cell 119:1013-1026.

Gray EG (1959) Electron microscopy of synaptic contacts on dendrite spines of the cerebral cortex. Nature 183:1592-1593.

Greferath U, Grunert U, Mohler H, Wassle H (1993a) Cholinergic amacrine cells of the rat retina express the delta-subunit of the GABAA-receptor. Neurosci Lett 163:71-73.

Greferath U, Muller F, Wassle H, Shivers B, Seeburg P (1993b) Localization of GABAA receptors in the rat retina. Vis Neurosci 10:551-561.

Greferath U, Grunert U, Fritschy JM, Stephenson A, Mohler H, Wassle H (1995) GABAA receptor subunits have differential distributions in the rat retina: in situ hybridization and immunohistochemistry. J Comp Neurol 353:553-571.

Grudzinska J, Schemm R, Haeger S, Nicke A, Schmalzing G, Betz H, Laube B (2005) The beta subunit determines the ligand binding properties of synaptic glycine receptors. Neuron 45:727739.

Grunert U (2000) Distribution of GABA and glycine receptors on bipolar and ganglion cells in the mammalian retina. Microsc Res Tech 50:130-140.

Grunert U, Wassle H (1993) Immunocytochemical localization of glycine receptors in the mammalian retina. J Comp Neurol 335:523-537.

Gunther U, Benson J, Benke D, Fritschy JM, Reyes G, Knoflach F, Crestani F, Aguzzi A, Arigoni M, Lang Y, et al. (1995) Benzodiazepine-insensitive mice generated by targeted disruption of the gamma 2 subunit gene of gamma-aminobutyric acid type A receptors. Proc Natl Acad Sci U S A 92:7749-7753. 
Gutierrez RC, Hung J, Zhang Y, Kertesz AC, Espina FJ, Colicos MA (2009) Altered synchrony and connectivity in neuronal networks expressing an autism-related mutation of neuroligin 3 . Neuroscience 162:208-221.

Hack I, Peichl L, Brandstatter JH (1999) An alternative pathway for rod signals in the rodent retina: rod photoreceptors, cone bipolar cells, and the localization of glutamate receptors. Proc Natl Acad Sci U S A 96:14130-14135.

Hack I, Frech M, Dick O, Peichl L, Brandstatter JH (2001) Heterogeneous distribution of AMPA glutamate receptor subunits at the photoreceptor synapses of rodent retina. Eur J Neurosci 13:15-24.

Hanley JG, Koulen P, Bedford F, Gordon-Weeks PR, Moss SJ (1999) The protein MAP-1B links $\mathrm{GABA}(\mathrm{C})$ receptors to the cytoskeleton at retinal synapses. Nature 397:66-69.

Hargrave PA, McDowell JH (1992) Rhodopsin and phototransduction: a model system for G proteinlinked receptors. Faseb J 6:2323-2331.

Harvey RJ, Schmieden V, Von Holst A, Laube B, Rohrer H, Betz H (2000) Glycine receptors containing the alpha4 subunit in the embryonic sympathetic nervous system, spinal cord and male genital ridge. Eur J Neurosci 12:994-1001.

Harvey RJ, Depner UB, Wassle H, Ahmadi S, Heindl C, Reinold H, Smart TG, Harvey K, Schutz B, Abo-Salem OM, Zimmer A, Poisbeau P, Welzl H, Wolfer DP, Betz H, Zeilhofer HU, Muller U (2004) GlyR alpha3: an essential target for spinal PGE2-mediated inflammatory pain sensitization. Science 304:884-887.

Haverkamp S, Wassle H (2000) Immunocytochemical analysis of the mouse retina. J Comp Neurol 424:1-23.

Haverkamp S, Grunert U, Wassle H (2000) The cone pedicle, a complex synapse in the retina. Neuron 27:85-95.

Haverkamp S, Grunert U, Wassle H (2001) The synaptic architecture of AMPA receptors at the cone pedicle of the primate retina. J Neurosci 21:2488-2500.

Haverkamp S, Muller U, Zeilhofer HU, Harvey RJ, Wassle H (2004) Diversity of glycine receptors in the mouse retina: localization of the alpha2 subunit. J Comp Neurol 477:399-411.

Haverkamp S, Muller U, Harvey K, Harvey RJ, Betz H, Wassle H (2003) Diversity of glycine receptors in the mouse retina: localization of the alpha3 subunit. J Comp Neurol 465:524-539.

Heidelberger R, Thoreson WB, Witkovsky P (2005) Synaptic transmission at retinal ribbon synapses. Prog Retin Eye Res 24:682-720.

Heidelberger R, Heinemann C, Neher E, Matthews G (1994) Calcium dependence of the rate of exocytosis in a synaptic terminal. Nature 371:513-515.

Heinze L, Harvey RJ, Haverkamp S, Wassle H (2007) Diversity of glycine receptors in the mouse retina: localization of the alpha4 subunit. J Comp Neurol 500:693-707.

Hirasawa $\mathrm{H}$, Kaneko A (2003) $\mathrm{pH}$ changes in the invaginating synaptic cleft mediate feedback from horizontal cells to cone photoreceptors by modulating Ca2+ channels. J Gen Physiol 122:657671.

Hua JY, Smith SJ (2004) Neural activity and the dynamics of central nervous system development. Nat Neurosci 7:327-332.

Hunter JW, Mullen GP, McManus JR, Heatherly JM, Duke A, Rand JB (2010) Neuroligin-deficient mutants of $C$. elegans have sensory processing deficits and are hypersensitive to oxidative stress and mercury toxicity. Dis Model Mech.

lacoboni M, Molnar-Szakacs I, Gallese V, Buccino G, Mazziotta JC, Rizzolatti G (2005) Grasping the intentions of others with one's own mirror neuron system. PLoS Biol 3:e79.

Ichtchenko K, Nguyen T, Sudhof TC (1996) Structures, alternative splicing, and neurexin binding of multiple neuroligins. J Biol Chem 271:2676-2682.

Ichtchenko K, Hata Y, Nguyen T, Ullrich B, Missler M, Moomaw C, Sudhof TC (1995) Neuroligin 1: a splice site-specific ligand for beta-neurexins. Cell 81:435-443.

Irie M, Hata Y, Takeuchi M, Ichtchenko K, Toyoda A, Hirao K, Takai Y, Rosahl TW, Sudhof TC (1997) Binding of neuroligins to PSD-95. Science 277:1511-1515.

Ivanova E, Muller U, Wassle H (2006) Characterization of the glycinergic input to bipolar cells of the mouse retina. Eur J Neurosci 23:350-364.

Jamain S, Quach H, Betancur C, Rastam M, Colineaux C, Gillberg IC, Soderstrom H, Giros B, Leboyer M, Gillberg C, Bourgeron T (2003) Mutations of the X-linked genes encoding neuroligins NLGN3 and NLGN4 are associated with autism. Nat Genet 34:27-29.

Jamain S, Radyushkin K, Hammerschmidt K, Granon S, Boretius S, Varoqueaux F, Ramanantsoa N, Gallego J, Ronnenberg A, Winter D, Frahm J, Fischer J, Bourgeron T, Ehrenreich H, Brose N 
(2008) Reduced social interaction and ultrasonic communication in a mouse model of monogenic heritable autism. Proc Natl Acad Sci U S A 105:1710-1715.

Jeon CJ, Strettoi E, Masland RH (1998) The major cell populations of the mouse retina. J Neurosci 18:8936-8946.

Johnson J, Tian N, Caywood MS, Reimer RJ, Edwards RH, Copenhagen DR (2003) Vesicular neurotransmitter transporter expression in developing postnatal rodent retina: GABA and glycine precede glutamate. J Neurosci 23:518-529.

Jung SY, Kim J, Kwon OB, Jung JH, An K, Jeong AY, Lee CJ, Choi YB, Bailey CH, Kandel ER, Kim JH (2010) Input-specific synaptic plasticity in the amygdala is regulated by neuroligin-1 via postsynaptic NMDA receptors. Proc Natl Acad Sci U S A.

Kamermans M, Fahrenfort I, Schultz K, Janssen-Bienhold U, Sjoerdsma T, Weiler R (2001) Hemichannel-mediated inhibition in the outer retina. Science 292:1178-1180.

Kemper TL, Bauman ML (2002) Neuropathology of infantile autism. Mol Psychiatry 7 Suppl 2:S12-13.

Kerschensteiner D, Wong RO (2008) A precisely timed asynchronous pattern of ON and OFF retinal ganglion cell activity during propagation of retinal waves. Neuron 58:851-858.

Kim E, Sheng M (2004) PDZ domain proteins of synapses. Nat Rev Neurosci 5:771-781.

Kim E, Niethammer M, Rothschild A, Jan YN, Sheng M (1995) Clustering of Shaker-type K+ channels by interaction with a family of membrane-associated guanylate kinases. Nature 378:85-88.

Kim HG, Kishikawa S, Higgins AW, Seong IS, Donovan DJ, Shen Y, Lally E, Weiss LA, Najm J, Kutsche K, Descartes M, Holt L, Braddock S, Troxell R, Kaplan L, Volkmar F, Klin A, Tsatsanis K, Harris DJ, Noens I, Pauls DL, Daly MJ, MacDonald ME, Morton CC, Quade BJ, Gusella JF (2008) Disruption of neurexin 1 associated with autism spectrum disorder. Am J Hum Genet 82:199-207.

Kins S, Betz H, Kirsch J (2000) Collybistin, a newly identified brain-specific GEF, induces submembrane clustering of gephyrin. Nat Neurosci 3:22-29.

Kirsch J (2006) Glycinergic transmission. Cell Tissue Res 326:535-540.

Kirsch J, Betz H (1993) Widespread expression of gephyrin, a putative glycine receptor-tubulin linker protein, in rat brain. Brain Res 621:301-310.

Kirsch J, Langosch D, Prior P, Littauer UZ, Schmitt B, Betz H (1991) The 93-kDa glycine receptorassociated protein binds to tubulin. J Biol Chem 266:22242-22245.

Kneussel M, Betz H (2000) Receptors, gephyrin and gephyrin-associated proteins: novel insights into the assembly of inhibitory postsynaptic membrane specializations. J Physiol $525 \mathrm{Pt}$ 1:1-9.

Kneussel M, Brandstatter JH, Laube B, Stahl S, Muller U, Betz H (1999) Loss of postsynaptic $\mathrm{GABA}(\mathrm{A})$ receptor clustering in gephyrin-deficient mice. J Neurosci 19:9289-9297.

Knott GW, Quairiaux C, Genoud C, Welker E (2002) Formation of dendritic spines with GABAergic synapses induced by whisker stimulation in adult mice. Neuron 34:265-273.

Kolb H (2006) Funtional Organization of the retina in 'Principles and Practice of Clinical Electrophysiology of Vision' (2nd edn) edited by John R. Heckenlively and Geoffrey B. Arden: MIT press.

Kornau HC, Schenker LT, Kennedy MB, Seeburg PH (1995) Domain interaction between NMDA receptor subunits and the postsynaptic density protein PSD-95. Science 269:1737-1740.

Kotak VC, Korada S, Schwartz IR, Sanes DH (1998) A developmental shift from GABAergic to glycinergic transmission in the central auditory system. J Neurosci 18:4646-4655.

Koulen P (1997) Vesicular acetylcholine transporter (VAChT): a cellular marker in rat retinal development. Neuroreport 8:2845-2848.

Koulen P, Sassoe-Pognetto M, Grunert U, Wassle H (1996) Selective clustering of GABA(A) and glycine receptors in the mammalian retina. J Neurosci 16:2127-2140.

Koulen P, Brandstatter JH, Enz R, Bormann J, Wassle H (1998a) Synaptic clustering of GABA(C) receptor rho-subunits in the rat retina. Eur $\mathrm{J}$ Neurosci 10:115-127.

Koulen P, Fletcher EL, Craven SE, Bredt DS, Wassle H (1998b) Immunocytochemical localization of the postsynaptic density protein PSD-95 in the mammalian retina. J Neurosci 18:10136-10149.

Koulen P, Brandstatter JH, Kroger S, Enz R, Bormann J, Wassle H (1997) Immunocytochemical localization of the $\mathrm{GABA}(\mathrm{C})$ receptor rho subunits in the cat, goldfish, and chicken retina. J Comp Neurol 380:520-532.

Koulen P, Malitschek B, Kuhn R, Bettler B, Wassle H, Brandstatter JH (1998c) Presynaptic and postsynaptic localization of $\operatorname{GABA}(\mathrm{B})$ receptors in neurons of the rat retina. Eur $\mathrm{J}$ Neurosci 10:1446-1456.

Landis DM (1988) Membrane and cytoplasmic structure at synaptic junctions in the mammalian central nervous system. J Electron Microsc Tech 10:129-151. 
Langosch D, Becker CM, Betz H (1990) The inhibitory glycine receptor: a ligand-gated chloride channel of the central nervous system. Eur J Biochem 194:1-8.

Lardi-Studler B, Fritschy JM (2007) Matching of pre- and postsynaptic specializations during synaptogenesis. Neuroscientist 13:115-126.

Laumonnier F, Bonnet-Brilhault F, Gomot M, Blanc R, David A, Moizard MP, Raynaud M, Ronce N, Lemonnier E, Calvas P, Laudier B, Chelly J, Fryns JP, Ropers HH, Hamel BC, Andres C, Barthelemy C, Moraine C, Briault S (2004) X-linked mental retardation and autism are associated with a mutation in the NLGN4 gene, a member of the neuroligin family. Am J Hum Genet 74:552-557.

Legendre $P$ (2001) The glycinergic inhibitory synapse. Cell Mol Life Sci 58:760-793.

Levinson JN, Chery N, Huang K, Wong TP, Gerrow K, Kang R, Prange O, Wang YT, El-Husseini A (2005) Neuroligins mediate excitatory and inhibitory synapse formation: involvement of PSD95 and neurexin-1beta in neuroligin-induced synaptic specificity. J Biol Chem 280:1731217319.

Levitt P, Eagleson KL, Powell EM (2004) Regulation of neocortical interneuron development and the implications for neurodevelopmental disorders. Trends Neurosci 27:400-406.

Linhoff MW, Lauren J, Cassidy RM, Dobie FA, Takahashi H, Nygaard HB, Airaksinen MS, Strittmatter SM, Craig AM (2009) An unbiased expression screen for synaptogenic proteins identifies the LRRTM protein family as synaptic organizers. Neuron 61:734-749.

Lise MF, El-Husseini A (2006) The neuroligin and neurexin families: from structure to function at the synapse. Cell Mol Life Sci 63:1833-1849.

Liu Q, Wong-Riley MT (2005) Postnatal developmental expressions of neurotransmitters and receptors in various brain stem nuclei of rats. J Appl Physiol 98:1442-1457.

Lopez-Corcuera B, Geerlings A, Aragon C (2001) Glycine neurotransmitter transporters: an update. Mol Membr Biol 18:13-20.

Lorez M, Benke D, Luscher B, Mohler H, Benson JA (2000) Single-channel properties of neuronal GABAA receptors from mice lacking the 2 subunit. J Physiol 527 Pt 1:11-31.

Lui L, Levinson JN, Noel G, Handrigan GR, Richman JM, El-Husseini A, Moukhles H (2010) Synaptic localization of neuroligin 2 in the rodent retina: comparative study with the dystroglycancontaining complex. J Neurosci Res 88:837-849.

Lukasiewicz PD (1996) GABAC receptors in the vertebrate retina. Mol Neurobiol 12:181-194.

Lukasiewicz PD, Shields CR (1998) A diversity of GABA receptors in the retina. Semin Cell Dev Biol 9:293-299.

Lukasiewicz PD, Eggers ED, Sagdullaev BT, McCall MA (2004) GABAC receptor-mediated inhibition in the retina. Vision Res 44:3289-3296.

Ma DQ, Whitehead PL, Menold MM, Martin ER, Ashley-Koch AE, Mei H, Ritchie MD, Delong GR, Abramson RK, Wright HH, Cuccaro ML, Hussman JP, Gilbert JR, Pericak-Vance MA (2005) Identification of significant association and gene-gene interaction of GABA receptor subunit genes in autism. Am J Hum Genet 77:377-388.

Macdonald RL, Olsen RW (1994) GABAA receptor channels. Annu Rev Neurosci 17:569-602.

MacNeil MA, Masland RH (1998) Extreme diversity among amacrine cells: implications for function. Neuron 20:971-982.

Majumdar S, Weiss J, Wassle H (2009) Glycinergic input of widefield, displaced amacrine cells of the mouse retina. J Physiol 587:3831-3849.

Majumdar S, Heinze L, Haverkamp S, Ivanova E, Wassle H (2007) Glycine receptors of A-type ganglion cells of the mouse retina. Vis Neurosci 24:471-487.

Mandell JW, Czernik AJ, De Camilli P, Greengard P, Townes-Anderson E (1992) Differential expression of synapsins I and II among rat retinal synapses. J Neurosci 12:1736-1749.

Mann EO, Paulsen O (2007) Role of GABAergic inhibition in hippocampal network oscillations. Trends Neurosci 30:343-349.

Marc RE (1989) The role of glycine in the mammalian retina. Prog Retinal Res 8:67-107.

Markram H, Toledo-Rodriguez M, Wang Y, Gupta A, Silberberg G, Wu C (2004) Interneurons of the neocortical inhibitory system. Nat Rev Neurosci 5:793-807.

Masland RH (2001a) The fundamental plan of the retina. Nat Neurosci 4:877-886.

Masland RH (2001b) Neuronal diversity in the retina. Curr Opin Neurobiol 11:431-436.

Matsubara A, Laake JH, Davanger S, Usami S, Ottersen OP (1996) Organization of AMPA receptor subunits at a glutamate synapse: a quantitative immunogold analysis of hair cell synapses in the rat organ of Corti. J Neurosci 16:4457-4467.

Maximov A, Sudhof TC, Bezprozvanny I (1999) Association of neuronal calcium channels with modular adaptor proteins. J Biol Chem 274:24453-24456. 
McBain CJ, Fisahn A (2001) Interneurons unbound. Nat Rev Neurosci 2:11-23.

McCall MA, Lukasiewicz PD, Gregg RG, Peachey NS (2002) Elimination of the rho1 subunit abolishes $\mathrm{GABA}(\mathrm{C})$ receptor expression and alters visual processing in the mouse retina. J Neurosci 22:4163-4174.

Menger N, Pow DV, Wassle H (1998) Glycinergic amacrine cells of the rat retina. J Comp Neurol 401:34-46.

Meyer G, Kirsch J, Betz H, Langosch D (1995) Identification of a gephyrin binding motif on the glycine receptor beta subunit. Neuron 15:563-572.

Meyer G, Varoqueaux F, Neeb A, Oschlies M, Brose N (2004) The complexity of PDZ domainmediated interactions at glutamatergic synapses: a case study on neuroligin. Neuropharmacology 47:724-733.

Misgeld U, Bijak M, Jarolimek W (1995) A physiological role for GABAB receptors and the effects of baclofen in the mammalian central nervous system. Prog Neurobiol 46:423-462.

Missler M, Sudhof TC (1998) Neurexins: three genes and 1001 products. Trends Genet 14:20-26.

Missler M, Zhang W, Rohlmann A, Kattenstroth G, Hammer RE, Gottmann K, Sudhof TC (2003) Alpha-neurexins couple Ca2+ channels to synaptic vesicle exocytosis. Nature 423:939-948.

Mody I, De Koninck Y, Otis TS, Soltesz I (1994) Bridging the cleft at GABA synapses in the brain. Trends Neurosci 17:517-525.

Montero VM, Zempel J (1986) The proportion and size of GABA-immunoreactive neurons in the magnocellular and parvocellular layers of the lateral geniculate nucleus of the rhesus monkey. Exp Brain Res 62:215-223.

Morgans CW (2000) Neurotransmitter release at ribbon synapses in the retina. Immunol Cell Biol $78: 442-446$.

Moss SJ, Smart TG (2001) Constructing inhibitory synapses. Nat Rev Neurosci 2:240-250.

Mumm JS, Godinho L, Morgan JL, Oakley DM, Schroeter EH, Wong RO (2005) Laminar circuit formation in the vertebrate retina. Prog Brain Res 147:155-169.

Murphy GJ, Rieke F (2008) Signals and noise in an inhibitory interneuron diverge to control activity in nearby retinal ganglion cells. Nat Neurosci 11:318-326.

Nirenberg S, Meister M (1997) The light response of retinal ganglion cells is truncated by a displaced amacrine circuit. Neuron 18:637-650.

Nomura A, Shigemoto R, Nakamura Y, Okamoto N, Mizuno N, Nakanishi S (1994) Developmentally regulated postsynaptic localization of a metabotropic glutamate receptor in rat rod bipolar cells. Cell 77:361-369.

O'Brien BJ, Richardson RC, Berson DM (2003) Inhibitory network properties shaping the light evoked responses of cat alpha retinal ganglion cells. Vis Neurosci 20:351-361.

O'Brien JA, Berger AJ (1999) Cotransmission of GABA and glycine to brain stem motoneurons. J Neurophysiol 82:1638-1641.

O'Sullivan GA, Hofer W, Betz H (2009) Inhibitory postsynaptic membrane specializations are formed in gephyrin-deficient mice. Neurosci Lett 458:106-110.

Okabe S (2007) Molecular anatomy of the postsynaptic density. Mol Cell Neurosci 34:503-518.

Okabe S, Miwa A, Okado H (2001) Spine formation and correlated assembly of presynaptic and postsynaptic molecules. J Neurosci 21:6105-6114.

Panzanelli P, Homanics GE, Ottersen OP, Fritschy JM, Sassoe-Pognetto M (2004) Pre- and postsynaptic GABA receptors at reciprocal dendrodendritic synapses in the olfactory bulb. Eur J Neurosci 20:2945-2952.

Papadopoulos T, Korte M, Eulenburg V, Kubota H, Retiounskaia M, Harvey RJ, Harvey K, O'Sullivan GA, Laube B, Hulsmann S, Geiger JR, Betz H (2007) Impaired GABAergic transmission and altered hippocampal synaptic plasticity in collybistin-deficient mice. Embo J 26:3888-3899.

Patrizi A, Scelfo B, Viltono L, Briatore F, Fukaya M, Watanabe M, Strata P, Varoqueaux F, Brose N, Fritschy JM, Sassoe-Pognetto M (2008) Synapse formation and clustering of neuroligin-2 in the absence of GABAA receptors. Proc Natl Acad Sci U S A 105:13151-13156.

Pattnaik B, Jellali A, Sahel J, Dreyfus H, Picaud S (2000) GABAC receptors are localized with microtubule-associated protein 1B in mammalian cone photoreceptors. J Neurosci 20:67896796.

Peichl L, Gonzalez-Soriano J (1994) Morphological types of horizontal cell in rodent retinae: a comparison of rat, mouse, gerbil, and guinea pig. Vis Neurosci 11:501-517.

Persico AM, Bourgeron T (2006) Searching for ways out of the autism maze: genetic, epigenetic and environmental clues. Trends Neurosci 29:349-358.

Peters A, Palay SL, Webster HD (1991) The Fine Structure of the Nervous System: Neurons and Their Supporting Cells (3rd edn). New York: Oxford University press. 
Pierce JP, Mendell LM (1993) Quantitative ultrastructure of la boutons in the ventral horn: scaling and positional relationships. J Neurosci 13:4748-4763.

Poulopoulos A, Aramuni G, Meyer G, Soykan T, Hoon M, Papadopoulos T, Zhang M, Paarmann I, Fuchs C, Harvey K, Jedlicka P, Schwarzacher SW, Betz H, Harvey RJ, Brose N, Zhang W, Varoqueaux F (2009) Neuroligin 2 drives postsynaptic assembly at perisomatic inhibitory synapses through gephyrin and collybistin. Neuron 63:628-642.

Pourcho RG, Goebel DJ (1985) A combined Golgi and autoradiographic study of (3H)glycineaccumulating amacrine cells in the cat retina. J Comp Neurol 233:473-480.

Pourcho RG, Owczarzak MT (1991) Connectivity of glycine immunoreactive amacrine cells in the cat retina. J Comp Neurol 307:549-561.

Pow DV, Hendrickson AE (1999) Distribution of the glycine transporter glyt-1 in mammalian and nonmammalian retinae. Vis Neurosci 16:231-239.

Prange O, Wong TP, Gerrow K, Wang YT, El-Husseini A (2004) A balance between excitatory and inhibitory synapses is controlled by PSD-95 and neuroligin. Proc Natl Acad Sci U S A 101:13915-13920.

Prior P, Schmitt B, Grenningloh G, Pribilla I, Multhaup G, Beyreuther K, Maulet Y, Werner P, Langosch D, Kirsch J, et al. (1992) Primary structure and alternative splice variants of gephyrin, a putative glycine receptor-tubulin linker protein. Neuron 8:1161-1170.

Protti DA, Gerschenfeld HM, Llano I (1997) GABAergic and glycinergic IPSCs in ganglion cells of rat retinal slices. J Neurosci 17:6075-6085.

Radyushkin K, Hammerschmidt K, Boretius S, Varoqueaux F, El-Kordi A, Ronnenberg A, Winter D, Frahm J, Fischer J, Brose N, Ehrenreich H (2009) Neuroligin-3-deficient mice: model of a monogenic heritable form of autism with an olfactory deficit. Genes Brain Behav 8:416-425.

Raviola E, Dacheux RF (1987) Excitatory dyad synapse in rabbit retina. Proc Natl Acad Sci U S A 84:7324-7328.

Reid T, Bathoorn A, Ahmadian MR, Collard JG (1999) Identification and characterization of hPEM-2, a guanine nucleotide exchange factor specific for Cdc42. J Biol Chem 274:33587-33593.

Ritvo ER, Creel D, Realmuto G, Crandall AS, Freeman BJ, Bateman JB, Barr R, Pingree C, Coleman M, Purple R (1988) Electroretinograms in autism: a pilot study of b-wave amplitudes. Am J Psychiatry 145:229-232.

Rothe T, Juttner R, Bahring R, Grantyn R (1999) Ion conductances related to development of repetitive firing in mouse retinal ganglion neurons in situ. J Neurobiol 38:191-206.

Rubenstein JL, Merzenich MM (2003) Model of autism: increased ratio of excitation/inhibition in key neural systems. Genes Brain Behav 2:255-267.

Rudenko G, Nguyen T, Chelliah Y, Sudhof TC, Deisenhofer J (1999) The structure of the ligandbinding domain of neurexin lbeta: regulation of LNS domain function by alternative splicing. Cell 99:93-101.

Sagne C, El Mestikawy S, Isambert MF, Hamon M, Henry JP, Giros B, Gasnier B (1997) Cloning of a functional vesicular GABA and glycine transporter by screening of genome databases. FEBS Lett 417:177-183.

Sakai HM, Naka K (1992) Response dynamics and receptive-field organization of catfish amacrine cells. J Neurophysiol 67:430-442.

Salinas E, Sejnowski TJ (2001) Correlated neuronal activity and the flow of neural information. Nat Rev Neurosci 2:539-550.

Sankaranarayanan S, Atluri PP, Ryan TA (2003) Actin has a molecular scaffolding, not propulsive, role in presynaptic function. Nat Neurosci 6:127-135.

Sassoe-Pognetto M, Wassle H (1997) Synaptogenesis in the rat retina: subcellular localization of glycine receptors, GABA(A) receptors, and the anchoring protein gephyrin. J Comp Neurol 381:158-174.

Sassoe-Pognetto M, Wassle H, Grunert U (1994) Glycinergic synapses in the rod pathway of the rat retina: cone bipolar cells express the alpha 1 subunit of the glycine receptor. J Neurosci 14:5131-5146.

Sassoe-Pognetto M, Kirsch J, Grunert U, Greferath U, Fritschy JM, Mohler H, Betz H, Wassle H (1995) Colocalization of gephyrin and GABAA-receptor subunits in the rat retina. J Comp Neurol 357:1-14.

Scheiffele P, Fan J, Choih J, Fetter R, Serafini T (2000) Neuroligin expressed in nonneuronal cells triggers presynaptic development in contacting axons. Cell 101:657-669.

Schikorski T, Stevens CF (1997) Quantitative ultrastructural analysis of hippocampal excitatory synapses. J Neurosci 17:5858-5867. 
Schmitz F, Konigstorfer A, Sudhof TC (2000) RIBEYE, a component of synaptic ribbons: a protein's journey through evolution provides insight into synaptic ribbon function. Neuron 28:857-872.

Schoch S, Gundelfinger ED (2006) Molecular organization of the presynaptic active zone. Cell Tissue Res 326:379-391.

Schweizer C, Balsiger S, Bluethmann H, Mansuy IM, Fritschy JM, Mohler H, Luscher B (2003) The gamma 2 subunit of $\operatorname{GABA}(\mathrm{A})$ receptors is required for maintenance of receptors at mature synapses. Mol Cell Neurosci 24:442-450.

Seeburg PH, Wisden W, Verdoorn TA, Pritchett DB, Werner P, Herb A, Luddens H, Sprengel R, Sakmann B (1990) The GABAA receptor family: molecular and functional diversity. Cold Spring Harb Symp Quant Biol 55:29-40.

Shatz CJ, Stryker MP (1978) Ocular dominance in layer IV of the cat's visual cortex and the effects of monocular deprivation. J Physiol 281:267-283.

Shen K (2004) Molecular mechanisms of target specificity during synapse formation. Curr Opin Neurobiol 14:83-88.

Sheng M, Hoogenraad CC (2007) The postsynaptic architecture of excitatory synapses: a more quantitative view. Annu Rev Biochem 76:823-847.

Sieghart W (1995) Structure and pharmacology of gamma-aminobutyric acidA receptor subtypes. Pharmacol Rev 47:181-234.

Sillito AM (1974) Proceedings: Modification of the receptive field properties of neurones in the visual cortex by bicuculline, a GABA antagonist. J Physiol 239:36P-37P.

Sillito AM, Kemp JA (1983) The influence of GABAergic inhibitory processes on the receptive field structure of $X$ and $Y$ cells in cat dorsal lateral geniculate nucleus (dLGN). Brain Res 277:6377.

Sillito AM, Salt TE, Kemp JA (1985) Modulatory and inhibitory processes in the visual cortex. Vision Res 25:375-381.

Sinclair JR, Jacobs AL, Nirenberg S (2004) Selective ablation of a class of amacrine cells alters spatial processing in the retina. J Neurosci 24:1459-1467.

Singer JH, Talley EM, Bayliss DA, Berger AJ (1998) Development of glycinergic synaptic transmission to rat brain stem motoneurons. J Neurophysiol 80:2608-2620.

Singer W (1999) Neuronal synchrony: a versatile code for the definition of relations? Neuron 24:49-65, 111-125.

Singer W, Gray CM (1995) Visual feature integration and the temporal correlation hypothesis. Annu Rev Neurosci 18:555-586.

Slaughter MM, Pan ZH (1992) The physiology of GABAB receptors in the vertebrate retina. Prog Brain Res 90:47-60.

Smith AJ, Owens S, Forsythe ID (2000) Characterisation of inhibitory and excitatory postsynaptic currents of the rat medial superior olive. J Physiol 529 Pt 3:681-698.

Song JY, Ichtchenko K, Sudhof TC, Brose N (1999) Neuroligin 1 is a postsynaptic cell-adhesion molecule of excitatory synapses. Proc Natl Acad Sci U S A 96:1100-1105.

Soucy E, Wang Y, Nirenberg S, Nathans J, Meister M (1998) A novel signaling pathway from rod photoreceptors to ganglion cells in mammalian retina. Neuron 21:481-493.

Sterling P, Matthews G (2005) Structure and function of ribbon synapses. Trends Neurosci 28:20-29.

Strettoi E, Dacheux RF, Raviola E (1990) Synaptic connections of rod bipolar cells in the inner plexiform layer of the rabbit retina. J Comp Neurol 295:449-466.

Strettoi E, Raviola E, Dacheux RF (1992) Synaptic connections of the narrow-field, bistratified rod amacrine cell (All) in the rabbit retina. J Comp Neurol 325:152-168.

Stryer L (1991) Visual excitation and recovery. J Biol Chem 266:10711-10714.

Sudhof TC (2004) The synaptic vesicle cycle. Annu Rev Neurosci 27:509-547.

Sudhof TC (2008) Neuroligins and neurexins link synaptic function to cognitive disease. Nature 455:903-911.

Suzuki H, Pinto LH (1986) Response properties of horizontal cells in the isolated retina of wild-type and pearl mutant mice. J Neurosci 6:1122-1128.

Szel A, Rohlich P, Caffe AR, Juliusson B, Aguirre G, Van Veen T (1992) Unique topographic separation of two spectral classes of cones in the mouse retina. J Comp Neurol 325:327-342.

Tabuchi K, Sudhof TC (2002) Structure and evolution of neurexin genes: insight into the mechanism of alternative splicing. Genomics 79:849-859.

Tabuchi K, Blundell J, Etherton MR, Hammer RE, Liu X, Powell CM, Sudhof TC (2007) A neuroligin-3 mutation implicated in autism increases inhibitory synaptic transmission in mice. Science 318:71-76.

Takamori S (2006) VGLUTs: 'exciting' times for glutamatergic research? Neurosci Res 55:343-351. 
Tian N, Hwang TN, Copenhagen DR (1998) Analysis of excitatory and inhibitory spontaneous synaptic activity in mouse retinal ganglion cells. J Neurophysiol 80:1327-1340.

tom Dieck S, Brandstatter JH (2006) Ribbon synapses of the retina. Cell Tissue Res 326:339-346.

Tsukamoto Y, Morigiwa K, Ueda M, Sterling P (2001) Microcircuits for night vision in mouse retina. J Neurosci 21:8616-8623.

Ullrich B, Ushkaryov YA, Sudhof TC (1995) Cartography of neurexins: more than 1000 isoforms generated by alternative splicing and expressed in distinct subsets of neurons. Neuron 14:497507.

Vaney DI (1990) The mosaic of amacrine cells in the mammalian

retina. Prog Retinal Res 9:49-100.

Vardi N (1998) Alpha subunit of Go localizes in the dendritic tips of ON bipolar cells. J Comp Neurol 395:43-52.

Vardi N, Auerbach P (1995) Specific cell types in cat retina express different forms of glutamic acid decarboxylase. J Comp Neurol 351:374-384.

Vardi N, Kaufman DL, Sterling P (1994) Horizontal cells in cat and monkey retina express different isoforms of glutamic acid decarboxylase. Vis Neurosci 11:135-142.

Vardi N, Duvoisin R, Wu G, Sterling P (2000) Localization of mGluR6 to dendrites of ON bipolar cells in primate retina. J Comp Neurol 423:402-412.

Varoqueaux F, Jamain S, Brose N (2004) Neuroligin 2 is exclusively localized to inhibitory synapses. Eur J Cell Biol 83:449-456.

Varoqueaux F, Sigler A, Rhee JS, Brose N, Enk C, Reim K, Rosenmund C (2002) Total arrest of spontaneous and evoked synaptic transmission but normal synaptogenesis in the absence of Munc13-mediated vesicle priming. Proc Natl Acad Sci U S A 99:9037-9042.

Varoqueaux F, Aramuni G, Rawson RL, Mohrmann R, Missler M, Gottmann K, Zhang W, Sudhof TC, Brose N (2006) Neuroligins determine synapse maturation and function. Neuron 51:741-754.

Verhage M, Maia AS, Plomp JJ, Brussaard AB, Heeroma JH, Vermeer H, Toonen RF, Hammer RE, van den Berg TK, Missler M, Geuze HJ, Sudhof TC (2000) Synaptic assembly of the brain in the absence of neurotransmitter secretion. Science 287:864-869.

Vincent JB, Kolozsvari D, Roberts WS, Bolton PF, Gurling HM, Scherer SW (2004) Mutation screening of X-chromosomal neuroligin genes: no mutations in 196 autism probands. Am J Med Genet B Neuropsychiatr Genet 129B:82-84.

von Gersdorff H, Vardi E, Matthews G, Sterling P (1996) Evidence that vesicles on the synaptic ribbon of retinal bipolar neurons can be rapidly released. Neuron 16:1221-1227.

Wachtmeister $L$ (1980) Further studies of the chemical sensitivity of the oscillatory potentials of the electroretinogram (ERG) I. GABA- and glycine antagonists. Acta Ophthalmol (Copenh) 58:712-725.

Wachtmeister L (1998) Oscillatory potentials in the retina: what do they reveal. Prog Retin Eye Res 17:485-521.

Wachtmeister L, Dowling JE (1978) The oscillatory potentials of the mudpuppy retina. Invest Ophthalmol Vis Sci 17:1176-1188.

Waites CL, Craig AM, Garner CC (2005) Mechanisms of vertebrate synaptogenesis. Annu Rev Neurosci 28:251-274.

Wang CT, Blankenship AG, Anishchenko A, Elstrott J, Fikhman M, Nakanishi S, Feller MB (2007) GABA(A) receptor-mediated signaling alters the structure of spontaneous activity in the developing retina. J Neurosci 27:9130-9140.

Washbourne P, Dityatev A, Scheiffele P, Biederer T, Weiner JA, Christopherson KS, El-Husseini A (2004) Cell adhesion molecules in synapse formation. J Neurosci 24:9244-9249.

Wassle H (2004) Parallel processing in the mammalian retina. Nat Rev Neurosci 5:747-757.

Wassle H, Boycott BB (1991) Functional architecture of the mammalian retina. Physiol Rev 71:447480.

Wassle H, Schafer-Trenkler I, Voigt T (1986) Analysis of a glycinergic inhibitory pathway in the cat retina. J Neurosci 6:594-604.

Wassle H, Koulen P, Brandstatter JH, Fletcher EL, Becker CM (1998) Glycine and GABA receptors in the mammalian retina. Vision Res 38:1411-1430.

Wassle H, Heinze L, Ivanova E, Majumdar S, Weiss J, Harvey RJ, Haverkamp S (2009) Glycinergic transmission in the Mammalian retina. Front Mol Neurosci 2:6.

Weiss J, O'Sullivan GA, Heinze L, Chen HX, Betz H, Wassle H (2008) Glycinergic input of small-field amacrine cells in the retinas of wildtype and glycine receptor deficient mice. Mol Cell Neurosci $37: 40-55$.

Witkovsky P (2004) Dopamine and retinal function. Doc Ophthalmol 108:17-40. 
Wojcik SM, Katsurabayashi S, Guillemin I, Friauf E, Rosenmund C, Brose N, Rhee JS (2006) A shared vesicular carrier allows synaptic corelease of GABA and glycine. Neuron 50:575-587.

Wong RO (1999) Retinal waves and visual system development. Annu Rev Neurosci 22:29-47.

Wong WT, Wong RO (2001) Changing specificity of neurotransmitter regulation of rapid dendritic remodeling during synaptogenesis. Nat Neurosci 4:351-352.

Yamagata M, Sanes JR, Weiner JA (2003) Synaptic adhesion molecules. Curr Opin Cell Biol 15:621632.

Yan J, Oliveira G, Coutinho A, Yang C, Feng J, Katz C, Sram J, Bockholt A, Jones IR, Craddock N, Cook EH, Jr., Vicente A, Sommer SS (2005) Analysis of the neuroligin 3 and 4 genes in autism and other neuropsychiatric patients. Mol Psychiatry 10:329-332.

Zafra F, Aragon C, Gimenez C (1997) Molecular biology of glycinergic neurotransmission. Mol Neurobiol 14:117-142.

Zafra F, Aragon C, Olivares L, Danbolt NC, Gimenez C, Storm-Mathisen J (1995) Glycine transporters are differentially expressed among CNS cells. J Neurosci 15:3952-3969.

Zhai RG, Vardinon-Friedman H, Cases-Langhoff C, Becker B, Gundelfinger ED, Ziv NE, Garner CC (2001) Assembling the presynaptic active zone: a characterization of an active one precursor vesicle. Neuron 29:131-143.

Zhang C, Milunsky JM, Newton S, Ko J, Zhao G, Maher TA, Tager-Flusberg H, Bolliger MF, Carter AS, Boucard AA, Powell CM, Sudhof TC (2009) A neuroligin-4 missense mutation associated with autism impairs neuroligin-4 folding and endoplasmic reticulum export. J Neurosci 29:1084310854.

Zhang J, Jung CS, Slaughter MM (1997) Serial inhibitory synapses in retina. Vis Neurosci 14:553-563.

Zhang J, Yang Z, Wu SM (2005) Development of cholinergic amacrine cells is visual activitydependent in the postnatal mouse retina. J Comp Neurol 484:331-343.

Zoghbi HY (2003) Postnatal neurodevelopmental disorders: meeting at the synapse? Science 302:826-830. 


\section{Curriculum Vitae}

\section{PERSONAL \\ INFORMATION}

$\begin{array}{lcl}\text { ADDRESS } & \vdots & \text { Mrinalini Hoon } \\ & \vdots & \text { Department of Molecular Neurobiology } \\ & \vdots & \text { Max-Planck Institute for Experimental Medicine } \\ & \vdots & \\ & \vdots & \\ & \vdots & \\ \text { EMAIL } & \vdots & \\ & & \\ \text { DATE OF BIRTH } & 12^{\text {th }} \text { September } 1984 \\ & \vdots & \\ \text { NATIONALITY } & \vdots & \\ & & \\ & & \end{array}$

\section{EDUCATION}

Oct 2006-2010

Max Planck Institute for Experimental Medicine, Goettingen

$\mathrm{PhD}$ at the Department of Molecular Neurobiology

Thesis: "Role of Neuroligins at the Inhibitory Postsynaptic Compartment of the Retina"

Under the supervision of Prof. Dr. Nils Brose and Dr. Frederique Varoqueaux

Oct 2006-2009

Neuroscience Early Stage Research Training program (NEUREST)

Sept 2005-2010

Georg-August-Universitat, Goettingen

International Max Planck Research School

MSc/PhD Neuroscience Program

2002-2005

Calcutta University

Presidency College

BSc (Hons) in Physiology 


\section{PROJECTS \& RESEARCH EXPERIENCE}

2006

2006

2006

2006

2005

2004
Max Planck Institute for Experimental Medicine, Goettingen

Department of Molecular Neurobiology

"Morphological study of Neuroligin 1 and 2 knockout mice"

German Primate Center, Goettingen

Department of Neurobiology

"Localization of stress regulated glycoprotein M6a in the hippocampus"

Max Planck Institute for Biophysical Chemistry, Goettingen

Department of Biomedical NMR Research

"fMRI Studies of the Human Somatosensory Cortex"

Georg-August University, Goettingen

Center for Physiology and Pathophysiology

"EGFP tagging of $\alpha$-subunit of the $\mathrm{N}$-type calcium channel"

Jawaharlal Nehru Centre for Advanced Scientific Research, India

"Molecular Genetic Study of Non-Syndromic Hearing Loss (NSHL) related to CDH23"

University of Calcutta, Department of Physiology

Survey and analysis of social and physiological conditions (cardiovascular haematological and anthropometric parameters) in isolated tribal habitants

\section{SUMMER SCHOOL TRAINING}

2008

European Synapse Summer School

PENS training center

Bordeaux, France 


\title{
TEACHING EXPERIENCE
}

2009

2009

Labrotation Supervisor for Ms. Helena de Fatima Magliarelli

International MSc/PhD Molecular Biology Program

Tutor for the lectures "Nervous System 1.1" and "Nervous System 1.2"

International MSc/PhD Molecular Biology Program

\author{
AWARDS \& SCHOLARSHIPS \\ 2006-2009 \\ NEUREST: Marie Curie PhD Fellowship \\ Stipend, International Max Planck Research School, Germany \\ Summer Research fellowship at Jawaharlal Nehru Centre for Advanced \\ Scientific Research, India \\ 2004-2005 \\ Award of merit for BSc (Hons) Calcutta University Examinations
}

\section{CONFERENCES}

2009

Society for Neuroscience meeting, Chicago, USA

Poster presentation

2009

European Retina meeting, Oldenburg, Germany

Poster presentation

2009

$8^{\text {th }}$ meeting of the German Neuroscience Society, Goettingen, Germany

Poster presentation

2008

Federation of European Neurosciences (FENS) meeting, Geneva, Switzerland Poster presentation

European Retina meeting, Frankfurt, Germany

Poster Presentation 
EXTRA-CURRICULAR ACTIVITIES

2009

Co-organizer of NEURIZONS 2009 meeting, held at the Max Planck Institute for Experimental medicine $\left(13^{\text {th }}-16^{\text {th }}\right.$ May 2009), Goettingen, Germany 


\section{Publication list}

Hoon M, Bauer G, Fritschy JM, Moser T, Falkenburger BH, Varoqueaux F (2009) Neuroligin 2 controls the maturation of GABAergic synapses and information processing in the retina. $J$. Neuroscience. 29: 8039-8050.

Poulopoulos A, Aramuni G, Meyer G, Soykan T, Hoon M, Papadopoulos T, Zhang M, Paarmann I, Fuchs C, Harvey K, Jedlicka P, Schwarzacher SW, Betz H, Harvey RJ, Brose N, Zhang W, Varoqueaux F (2009)

Neuroligin 2 drives postsynaptic assembly at perisomatic inhibitory synapses through gephyrin and collybistin. Neuron. 2009 Sep 10; 63(5): 628-42.

Jedlicka P, Hoon M, Papadopoulos T, Vlachos A, Winkels R, Poulopoulos A, Betz H, Deller T, Brose N, Varoqueaux F, Schwarzacher S. W Increased Dentate Gyrus Excitability in Neuroligin 2-Deficient Mice in Vivo. Cerebral Cortex (accepted)

Hoon M, Falkenburger B, Soykan T, Hammer M, Patrizi A, Schmidt K.F, Sassoe-Pognetto M, Loewel S, Moser T, Taschenberger H, Brose N, Varoqueaux F.

Neuroligin-4 is localized to glycinergic postsynapses and regulates inhibition in the retina (submitted) 